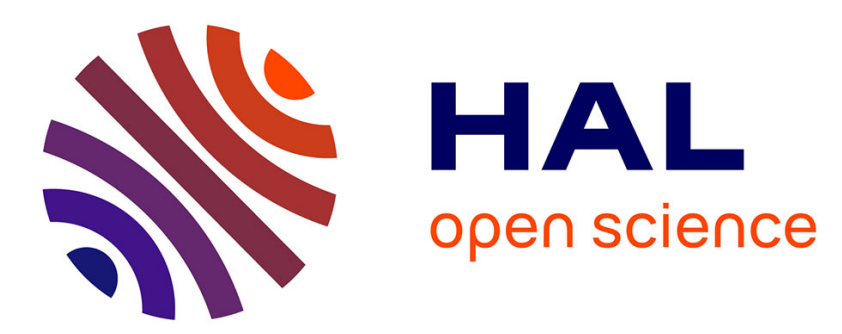

\title{
Trapped modes in thin and infinite ladder like domains. Part 2: asymptotic analysis and numerical application
} Bérangère Delourme, Sonia Fliss, Patrick Joly, Elizaveta Vasilevskaya

\section{To cite this version:}

Bérangère Delourme, Sonia Fliss, Patrick Joly, Elizaveta Vasilevskaya. Trapped modes in thin and infinite ladder like domains. Part 2: asymptotic analysis and numerical application. Asymptotic Analysis, In press. hal-01822437v2

\section{HAL Id: hal-01822437 \\ https://hal.science/hal-01822437v2}

Submitted on 5 Dec 2019

HAL is a multi-disciplinary open access archive for the deposit and dissemination of scientific research documents, whether they are published or not. The documents may come from teaching and research institutions in France or abroad, or from public or private research centers.
L'archive ouverte pluridisciplinaire HAL, est destinée au dépôt et à la diffusion de documents scientifiques de niveau recherche, publiés ou non, émanant des établissements d'enseignement et de recherche français ou étrangers, des laboratoires publics ou privés. 


\title{
Trapped modes in thin and infinite ladder like domains. Part 2 : asymptotic analysis and numerical application
}

\author{
Bérangère Delourme ${ }^{\mathrm{a}, *}$, Sonia Fliss ${ }^{\mathrm{b}}$, Patrick Joly ${ }^{\mathrm{b}}$ and Elizaveta Vasilevskaya ${ }^{\mathrm{a}}$ \\ ${ }^{a}$ LAGA, Universite Paris 13, Villetaneuse, France \\ E-mail:delourme@math.univ-paris13.fr \\ b POEMS, CNRS,INRIA,ENSTA Paris, Institut Polytechnique de Paris, Palaiseau, France \\ E-mails: sonia.fliss@ensta-paris.fr,patrick.joly@inria.fr
}

\begin{abstract}
We are interested in a 2D propagation medium obtained from a localized perturbation of a reference homogeneous periodic medium. This reference medium is a "thick graph", namely a thin structure (the thinness being characterized by a small parameter $\varepsilon>0$ ) whose limit (when $\varepsilon$ tends to 0 ) is a periodic graph. The perturbation consists in changing only the geometry of the reference medium by modifying the thickness of one of the lines of the reference medium. In the first part of this work, we proved that such a geometrical perturbation is able to produce localized eigenmodes (the propagation model under consideration is the scalar Helmholtz equation with Neumann boundary conditions). This amounts to solving an eigenvalue problem for the Laplace operator in an unbounded domain. We used a standard approach of analysis that consists in (1) find a formal limit of the eigenvalue problem when the small parameter tends to 0 , here the formal limit is an eigenvalue problem for a second order differential operator along a graph; (2) proceed to an explicit calculation of the spectrum of the limit operator; (3) deduce the existence of eigenvalues as soon as the thickness of the ladder is small enough. The objective of the present work is to complement the previous one by constructing and justifying a high order asymptotic expansion of these eigenvalues (with respect to the small parameter $\varepsilon$ ) using the method of matched asymptotic expansions. In particular, the obtained expansion can be used to compute a numerical approximation of the eigenvalues and of their associated eigenvectors. An algorithm to compute each term of the asymptotic expansion is proposed. Numerical experiments validate the theoretical results.
\end{abstract}

Keywords: spectral theory, periodic media, quantum graphs, matched asymptotic expansion

\section{Summary of Part 1 and Main results of Part 2}

This article is the sequel of [1] and we refer the reader to its introduction for the motivation of the study and related bibliographical comments. We choose to go directly to the heart of the subject and to give below a brief recap about the problem under consideration, then to give a summary of the main results of [1] in Sections 1.1 and 1.2. Finally, we state the main result of the present paper in Section 1.3.

Let $\Omega_{\varepsilon}$ be a homogeneous periodic domain consisting of the infinite band $\{(x, y) \in \mathbb{R} \times(-L / 2, L / 2)\}$ of height $L>0$ minus an infinite set of equispaced similar rectangular obstacles (see Figure 1). The domain $\Omega_{\varepsilon}$ is 1-periodic with respect to the variable $x$. The distance between two consecutive obstacles is equal to the distance from the obstacles to the boundary of the band and is denoted by $\varepsilon$.

\footnotetext{
${ }^{*}$ Corresponding author. E-mail: sonia.fliss@ensta-paris.fr.
} 

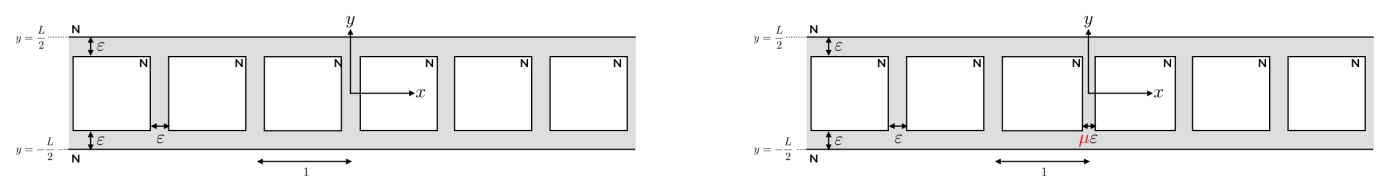

Fig. 1. The unperturbed periodic ladder (left) . The Perturbed ladder (right)

Starting from the periodic domain $\Omega_{\varepsilon}$, we introduce a local perturbation by changing the distance between two consecutive obstacles from $\varepsilon$ to $\mu \varepsilon, \mu>0$ (i.e by modifying the size of two consecutive obstacles of $(1-\mu) \varepsilon / 2)$ see Figure 1 (right) in the case where $\mu \in(0,1))$. It corresponds to modify the width of the vertical rung of the ladder from $|x|<\varepsilon / 2$ to $|x|<\mu \varepsilon / 2$. The corresponding domain is denoted $\Omega_{\varepsilon}^{\mu}$. We wonder whether such a perturbation creates so called localized modes, that is to say harmonic in time functions of the form

$$
u(x, y, t)=v(x, y) e^{i \omega t}, \quad v \in L_{2}\left(\Omega_{\varepsilon}\right), \quad \omega \in \mathbb{R},
$$

that satisfy the wave equation with homogeneous Neumann boundary condition

$$
\frac{\partial^{2} u}{\partial t^{2}}-\Delta u=0, \quad \partial_{n} u=0 \quad \text { on } \quad \partial \Omega_{\varepsilon} .
$$

\subsection{Mathematical formulation of the problem}

\subsubsection{The operator $A_{\varepsilon}^{\mu}$}

Injecting (1) into (2), one can easily see that the construction of localized modes turns out to solve the following eigenvalue problem for the function $v$ :

$$
-\Delta v=\omega^{2} v \text { in } \Omega_{\varepsilon}, \quad \partial_{n} v=0 \text { in } \partial \Omega_{\varepsilon} .
$$

It consequently leads us to investigate the spectrum (and more precisely the eigenvalues) of the selfadjoint and positive operator $A_{\varepsilon}^{\mu}$, acting in the space $L_{2}\left(\Omega_{\varepsilon}^{\mu}\right)$ :

$$
A_{\varepsilon}^{\mu} u=-\Delta u, \quad D\left(A_{\varepsilon}^{\mu}\right)=\left\{u \in H^{1}\left(\Omega_{\varepsilon}^{\mu}\right), \Delta u \in L_{2}\left(\Omega_{\varepsilon}^{\mu}\right),\left.\partial_{n} u\right|_{\partial \Omega_{\varepsilon}^{\mu}}=0\right\} .
$$

Based on an asymptotic approach, the spectrum of the operators $A_{\varepsilon}^{\mu}$ is investigated in [1]. In particular, it is shown that for $\mu \in(0,1)$, and for $\varepsilon>0$ sufficiently small, the operator $A_{\varepsilon}^{\mu}$ has eigenvalues. The objective of the present paper is to complement the aforementioned work by constructing a high order asymptotics of these eigenvalues.

\subsubsection{The decomposition of the operator $A_{\varepsilon}^{\mu}$ into its symmetric and antisymmetric components}

To study the operator $A_{\varepsilon}^{\mu}$, it is convenient to decompose it as the sum of its symmetric and antisymmetric parts. Denoting $L_{2, s}\left(\Omega_{\varepsilon}^{\mu}\right)$ and $L_{2, a}\left(\Omega_{\varepsilon}^{\mu}\right)$ the subspaces of $L_{2}\left(\Omega_{\varepsilon}^{\mu}\right)$ consisting of functions respectively symmetric and antisymmetric (with respect to the axis $y=0$ ), we have

$$
L_{2}\left(\Omega_{\varepsilon}^{\mu}\right)=L_{2, s}\left(\Omega_{\varepsilon}^{\mu}\right) \oplus L_{2, a}\left(\Omega_{\varepsilon}^{\mu}\right) .
$$

The operator $A_{\varepsilon}^{\mu}$ is then decomposed as follows, where $A_{\varepsilon, s}^{\mu}$ and $A_{\varepsilon, a}^{\mu}$ are both self-adjoint and positive

$$
A_{\varepsilon}^{\mu}=A_{\varepsilon, s}^{\mu} \oplus A_{\varepsilon, a}^{\mu}, \quad \text { with } \quad A_{\varepsilon, s}^{\mu}=\left.A_{\varepsilon}^{\mu}\right|_{L_{2, s}\left(\Omega_{\varepsilon}^{\mu}\right)}, \quad \text { and } \quad A_{\varepsilon, a}^{\mu}=\left.A_{\varepsilon}^{\mu}\right|_{L_{2, a}\left(\Omega_{\varepsilon}^{\mu}\right)},
$$

In this paper, we shall restrict ourselves to the study of the spectrum of the symmetric operator $A_{\varepsilon, s}^{\mu}$. Naturally, we could study the antisymmetric one as well. 


\subsection{The limit problem: spectral problem on the graph}

As might be expected, the investigation of the spectrum of $A_{\varepsilon, s}^{\mu}$ relies on the investigation of the spectrum of a limit operator denoted by $\mathcal{A}_{s}^{\mu}$ defined on a graph $\mathcal{G}$ obtained as the geometrical limit of the domain $\Omega_{\varepsilon}^{\mu}$ as $\varepsilon$ tends to 0 . In this section, we define the limit operators $\mathcal{A}_{s}^{\mu}$, and we remind important characteristics of its spectrum, established in [1].

\subsubsection{The limit operators $\mathcal{A}^{\mu}, \mathcal{A}_{s}^{\mu}$ and $\mathcal{A}_{a}^{\mu}$}

The limit periodic graph $\mathcal{G}$.

Let us first introduce some notation associated with the limit periodic graph $\mathcal{G}=\bigcap_{\varepsilon>0} \Omega_{\varepsilon}^{\mu}$ represented on Figure 2. We denote by $e_{j}$ the vertical edge $e_{j}=\{j\} \times(-L / 2, L / 2)$. The edge $e_{0}$ corresponds to the limit of the perturbed rung $\{|x|<\mu \varepsilon / 2\}$. For all $j$, the upper end of the edge $e_{j}$ is denoted by $M_{j}^{+}$and the lower one by $M_{j}^{-}$. The horizontal edge joining $M_{j}^{ \pm}$and $M_{j+1}^{ \pm}$is denoted by $e_{j+\frac{1}{2}}^{ \pm}=(j, j+1) \times\{ \pm L / 2\}$.

The sets of all vertices and all edges of the graph are then

$$
\mathcal{M}=\left\{M_{j}^{ \pm}\right\}_{j \in \mathbb{Z}}, \quad \mathcal{E}=\left\{e_{j}, e_{j+\frac{1}{2}}^{ \pm}\right\}_{j \in \mathbb{Z}}
$$

and we denote by $\mathcal{E}(M)$ the set of all the edges of the graph containing the vertex $M$.

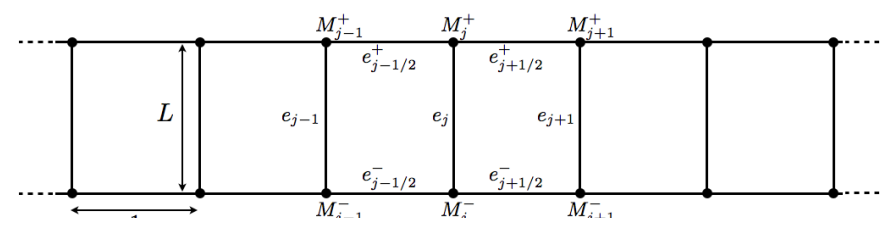

Fig. 2. Limit graph $\mathcal{G}$

Weighted functional spaces on $\mathcal{G}$. If $u$ is a function defined on $\mathcal{G}$ we will use the following notation

$$
\mathbf{u}_{j}^{ \pm}=u\left(M_{j}^{ \pm}\right), \quad u_{j}(y)=\left.u\right|_{e_{j}}, \quad u_{j+\frac{1}{2}}^{ \pm}(x)=\left.u\right|_{e_{j+\frac{1}{2}}^{ \pm}} .
$$

Let $w^{\mu}: \mathcal{E} \longrightarrow \mathbb{R}^{+}$dsuch that $w^{\mu}\left(e_{0}\right)=\mu, \quad w^{\mu}(e)=1, \quad \forall e \in \mathcal{E}, e \neq e_{0}$.

Let us now introduce the following weighted functional spaces

$$
\begin{aligned}
& L_{2}^{\mu}(\mathcal{G})=\left\{u / u \in L_{2}(e), \forall e \in \mathcal{E} ; \quad\|u\|_{L_{2}^{\mu}(\mathcal{G})}^{2}=\sum_{e \in \mathcal{E}} w^{\mu}(e)\|u\|_{L_{2}(e)}^{2}<\infty\right\}, \\
& H^{1}(\mathcal{G})=\left\{u \in L_{2}^{\mu}(\mathcal{G}) / u \in C(\mathcal{G}) ; \quad u \in H^{1}(e), \forall e \in \mathcal{E} ; \quad\|u\|_{H^{1}(\mathcal{G})}^{2}=\sum_{e \in \mathcal{E}}\|u\|_{H^{1}(e)}^{2}<\infty\right\}, \\
& H^{2}(\mathcal{G})=\left\{u \in L_{2}^{\mu}(\mathcal{G}) / u \in C(\mathcal{G}) ; \quad u \in H^{2}(e), \forall e \in \mathcal{E} ; \quad\|u\|_{H^{2}(\mathcal{G})}^{2}=\sum_{e \in \mathcal{E}}\|u\|_{H^{2}(e)}^{2}<\infty\right\},
\end{aligned}
$$

where $C(\mathcal{G})$ denotes the space of continuous functions on $\mathcal{G}$. 
Definition of the limit operators $\mathcal{A}^{\mu}, \mathcal{A}_{s}^{\mu}$ and $\mathcal{A}_{a}^{\mu}$. As known since the work of [2-4], the limit operator $\mathcal{A}^{\mu}$ acting on $L_{2}^{\mu}(\mathcal{G})$ is defined as follows: denoting by $u_{e}$ the restriction of $u$ to $e$,

$$
\left(\mathcal{A}^{\mu} u\right)_{e}=-u_{e}^{\prime \prime}, \forall e \in \mathcal{E}, \quad D\left(\mathcal{A}^{\mu}\right)=\left\{u \in H^{2}(\mathcal{G}) / \sum_{e \in \mathcal{E}(M)} w^{\mu}(e) u_{e}^{\prime}(M)=0, \forall M \in \mathcal{M}\right\},
$$

where $u_{e}^{\prime}(M)$ stands for the derivative of the function $u_{e}$ at the point $M$ in the outgoing direction. The vertex relations in (8) are called Kirchhoff's conditions. Note that they all have an identical expression except at the vertices $M_{0}^{ \pm}$. We remark that the perturbation, which results from a geometrical modification of the domain for the initial operator, is taken into account at the limit by means of the Kirchhoff's conditions at the vertices $M_{0}^{ \pm}$. The operator $\mathcal{A}^{\mu}$ is indeed positive and self-adjoint (cf. [5, Section 3.3]).

As for the ladder, denoting $L_{2, s}^{\mu}(\mathcal{G})$ and $L_{2, a}^{\mu}(\mathcal{G})$ the subspaces of $L_{2}^{\mu}(\mathcal{G})$ consisting of functions respectively symmetric and antisymmetric (with respect to the axis $y=0$ ) we have

$$
L_{2}^{\mu}(\mathcal{G})=L_{2, s}^{\mu}(\mathcal{G}) \oplus L_{2, a}^{\mu}(\mathcal{G}) .
$$

Thus, the operator $\mathcal{A}^{\mu}$ can be decomposed into the orthogonal sum where $\mathcal{A}_{s}^{\mu}$ and $\mathcal{A}_{a}^{\mu}$ are again selfadjoint positive operators

$$
\mathcal{A}^{\mu}=\mathcal{A}_{s}^{\mu} \oplus \mathcal{A}_{a}^{\mu}, \quad \text { with } \quad \mathcal{A}_{s}^{\mu}=\left.\mathcal{A}^{\mu}\right|_{L_{2, s}^{\mu}(\mathcal{G})}, \quad \text { and } \quad \mathcal{A}_{a}^{\mu}=\left.\mathcal{A}^{\mu}\right|_{L_{2, a}^{\mu}(\mathcal{G})},
$$

\subsubsection{The spectrum of $\mathcal{A}_{s}^{\mu}$}

The operator $\mathcal{A}_{s}^{\mu}$ being self-adjoint, its spectrum consists of its essential spectrum $\sigma_{\text {ess }}\left(\mathcal{A}_{s}^{\mu}\right)$ and its discrete spectrum $\sigma_{d}\left(\mathcal{A}_{s}^{\mu}\right)$. It is well-known that $\sigma_{\text {ess }}\left(\mathcal{A}_{s}^{\mu}\right)$ coincides with the spectrum of the periodic operator $\mathcal{A}_{s}:=\mathcal{A}_{s}^{\mu}$ for $\mu=1$ (see e.g. [6, Theorem 4, Chapter 9]) and has a band-gap structure [7-9]:

$$
\sigma_{\text {ess }}\left(\mathcal{A}_{s}^{\mu}\right)=\sigma\left(\mathcal{A}_{s}\right)=\bigcup_{n \in \mathbb{N}}\left[a_{n}, b_{n}\right]
$$

where $a_{0} \geqslant 0, a_{n}<a_{n+1}$ and $a_{n} \leqslant b_{n}$. In general, the segments $\left[a_{n}, b_{n}\right]$ may overlap. If, for some $n \in \mathbb{N}$, $b_{n}<a_{n+1}$, the open interval $] b_{n}, a_{n+1}$ [ is called a gap of the essential spectrum operator $\mathcal{A}_{s}^{\mu}$.

The following proposition, based on explicit characterizations of $\sigma_{\text {ess }}\left(\mathcal{A}_{s}^{\mu}\right)$ and $\sigma_{\mathrm{d}}\left(\mathcal{A}_{s}^{\mu}\right)$, is proved in [1, Proposition 5 and Theorem 1]:

\section{Proposition 1.}

(1) The essential spectrum of the operator $\mathcal{A}_{s}^{\mu}$ has infinitely many gaps whose ends tend to infinity.

(2) For $\mu \geqslant 1$, the discrete spectrum of the operator $\mathcal{A}_{s}^{\mu}$ is empty, while for $\mu \in(0,1)$, the operator $\mathcal{A}_{s}^{\mu}$ has exactly one or two eigenvalue( $s)$ in each of its gaps, each eigenvalue being simple.

To show the last item, we use the characterization of the spectrum

$$
\lambda \in \sigma_{\mathrm{d}}\left(\mathcal{A}_{s}^{\mu}\right) \Leftrightarrow|g(\sqrt{\lambda})|>1 \text { and } \mu=1-\sqrt{\frac{g^{2}(\sqrt{\lambda})-1}{(g(\sqrt{\lambda})+\cos \sqrt{\lambda})^{2}}}
$$


where $\forall \omega>0, \quad g(\omega)=-\cos (\omega)+\frac{\sin (\omega) \tan (\omega L / 2)}{2}$.

An associated eigenvector $u$ is given, on the horizontal edges $e_{j+1 / 2}^{ \pm}(j \in \mathbb{Z})$, by

$$
u_{j+\frac{1}{2}}(s)=r(\sqrt{\lambda})^{|j|}(\sin (\sqrt{\lambda}(1-s))+r(\sqrt{\lambda}) \sin (\sqrt{\lambda} s)) / \sin \sqrt{\lambda}, \quad s:=x-j \in[0,1],
$$

while, on the vertical edges $e_{j}(j \in \mathbb{Z})$, it is given by

$$
u_{j}(y)=r(\sqrt{\lambda})^{|j|} \cos (\sqrt{\lambda} y) / \cos (\sqrt{\lambda} L / 2), \quad y \in[-L / 2, L / 2],
$$

where $r(\sqrt{\lambda})$ is the unique root in $(-1,1)$ of the following characteristic equation

$$
r^{2}+2 g(\sqrt{\lambda}) r+1=0 .
$$

\subsection{Main result}

Before stating the main result of this paper, let us remind first the result, already proven for instance in [4], which states the convergence of the essential spectrum of the operator $A_{\varepsilon, s}^{\mu}$ to the essential spectrum of $\mathcal{A}_{s}^{\mu}$. More precisely, let $(a, b)$ be a gap of the operator $\mathcal{A}_{s}^{\mu}$ on the limit graph $\mathcal{G}$ then, there exists $\varepsilon_{0}>0$ such that if $\varepsilon<\varepsilon_{0}$ the operator $A_{\varepsilon, s}^{\mu}$ has a gap $\left(a^{\varepsilon}, b^{\varepsilon}\right)$ whose extremities satisfy

$$
a^{\varepsilon}=a+O(\varepsilon) \quad \text { and } \quad b^{\varepsilon}=b+O(\varepsilon) .
$$

This means that for some $C_{1}>0$ and for any $\varepsilon<\varepsilon_{0}$

$$
\sigma_{e s s}\left(A_{\varepsilon, s}^{\mu}\right) \cap\left[a+C_{1} \varepsilon, b-C_{1} \varepsilon\right]=\emptyset .
$$

In [1, Theorem 1], we have shown that for $\mu \in(0,1)$, and for $\varepsilon>0$ sufficiently small the discrete spectrum of $A_{\varepsilon, s}^{\mu}$ is not empty. More precisely, for $\mu \in(0,1)$, the discrete spectrum of $\mathcal{A}_{s}^{\mu}$ is not empty and if $\lambda^{(0)} \in(a, b)$ is an eigenvalue of this operator, then there exists $0<\varepsilon_{1}<\varepsilon_{0}$ such that if $\varepsilon<\varepsilon_{1}$ the operator $A_{\varepsilon, s}^{\mu}$ has an eigenvalue $\lambda^{\varepsilon}$ inside the gap $\left(a^{\varepsilon}, b^{\varepsilon}\right)$. Moreover,

$$
\lambda^{\varepsilon}=\lambda^{(0)}+O(\sqrt{\varepsilon}) .
$$

The present paper complements the result of [1] by obtaining and constructing an asymptotic expansion of these eigenvalues at any order with respect to the parameter $\varepsilon$.

Theorem 1. Let $\lambda^{(0)} \in(a, b)$ be an eigenvalue of the operator $\mathcal{A}_{s}^{\mu}$ and for $\varepsilon$ small enough, $\lambda^{\varepsilon}$ the unique eigenvalue of the operator $A_{\varepsilon, s}^{\mu}$ satisfying (16). Then, there exists a real sequence $\left(\lambda^{(k)}\right)_{k \in \mathbb{N}^{*}}$, which is constructed inductively with the help of an algorithm that is presented in detail in Section 5, such that

$$
\forall n \in \mathbb{N}, \quad \lambda^{\varepsilon}=\sum_{k=0}^{n} \varepsilon^{k} \lambda^{(k)}+O\left(\varepsilon^{n+1}\right) .
$$


Remark 1. Note that as every eigenvalue of the operators $\mathcal{A}_{s}^{\mu}$ is simple (as established in Prop. 1), for $\varepsilon$ small enough, $\lambda^{\varepsilon}$ is a simple eigenvalue of $A_{\varepsilon, s}^{\mu}$, see [10]. As soon as one obtains such asymptotic expansions for these simple eigenvalues, it is also possible to deduce an asymptotic expansion for a prescribed associated eigenvector (see for instance [11, Part 4]).

We point out that the main novelty of the previous result lies in the determination of a high order asymptotic expansion of the eigenvalues of $A_{\varepsilon, s}^{\mu}$, and we use it for numerical computations. The convergence of the spectrum of $A_{\varepsilon, s}^{\mu}$ toward the spectrum of $\mathcal{A}_{s}^{\mu}$ has been proved in [10]. The case of 'fattened' compact graphs with more general boundary conditions has been investigated in [12] while the specific case of Dirichlet boundary conditions was also studied in [13]. A more complete presentation of related bibliographical references can be found in [1].

\section{Methodology of the proof and asymptotic expansion ansatz}

\subsection{Methodology of the proof}

The proof of Theorem 1 relies on the following lemma.

Lemma 1. Let $\lambda^{(0)} \in(a, b)$ be an eigenvalue of $\mathcal{A}_{s}^{\mu}$. Suppose that there exist two sequences of real numbers $\left(\lambda^{(m)}\right)_{m \in \mathbb{N}^{*}}$ and $\left(\alpha_{m}\right)_{m \in \mathbb{N}^{*}}$, with

$$
0<\alpha_{m} \leqslant m+1 \text { and } \lim _{m \rightarrow+\infty} \alpha_{m}=+\infty
$$

and a sequence $u^{\varepsilon, m} \in H_{s}^{1}\left(\Omega_{\varepsilon}^{\mu}\right)$ such that, for any $v \in H_{s}^{1}\left(\Omega_{\varepsilon}^{\mu}\right)$

$$
\left|\int_{\Omega_{\varepsilon}^{\mu}}\left(\nabla u^{\varepsilon, m} \nabla v-\lambda^{\varepsilon, m} u^{\varepsilon, m} v\right)\right| \leqslant C \varepsilon^{\alpha_{m}}\left\|u^{\varepsilon, m}\right\|_{H^{1}\left(\Omega_{\varepsilon}^{\mu}\right)}\|v\|_{H^{1}\left(\Omega_{\varepsilon}^{\mu}\right)}, \quad \text { where } \lambda^{\varepsilon, m}=\sum_{k=0}^{m} \varepsilon^{k} \lambda^{(k)},
$$

Then there exists at least one eigenvalue $\lambda^{\varepsilon}$ of $A_{\varepsilon, s}^{\mu}$ such that

$$
\forall n \in \mathbb{N}, \quad \lambda^{\varepsilon}=\sum_{k=0}^{n} \varepsilon^{k} \lambda^{(k)}+O\left(\varepsilon^{n+1}\right) .
$$

Proof. Assume that the sequences $\left(u^{\varepsilon, m}\right)_{m \in \mathbb{N}}$ and $\left(\lambda^{\varepsilon, m}\right)_{m \in \mathbb{N}}$ satisfying (19) are constructed and let $n \in \mathbb{N}$. First, let us choose $m_{n} \in \mathbb{N}$ such that $\alpha_{m_{n}} \geqslant n+1$ (this is possible because of (18)). By adapting the Lemma 4 for [14] (see the Appendix A in [15] for a proof in a general case), (19) provides an estimate of the distance from $\lambda^{\varepsilon, m_{n}}$ to the spectrum of $A_{\varepsilon, s}^{\mu}$ :

$$
\operatorname{dist}\left(\sigma\left(A_{\varepsilon, s}^{\mu}\right), \lambda^{\varepsilon, m_{n}}\right) \leqslant \widetilde{C} \varepsilon^{\alpha_{m_{n}}} \leqslant \widetilde{C} \varepsilon^{n+1},
$$

with some constant $\widetilde{C}$ which is related to $C$ but does not depend on $\varepsilon$. Of course,

$$
(20) \Longleftrightarrow \sigma\left(A_{\varepsilon, s}^{\mu}\right) \cap I_{n}^{\varepsilon} \neq \emptyset, \quad I_{n}^{\varepsilon}:=\left[\lambda^{\varepsilon, m_{n}}-\widetilde{C} \varepsilon^{n+1}, \lambda^{\varepsilon, m_{n}}+\widetilde{C} \varepsilon^{n+1}\right] .
$$


Since $\lambda^{(0)} \in(a, b)$, for $\varepsilon$ small enough, the interval $I_{n}^{\varepsilon}$ (which tends to $\lambda^{(0)}$ when $\varepsilon$ goes to 0 ) is included in $\left[a+C_{1} \varepsilon, b-C_{1} \varepsilon\right]$ (which tends to $[a, b]$ ) and thus does not intersect $\sigma_{e s s}\left(A_{\varepsilon, s}^{\mu}\right)$ according to (15) again. As a consequence of (20), $\sigma_{d}\left(A_{\varepsilon, s}^{\mu}\right)$ denoting the discrete spectrum of $A_{\varepsilon, s}^{\mu}$

$$
\sigma_{d}\left(A_{\varepsilon, s}^{\mu}\right) \cap\left[\lambda^{\varepsilon, m_{n}}-\widetilde{C} \varepsilon^{n+1}, \lambda^{\varepsilon, m_{n}}+\widetilde{C} \varepsilon^{n+1}\right] \neq \emptyset,
$$

Thus, for small $\varepsilon$, there is at least one eigenvalue $\lambda_{\varepsilon}$ of $A_{\varepsilon, s}^{\mu}$ at a distance to $\lambda^{\varepsilon, m_{n}}$ of order $\varepsilon^{n+1}$, i.e.

$$
\left|\lambda_{\varepsilon}-\lambda^{\varepsilon, m_{n}}\right| \leqslant C \varepsilon^{n+1}
$$

The end of the proof of (17) then follows directly from the triangular inequality since

$$
\left|\lambda_{\varepsilon}-\lambda^{\varepsilon, n}\right| \leqslant\left|\lambda_{\varepsilon}-\lambda^{\varepsilon, m_{n}}\right|+\left|\lambda^{\varepsilon, m_{n}}-\lambda^{\varepsilon, n}\right| \leqslant C\left|\lambda_{\varepsilon}-\lambda^{\varepsilon, m_{n}}\right|+\sum_{k=n+1}^{m_{n}} \varepsilon^{k}\left|\lambda^{(k)}\right| \leqslant C \varepsilon^{n+1} .
$$

The function $u^{\varepsilon, m}$ is called a pseudo-mode. This pseudo-mode and the associated expansion $\lambda^{\varepsilon, m}$ are constructed thanks to a formal asymptotic expansion of an eigenpair $\left(u^{\varepsilon}, \lambda^{\varepsilon}\right)$ of the following problem

$$
\begin{aligned}
& \text { Find } u^{\varepsilon} \in H^{1}\left(\Omega_{\varepsilon}^{\mu}\right) \text { s. t. } u^{\varepsilon}(x, y)=u^{\varepsilon}(x,-y), \forall(x, y) \in \Omega_{\varepsilon}^{\mu}, u^{\varepsilon} \neq 0, \text { and } \lambda^{\varepsilon} \in \mathbb{R}^{+}, \\
& \Delta u^{\varepsilon}+\lambda^{\varepsilon} u^{\varepsilon}=0 \quad \text { in } \quad \Omega_{\varepsilon}^{\mu}, \quad \partial_{n} u^{\varepsilon}=0 \quad \text { on } \partial \Omega_{\varepsilon}^{\mu},
\end{aligned}
$$

This asymptotic expansion will be constructed by induction starting from an eigenvalue $\lambda^{(0)} \in(a, b)$ of the operator $\mathcal{A}_{s}^{\mu}$ and an associated eigenvector $u^{(0)}$.

Due to the multiscale nature of the problem, it is not possible to construct a simple asymptotic expansion of $u^{\varepsilon}$ that would be valid in the whole domain $\Omega_{\varepsilon}^{\mu}$. We need to distinguish two asymptotic expansions of $u^{\varepsilon}$. The first one, describing the overall behaviour of $u^{\varepsilon}$ far from the junctions, is expressed by means of the longitudinal coordinate $s(s=x-j$ for the j-th horizontal thin slit and $s=y$ for the vertical thin slits) and is called the far field expansion. The second one is the near field expansion and is used to approximate $u^{\varepsilon}$ in the neighborhood of each junction. Thus, it is expressed by means of the fast variables $((x-j) / \varepsilon,(y+L / 2) / \varepsilon)$ near the j-th junction and is defined on a normalized unperturbed junction for $j \neq 0$ and a normalized perturbed junction for $j=0$. Since both expansions are meant to be two approximations of the same function $u^{\varepsilon}$, they have to satisfy some matching conditions in some intermediate zones. This method is often called Matched Asymptotic Expansion. For complete and detailed descriptions of the method, we refer the reader to [16], [17] and [11] (cf. Part IV dedicated to eigenvalue problems). See also [18] for a recent application of the method to a spectral problem. See also [19, 20] and [21, Chapter 8] for another asymptotic study of similar periodic skeletal structures.

In Section 2.2, we give the ansatz for the far field and the near field expansions as sums of far field and near field terms indexed by $n \in \mathbb{N}$ and derive the problems (defined inductively on $n$ ) satisfied by these far field and near field terms. We give the matching conditions in Section 2.3. We study in Sections 3.1-3.2 and in Section 3.3 the well posedness of the problems satisfied by the near field terms and the far field terms respectively. We explain the algorithm of construction of each term in Section 4 and finally 
prove Theorem 1 by establishing (19) in Section 5.

Before entering the details, let us introduce some notations. The function $u^{\varepsilon}$ being even in $y$, it suffices to construct an asymptotic expansion of $u^{\varepsilon}$ on the lower half part $\Omega_{\varepsilon}^{\mu,-}$ of $\Omega_{\varepsilon}^{\mu}$ (comb shape domain):

$$
\Omega_{\varepsilon}^{\mu,-}=\left\{(x, y) \in \Omega_{\varepsilon}^{\mu} \quad \text { s.t. } y<0\right\} .
$$

As represented on Figure 3, we denote by $\mathcal{E}_{j+\frac{1}{2}}^{\varepsilon,-}, j \in \mathbb{Z}$, the horizontal slits of the domain $\Omega_{\varepsilon}^{\mu,-}$

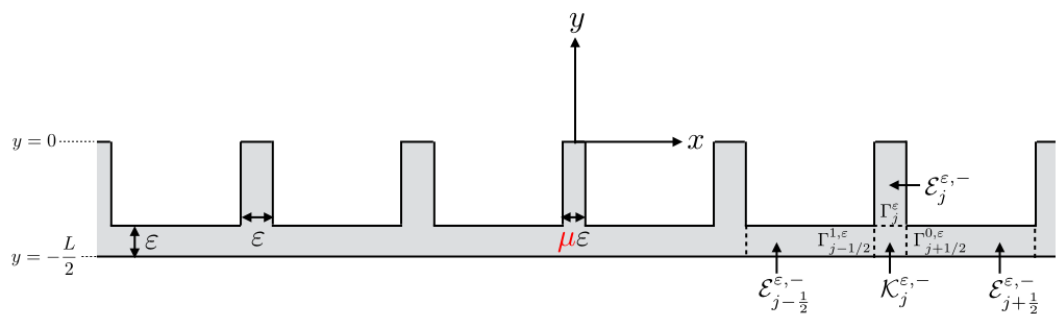

Fig. 3. The domain $\Omega_{\varepsilon}^{\mu,-}$

$$
\mathcal{E}_{j+\frac{1}{2}}^{\varepsilon,-}=\left(j+\varepsilon \mu_{j} / 2,(j+1)-\varepsilon \mu_{j+1} / 2\right) \times(-L / 2,-L / 2+\varepsilon),
$$

by $\mathcal{E}_{j}^{\varepsilon,-}$ its vertical slits $\mathcal{E}_{j}^{\varepsilon,-}=\left(j-\varepsilon \mu_{j} / 2, j+\varepsilon \mu_{j} / 2\right) \times(-L / 2+\varepsilon, 0)$.

The domains $\mathcal{K}_{j}^{\varepsilon,-}$ are the junctions $\mathcal{K}_{j}^{\varepsilon,-}=\left(j-\varepsilon \mu_{j} / 2, j+\varepsilon \mu_{j} / 2\right) \times(-L / 2,-L / 2+\varepsilon)$ where for all $j \in \mathbb{Z}, \mu_{j}=1$ if $j \neq 0$ and $\mu_{0}=\mu$.

\subsection{Asymptotic expansions : ansatz and equations}

From now on, , $\lambda^{(0)} \in(a, b)$ is an eigenvalue of $\mathcal{A}_{s}^{\mu}$ and $u^{(0)}$ an associated eigenvector :

$$
u^{(0)} \in D\left(\mathcal{A}_{s}^{\mu}\right), \quad \mathcal{A}_{s}^{\mu} u^{(0)}=\lambda^{(0)} u^{(0)}
$$

i.e. denoting $\left.\forall j \in \mathbb{Z} u_{j+\frac{1}{2}}^{(0)} \equiv u^{(0)}\right|_{e_{j+1 / 2}^{ \pm}}$and $\left.u_{j}^{(0)} \equiv u^{(0)}\right|_{e_{j}}$ (using the notations of Section 1.2.1)

$$
\forall j \in \mathbb{Z}, \quad \begin{cases}\partial_{s}^{2} u_{j+\frac{1}{2}}^{(0)}(s)+\lambda^{(0)} u_{j+\frac{1}{2}}^{(k)}(s)=0, & s=x-j \in[0,1], \\ \partial_{y}^{2} u_{j}^{(0)}(y)+\lambda^{(0)} u_{j}^{(0)}(y)=0, & y \in[-L / 2,0], \\ \partial_{y} u_{j}^{(0)}(0)=0, & \\ u_{j-\frac{1}{2}}^{(0)}(1)=u_{j}^{(0)}(-L / 2)=u_{j+\frac{1}{2}}^{(0)}(0), & \\ \partial_{s} u_{j+\frac{1}{2}}^{(0)}(0)-\partial_{s} u_{j-\frac{1}{2}}^{(0)}(1)+\mu_{j} \partial_{y} u_{j}^{(0)}(-L / 2)=0 . & \end{cases}
$$


where we denote $\partial_{s}$ (resp. $\partial_{y}$ ) the derivative with respect to $s$ (resp. to $y$ ).

To start the construction of the asymptotic expansion, we have to fix $u^{(0)}$. We have chosen $u^{(0)}$ such that $u_{0}^{(0)}(-L / 2)=1$, namely, $\forall j \in \mathbb{Z}$,

$$
\begin{array}{ll}
u_{j+\frac{1}{2}}^{(0)}(s)=r^{|j|} \frac{\sin \left(\sqrt{\lambda^{(0)}}(1-s)\right)}{\sin \sqrt{\lambda^{(0)}}}+r^{|j+1|} \frac{\sin \left(\sqrt{\lambda^{(0)}} s\right)}{\sin \sqrt{\lambda^{(0)}}}, & s=x-j \in[0,1], \\
u_{j}^{(0)}(y)=r^{|j|} \frac{\cos \left(\sqrt{\lambda^{(0)}} y\right)}{\cos \left(\sqrt{\lambda^{(0)}} L / 2\right)}, & y \in[-L / 2, L / 2] .
\end{array}
$$

Here $r$ stands for $r\left(\sqrt{\lambda^{(0)}}\right)$ defined in (13). Note that $u^{(0)}$ is exponentially decaying with $|x|$.

Remark 2. Choosing another eigenvector $u^{(0)}$ leads obviously to the asymptotic expansion of a different $u_{\varepsilon}$ but this does not change the asymptotic expansion of $\lambda^{\varepsilon}$ (see Remark 7).

We propose an asymptotic expansion for $\lambda^{\varepsilon}$ and $u^{\varepsilon}$ solution of (21) constructed by induction starting from $\lambda^{(0)}$ and $u^{(0)}$. In the following, $O\left(\varepsilon^{\infty}\right)$ will always denote a remainder that (formally) decays more rapidly that any power of $\varepsilon$. We first suppose a formal power series expansion for the eigenvalue:

$$
\lambda^{\varepsilon}=\sum_{k \in \mathbb{N}} \varepsilon^{k} \lambda^{(k)}+O\left(\varepsilon^{\infty}\right)
$$

Remark 3. Throughout the rest of the paper, we shall extend the above convention: any quantity indexed by $k$ with $k<0$ is automatically 0 .

Mimicking the approach of [22-24], we use the following ansatz (see Figure 4)

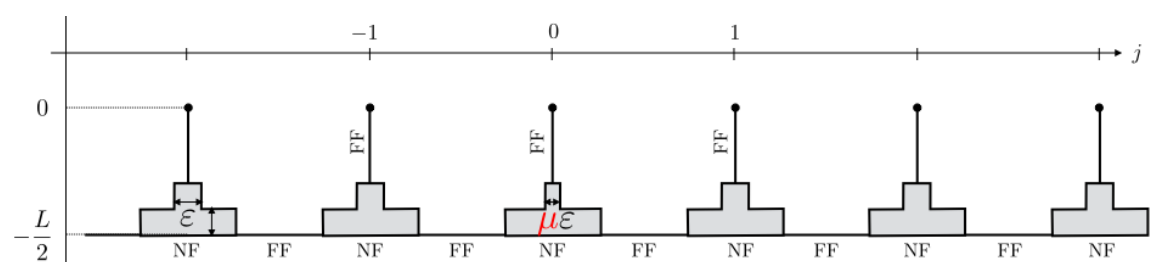

Fig. 4. Schematic representation of the asymptotic expansion (NF: near field, FF: far field)

- Far field asymptotic expansion: in the horizontal thin slits $\mathcal{E}_{j+\frac{1}{2}}^{\varepsilon,-}, j \in \mathbb{Z}$, we assume that

$$
u^{\varepsilon}(x, y) \approx u_{j+\frac{1}{2}}^{\varepsilon}(x, y)=\sum_{k \in \mathbb{N}} \varepsilon^{k} u_{j+\frac{1}{2}}^{(k)}(s)+O\left(\varepsilon^{\infty}\right), \quad s=x-j, \quad(x, y) \in \mathcal{E}_{j+\frac{1}{2}}^{\varepsilon,-} .
$$


In the same way, in the vertical thin slits $\mathcal{E}_{j}^{\varepsilon,-}, j \in \mathbb{Z}$, we assume that

$$
u^{\varepsilon}(x, y) \approx u_{j}^{\varepsilon}(x, y)=\sum_{k \in \mathbb{N}} \varepsilon^{k} u_{j}^{(k)}(y)+O\left(\varepsilon^{\infty}\right), \quad(x, y) \in \mathcal{E}_{j}^{\varepsilon,-} .
$$

With the ansatz (27) and (28), we anticipate that, for small $\varepsilon$, in each slit, the field is essentially a 1D field in the longitudinal variable, except maybe close to the junctions, the transverse variations being contained in the $O\left(\varepsilon^{\infty}\right)$ remainders. The 1D functions appearing in the above expansions are defined on the edges of the half graph $\mathcal{G}^{-}=\mathcal{G} \cap\{y<0\}$. More precisely

$$
u_{j+\frac{1}{2}}^{(k)}: e_{j+\frac{1}{2}}^{-} \equiv[0,1] \mapsto \mathbb{R}, \quad u_{j}^{(k)}: e_{j}^{-} \equiv[-L / 2,0] \mapsto \mathbb{R}
$$

These functions are independent of $\varepsilon$ and given by (24-25) for $k=0$. In what follows, for any $k$, collecting these functions over the index $j$, we define a function $u^{(k)}: \mathcal{G}^{-} \mapsto \mathbb{R}$ (by definition the far field of order $k$ )

$$
u^{(k)} \in H_{b r}^{1}\left(\mathcal{G}^{-}\right), \quad u^{(k)}=u_{j+\frac{1}{2}}^{(k)} \text { on } e_{j+\frac{1}{2}}^{-}, \quad u^{(k)}=u_{j}^{(k)} \text { on } e_{j}^{-},
$$

where $H_{b r}^{1}\left(\mathcal{G}^{-}\right)$is the Hilbert space

$$
H_{b r}^{1}\left(\mathcal{G}^{-}\right)=\left\{u \in L_{2}^{\mu}\left(\mathcal{G}^{-}\right) / u \in H^{1}(e), \forall e \in \mathcal{E} ; \quad \sum_{e \in \mathcal{E}}\|u\|_{H^{1}(e)}^{2}<\infty\right\} .
$$

If we denote $H^{1}\left(\mathcal{G}^{-}\right)=\left\{\left.u\right|_{\mathcal{G}^{-}}, u \in H^{1}(\mathcal{G})\right\}$, we have $H^{1}\left(\mathcal{G}^{-}\right) \subset H_{b r}^{1}\left(\mathcal{G}^{-}\right):$the larger space differs from the smaller one by the fact that we removed the continuity condition (see the definition (6) of $H^{1}(\mathcal{G})$. As we shall see, except for $k=0$, all $u^{(k)}$ will be discontinuous on $\mathcal{G}$.

Substituting (26-27-28) into the eigenvalue problem (21), and separating formally the different powers of $\varepsilon$, we get the following set of problems for the far field terms $u^{(k)}, k \in \mathbb{N}^{*}: \forall j \in \mathbb{Z}$,

$$
\left\{\begin{array}{l}
\partial_{s}^{2} u_{j+\frac{1}{2}}^{(k)}(s)+\lambda^{(0)} u_{j+\frac{1}{2}}^{(k)}(s)=-\sum_{m=0}^{k-1} \lambda^{(k-m)} u_{j+\frac{1}{2}}^{(m)}(s), \quad s \in(0,1) . \\
\partial_{y}^{2} u_{j}^{(k)}(y)+\lambda^{(0)} u_{j}^{(k)}(y)=-\sum_{m=0}^{k-1} \lambda^{(k-m)} u_{j}^{(m)}(y), \quad y \in(-L / 2,0), \quad \partial_{y} u_{j}^{(k)}(0)=0 .
\end{array}\right.
$$

The far field terms $u^{(k)}$ are still not completely defined by (31). More precisely, we need to prescribe transmission conditions at each node $M_{j}$ of the graph $\mathcal{G}$ to link the functions

$$
\left(u_{j+\frac{1}{2}}^{(k)}, u_{j-\frac{1}{2}}^{(k)}, u_{j}^{(k)}\right) .
$$

These transmission conditions will result from the so-called matching conditions. 
- Near field asymptotic expansion: in the neighborhood of the junctions $\mathcal{K}_{j}^{\varepsilon,-}, j \in \mathbb{Z}$, we assume that the following expansion holds

$$
u^{\varepsilon}(x, y) \approx U_{j}^{\varepsilon}(x, y)=\sum_{k \in \mathbb{N}} \varepsilon^{k} U_{j}^{(k)}\left(\frac{x-j}{\varepsilon}, \frac{y+L / 2}{\varepsilon}\right),
$$

where the near field terms $U_{j}^{(k)}$ do not depend on $\varepsilon$ and are defined on "infinite T-shaped junctions":

$$
U_{j}^{(k)}: \mathcal{J}_{j} \mapsto \mathbb{R}, \quad j \in \mathbb{Z},
$$

where the junctions $\mathcal{J}_{j}$ ( all identical for $j \neq 0$ ) are defined by (see also Figure 5)

$$
\mid \begin{aligned}
& \mathcal{J}_{j}=K_{j} \cup B_{j,-} \cup B_{j,+} \cup B_{j, 0}, \quad K_{j}=\left[-\frac{\mu_{j}}{2}, \frac{\mu_{j}}{2}\right] \times[0,1], \\
& B_{j, \pm}= \pm\left(\frac{\mu_{j}}{2},+\infty\right) \times(0,1), \quad B_{j, 0}=\left(-\frac{\mu_{j}}{2}, \frac{\mu_{j}}{2}\right) \times(1,+\infty) .
\end{aligned}
$$
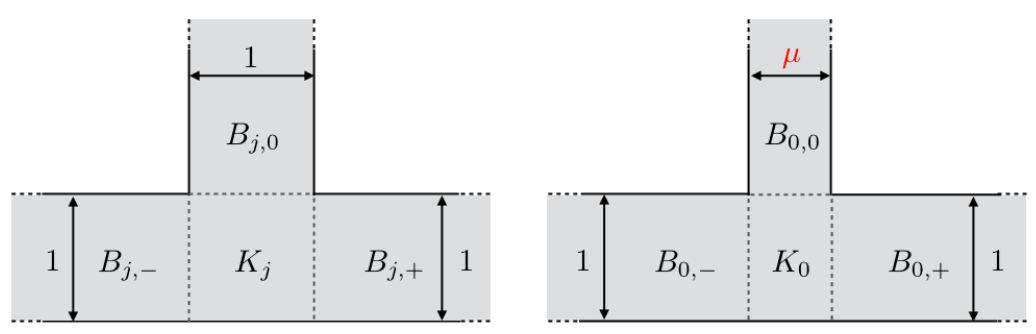

Fig. 5. The junctions $\mathcal{J}_{j}$ (perturbed for $j=0$ (left) and unperturbed for $j \neq 0$ (right))

In what follows, for any $k$, we denote by $U^{(k)}$ (near field of order $k$ ) the set of the near field functions

$$
U^{(k)}:=\left\{U_{j}^{(k)}\right\}_{j \in \mathbb{Z}}
$$

As usual (see [23], [22]), we look for near field terms that belong to

$$
\left\{\begin{array}{l}
U_{j}^{(k)} \in \mathcal{V}_{j}:=\left\{U \in H_{l o c}^{1}\left(\mathcal{J}_{j}\right), \quad w_{j} U \in H^{1}\left(\mathcal{J}_{j}\right)\right\} \text { where } \\
w_{j}(X, Y)=1 \text { in } K_{j}, \quad e^{-\sqrt{ \pm\left(X \mp \mu_{j} / 2\right)}} \text { in } B_{j, \pm}, \quad e^{-\sqrt{Y-1}} \text { in } B_{j, 0} .
\end{array}\right.
$$

which prevents an exponential growth but authorizes a polynomial one. Note that, for $j \neq 0, \mathcal{J}_{j}$ can be identified to $\mathcal{J}_{1}, \mathcal{V}_{j}$ can be identified to $\mathcal{V}_{1}$. Substituting (26-32) into (21) and separating formally the different powers of $\varepsilon$, we find a set of problems for the near field functions $U^{(k)}, k \in \mathbb{N}$ :

$$
\forall j \in \mathbb{Z}, \quad \begin{cases}\Delta U_{j}^{(k)}=-\sum_{m=0}^{k-2} \lambda^{(k-m-2)} U_{j}^{(m)} & \text { in } \mathcal{J}_{j},(i) \\ \partial_{n} U_{j}^{(k)}=0 \text { on } \partial \mathcal{J}_{j} . & (i i)\end{cases}
$$


As for the far field terms, near field terms $U^{(k)}$ are not completely defined by (36) (for instance, any constant function satisfies Problem (36) for $k \leqslant 1$ or more generally any harmonic function that belongs to $\mathcal{V}_{j}$ ). We need to prescribe their behavior at infinity (in the three infinite branches $B_{j, \delta}$, $\delta \in\{+,-, 0\})$, which here again results from the matching conditions.

To make the matching conditions more explicit, we need to describe the form of of the near field terms in the three infinite branches $B_{j, \delta}$ of $\mathcal{J}_{j}$, which relies on a modal expansion. To do so, for any $\delta \in\{0,+,-\}$, we denote by $(\mathfrak{t}, \mathfrak{s})$ the transversal and longitudinal variables of $B_{j, \delta}$, i.e.

$$
\mathfrak{t}=\left\{\begin{array}{ll}
X & \text { in } B_{j, 0}, \\
Y & \text { in } B_{j, \pm},
\end{array} \quad \mathfrak{s}= \begin{cases}Y & \text { in } B_{j, 0} \\
X & \text { in } B_{j, \pm}\end{cases}\right.
$$

and we introduce the two following bases of $L^{2}\left(-\mu_{j} / 2, \mu_{j} / 2\right)$ and $L^{2}(-1 / 2,1 / 2)$ and

$$
\mathbf{v}_{\ell, 0}^{j}(\mathfrak{t}):=\sqrt{\frac{2}{\mu_{j}}} \cos \left(\frac{\ell \pi}{\mu_{j}}\left(\mathfrak{t}+\mu_{j} / 2\right)\right) \quad \text { and } \quad \mathbf{v}_{\ell, \pm}^{j}(\mathfrak{t})=\mathbf{v}_{\ell}(\mathfrak{t}):=\sqrt{2} \cos (\ell \pi \mathfrak{t}),
$$

which are nothing but the eigenfunctions of the corresponding 1D Neumann laplacians.

We begin with some recaps about solution of homogeneous Laplace equations in bands. More precisely, we are interested in the problem

$$
-\Delta u_{j}^{\delta}(\varphi)=0 \quad \text { in } B_{j, \delta}, \quad u_{j}^{\delta}(\varphi)=\varphi \text { on } \Sigma_{j, \delta}, \quad \partial_{n} u_{j}^{\delta}(\varphi)=0 \text { on } \partial B_{j, \delta} \backslash \Sigma_{j, \delta} .
$$

where $\varphi$ is the Dirichlet data belonging to $H^{\frac{1}{2}}\left(\Sigma_{j, \delta}\right)$, with $\delta \in\{0,+,-\}$. It is well-known that this problem has a unique solution in the space

$$
\mathcal{H}^{1}\left(B_{j, \delta}\right):=\left\{v \in H_{l o c}^{1}\left(B_{j, \delta}\right), \frac{v}{\sqrt{1+\mathfrak{s}^{2}}} \in L^{2}\left(B_{j, \delta}\right), \nabla v \in L^{2}\left(B_{j, \delta}\right)\right\} \supset H^{1}\left(B_{j, \delta}\right)
$$

and defining $\mathcal{H a r m}\left(B_{\delta, j}\right):=\left\{U \in \mathcal{H}^{1}\left(B_{\delta, j}\right), \Delta U=0\right.$ in $B_{\delta, j}, \partial_{n} U=0$ on $\left.\partial B_{\delta, j} \backslash \Sigma_{\delta, j}\right\}$,

$$
\text { the mapping } \varphi \mapsto u_{j}^{\delta}(\varphi) \text { is an isomorphism from } H^{\frac{1}{2}}\left(\Sigma_{j, \delta}\right) \text { into the space } \mathcal{H a r m}\left(B_{\delta, j}\right)
$$

The well-posedness of (38) follows from Lax-Milgram's lemma and Hardy's inequality (see for instance [25, Lemma 2.5.7]). Moreover, it can be solved by separation of variables in $(\mathfrak{s}, \mathfrak{t})$, yielding

$$
\left\{\begin{array}{l}
u_{j}^{ \pm}(\varphi)=\sum_{\ell=0}^{+\infty} \beta_{\ell, \pm} e^{-\ell \pi|\mathfrak{s}|} \mathbf{v}_{\ell}(\mathfrak{t}) \quad \text { in } B_{j, \pm}, \beta_{\ell, \pm}=\left(\varphi, \mathbf{v}_{\ell}\right)_{L^{2}\left(\Sigma_{j, \pm}\right)} \\
u_{j}^{0}(\varphi)=\sum_{\ell=0}^{+\infty} \beta_{\ell, 0} e^{-\frac{\ell \pi}{\mu_{j}}|\mathfrak{s}|} \mathbf{v}_{\ell, 0}^{j}(\mathfrak{t}) \quad \text { in } B_{j, 0}, \beta_{\ell, 0}=\left(\varphi, \mathbf{v}_{\ell, 0}^{j}\right)_{L^{2}\left(\Sigma_{j, 0}\right)}
\end{array}\right.
$$

where, we see that $u_{j}^{\delta}$ tends exponentially fast to a constant as $|\mathfrak{s}|$ tends to $+\infty$. We are now ready to give the structure of the near field terms in the bands $B_{j, \delta}$. 
Proposition 2. Assume that there exists a sequence of near fields $\left\{U^{(k)}, k \geqslant 0\right\}$ (see (34)), with 1 $U_{j}^{(k)} \in \mathcal{V}_{j}, \forall j \in \mathbb{Z}$, satisfying (36). Then, for any $k \in \mathbb{N}$, for any $j \in \mathbb{Z}$, there exists $1 D$ polynomials

$$
\left(\hat{U}_{j, \pm}^{(k)}, \hat{U}_{j, 0}^{(k)}\right) \in \mathbb{P}_{k+1} \text { and }\left\{\left(\hat{U}_{j, \ell, \pm}^{(k)}, \hat{U}_{j, \ell, 0}^{(k)}\right) \in \mathbb{P}_{[k / 2]}, \ell \geqslant 1\right\}
$$

such that the function $U_{j}^{(k)}$ admits the following decomposition in the bands $B_{j, \delta}$

$$
\left\{\begin{array}{l}
U_{j}^{(k)}=\hat{U}_{j, \pm}^{(k)}(\mathfrak{s})+\sum_{\ell=1}^{+\infty} \hat{U}_{j, \ell, \pm}^{(k)}(\mathfrak{s}) e^{-\ell \pi|\mathfrak{s}|} \mathbf{v}_{\ell}(\mathfrak{t}) \quad \text { in } B_{j, \pm} \\
U_{j}^{(k)}=\hat{U}_{j, 0}^{(k)}(\mathfrak{s})+\sum_{\ell=1}^{+\infty} \hat{U}_{j, \ell, 0}^{(k)}(\mathfrak{s}) e^{-\frac{\ell \pi}{\mu_{j}}|\mathfrak{s}|} \mathbf{v}_{\ell, 0}^{j}(\mathfrak{t}) \quad \text { in } B_{j, 0}
\end{array}\right.
$$

where the above series converge in $H_{l o c}^{1}\left(B_{j, \delta}\right)$. A more detailed description of $U_{j}^{(k)}$ in each band is

$$
U_{j}^{(k)}=\alpha_{j, \delta}^{(k)} \mathfrak{s}+\mathscr{U}_{j, \delta}^{(k)}(\mathfrak{t}, \mathfrak{s})+U_{j, \delta, \text { part }}^{(k-2)}(\mathfrak{t}, \mathfrak{s}) \text { in } B_{j, \delta}
$$

where $\alpha_{j, \delta}^{(k)}$ is a real constant and

- $\mathscr{U}_{j, \delta}^{(k)}$ belongs to $\mathcal{H a r m}\left(B_{j, \delta}\right)$ (see Def. (40), in particular $\left.\Delta \mathscr{U}_{j, \delta}^{(k)}=0\right)$, thus of the form (see (42))

$$
\left\{\begin{array}{l}
\mathscr{U}_{j, \pm}^{(k)}=\beta_{j, \pm}^{(k)}+\sum_{\ell=1}^{+\infty} \beta_{j, \ell, \pm}^{(k)} e^{-\ell \pi|\mathfrak{s}|} \mathbf{v}_{\ell}(\mathfrak{t}) \quad \text { in } B_{j, \pm} \\
\mathscr{U}_{j, 0}^{(k)}=\beta_{j, 0}^{(k)}+\sum_{\ell=1}^{+\infty} \beta_{j, \ell, 0}^{(k)} e^{-\frac{\ell \pi}{\mu_{j}}|\mathfrak{s}|} \mathbf{v}_{\ell, 0}^{j}(\mathfrak{t}) \quad \text { in } B_{j, 0} .
\end{array}\right.
$$

- $U_{j, \delta, \text { part }}^{(k-2)} \in H_{l o c}^{1}\left(B_{j, \delta}\right)$ is the only particular solution of

$$
\Delta U_{j, \delta, \text { part }}^{(k-2)}=-\sum_{m=0}^{k-2} \lambda^{(k-m-2)} U_{j}^{(m)} \quad \text { in } B_{j, \delta}, \quad \partial_{n} U_{j, \delta, \text { part }}^{(k-2)}=0 \text { on } \partial B_{\delta, j} \backslash \Sigma_{\delta, j}
$$

that admits a decomposition of the form:

$$
\left\{\begin{array}{l}
U_{j, \pm, \text { part }}^{(k-2)}=\hat{U}_{j, \pm, \text { part }}^{(k-2)}(\mathfrak{s})+\sum_{\ell=1}^{+\infty} \hat{U}_{j, \ell, \pm, \text { part }}^{(k-2)}(\mathfrak{s}) e^{-\ell \pi|\mathfrak{s}|} \mathbf{v}_{\ell}(\mathfrak{t}) \\
U_{j, 0, \text { part }}^{(k-2)}=\hat{U}_{j, 0, \text { part }}^{(k-2)}(\mathfrak{s})+\sum_{\ell=1}^{+\infty} \hat{U}_{j, \ell, 0, \text { part }}^{(k-2)}(\mathfrak{s}) e^{-\frac{\ell \pi}{\mu_{j}}|\mathfrak{s}|} \mathbf{v}_{\ell, 0}^{j}(\mathfrak{t}),
\end{array}\right.
$$


with $\left\{\begin{array}{l}\hat{U}_{j, \delta, \text { part }}^{(k-2)} \in \mathbb{P}_{k} \quad \text { and satisfies }(a) \quad \hat{U}_{j, \delta, \text { part }}^{(k-2)}(0)=\partial_{\mathfrak{s}} \hat{U}_{j, \delta, \text { part }}^{(k-2)}(0)=0, \\ \hat{U}_{j, \ell, \delta \text {,part }}^{(k-2)} \in \mathbb{P}_{[k / 2]} \text { and satisfies }(b) \quad \hat{U}_{j, \ell, \delta, \text { part }}^{(k-2)}(0)=0 .\end{array}\right.$

Remark 4. The link between (43) and (44-47) is quite straightforward. In particular,

$$
\hat{U}_{j, \delta}^{(k)}(\mathfrak{s})=\alpha_{j, \delta}^{(k)} \mathfrak{s}+\beta_{j, \delta}^{(k)}+\hat{U}_{j, \delta, \operatorname{part}}^{(k-2)}(\mathfrak{s}), \quad \hat{U}_{j, \ell, \delta}^{(k)}(\mathfrak{s})=\beta_{j, \ell, \delta}^{(k)}+\hat{U}_{j, \ell, \delta, \text { part }}^{(k-2)}(\mathfrak{s})
$$

In the decomposition (44), the label $(k-2)$ for the functions

$$
U_{j, \delta, \text { part }}^{(k-2)}, \hat{U}_{j, \delta, \text { part }}^{(k-2)} \text { or } \hat{U}_{j, \ell, \delta, \text { part }}^{(k-2)}
$$

indicates that they are entirely determined by $\lambda^{(m)}$ and $U_{j}^{(m)}$ for $m \leqslant k-2$.

In opposition, "the harmonic part" of $U_{j}^{(k)}$ in the band $B_{j, \delta}$, namely the function

$$
\alpha_{j, \delta}^{(k)} \mathfrak{s}+\mathscr{U}_{j, \delta}^{(k)}(\mathfrak{t}, \mathfrak{s})
$$

involves infinitely many "free parameters" $\left(\alpha_{j, \delta}^{(k)}, \beta_{j, \delta}^{(k)}\right)$ and $\left\{\beta_{j, \ell, \delta}^{(k)}, \ell \geqslant 1\right\}$. In the following, the decomposition (43) will be used for deriving the matching conditions in Section 2.3. On the other hand, we shall prefer to use the alternate formula (44) for the construction of the terms of the asymptotic expansion (see in particular Section 3).

Remark 5. Integrating (47) with respect to $\mathfrak{t}$ gives

$$
\begin{aligned}
& \int_{0}^{1} U_{j, \pm, \text { part }}^{(k-2)}(\mathfrak{s}, \mathfrak{t}) d \mathfrak{t}=\hat{U}_{j, \pm, \text { part }}^{(k-2)}(\mathfrak{s}) \quad \text { and } \quad \int_{-\frac{\mu_{j}}{2}}^{\frac{\mu_{j}}{2}} U_{j, 0, \text { part }}^{(k-2)}(\mathfrak{s}, \mathfrak{t}) d \mathfrak{t}=\mu_{j} \hat{U}_{j, 0, \text { part }}^{(k-2)}(\mathfrak{s}) . \\
& \text { as well as } \int_{0}^{1} U_{j}^{(k)}(\mathfrak{s}, \mathfrak{t}) d \mathfrak{t}=\hat{U}_{j, \pm}^{(k-2)}(\mathfrak{s}) \quad \text { and } \quad \int_{-\frac{\mu_{j}}{2}}^{\frac{\mu_{j}}{2}} U_{j}^{(k)}(\mathfrak{s}, \mathfrak{t}) d \mathfrak{t}=\mu_{j} \hat{U}_{j, 0}^{(k-2)}(\mathfrak{s}) \text {. }
\end{aligned}
$$

Sketch of the proof of Proposition 2. We only give, for completeness, the main lines of one possible proof for this result. More details can be found for instance in [22, p. 316]. This proof is done by induction on $k$ using separation of variables techniques. We treat here the case of the bands $B_{j, \pm}$. One proceeds similarly in the band $B_{j, 0}$. For each $k$, the near field can be decomposed along the orthonormal basis $\mathbf{v}_{\ell}$. The coefficients of the decomposition are functions of the longitudinal 
variable $\mathfrak{s}$. For $k=0,1$, the announced result simply follows from the fact that any harmonic function in the space $\mathcal{V}_{j}$ admits, in each branch, a decomposition of the form

$$
\alpha_{j, \pm} \mathfrak{s}+\beta_{j, \pm}+\sum_{\ell=1}^{+\infty} \alpha_{j, \ell, \pm} e^{-\ell \pi|\mathfrak{s}|} \mathbf{v}_{\ell}(\mathfrak{t}) \quad \text { in } B_{j, \pm} .
$$

For $k \geqslant 2$, assuming that (43) holds up to $k-1$ and substituting (43) (seen as a particular writing of the modal expansion) into (36), we first see that the coefficient functions $\hat{U}_{j, \pm}^{(k)}(\mathfrak{s})$ must satisfy

$$
\partial_{\mathfrak{s}}^{2} \hat{U}_{j, \pm}^{(k)}=-\sum_{m=0}^{k-2} \lambda^{(m-k-2)} \hat{U}_{j, \pm}^{(m)}
$$

It means that $\hat{U}_{j, \pm}^{(k)}(\mathfrak{s})$ is the sum of an affine function in $\mathfrak{s}$ and a particular solution $\hat{U}_{j, \pm, \text { part }}^{(k-2)}$ of (50), which can be chosen as the one that satisfies the Cauchy conditions (48)(a). It turns out that this particular solution is a polynomial of degree $k$, that has no affine part.

In the same way, one sees that the functions $\hat{U}_{j, \ell, \pm}^{(k)}$ satisfy

$$
\left(\partial_{\mathfrak{s}}^{2} \mp 2 \ell \pi \partial_{\mathfrak{s}}\right) \hat{U}_{j, \ell, \pm}^{(k)}=-\sum_{m=0}^{k-2} \lambda^{(m-k-2)} \hat{U}_{j, \ell, \pm}^{(m)}
$$

The main difference with what preceeds is that a basis of the space of solutions of the homogeneous equation associated with (51) is made of the constant function 1 and the function $e^{2 \ell \pi|\mathfrak{s}|}$. The latter must be eliminated because $U_{j}^{(k)}$ is in $\mathcal{V}_{j}$.

Therefore, $\hat{U}_{j, \ell, \pm}^{(k)}$ is a sum of a constant and a particular solution $\hat{U}_{j, \ell, \pm, \text { part }}^{(k-2)}$ of (51). Taking into account that we exclude exponentially growing functions, we can only impose one condition to "eliminate the constant", by imposing (48)(b). As a matter of fact, as the right hand side of (51) is a polynomial of degree $[k / 2]-1$, it is easily shown that, because $\ell \neq 0$ the particular solution is a polynomial of degree $[k / 2]$.

\subsection{Matching conditions}

\subsubsection{Derivation of the matching conditions}

To find the missing information (the transmission conditions at the vertices of the graph for the far field terms and the behaviour at infinity for the near field terms), we shall write the so-called matching conditions that ensure that far field and near field expansions coincide in some intermediate areas. Indeed, far field and near field expansions are assumed to be both valid in some intermediate areas $\mathcal{M}_{j}^{\varepsilon}$, $j \in \mathbb{Z}$, localized at the left, right and above each junction $K_{j}^{\varepsilon}$,

$$
\mathcal{M}_{j, m}^{\varepsilon}=\mathcal{M}_{j,-}^{\varepsilon} \cup \mathcal{M}_{j,+}^{\varepsilon} \cup \mathcal{M}_{j, 0}^{\varepsilon}
$$
1 
Then the matching zone $\mathcal{M}_{j,+}^{\varepsilon}$ corresponds to $x-j \rightarrow 0$ for the far field and to $X=(x-j) / \varepsilon \rightarrow+\infty \quad 1$ for the near field. Typically, we can choose for instance (see Figure 6 for this example), for any integer 2 $j$, the left and right intermediate areas $\mathcal{M}_{j,-}^{\varepsilon}$ and $\mathcal{M}_{j,+}^{\varepsilon}$ of the form

$$
\mathcal{M}_{j,-}^{\varepsilon}=(0, \varepsilon) \times(j-2 \sqrt{\varepsilon}, j-\sqrt{\varepsilon}), \quad \mathcal{M}_{j,+}^{\varepsilon}=(0, \varepsilon) \times(j+\sqrt{\varepsilon}, j+2 \sqrt{\varepsilon}),
$$

and the vertical intermediate areas of the form

$$
\mathcal{M}_{j, 0}^{\varepsilon}=\left(j-\frac{\mu_{j} \varepsilon}{2}, j+\frac{\mu_{j} \varepsilon}{2}\right) \times\left(-\frac{L}{2}+\sqrt{\varepsilon},-\frac{L}{2}+2 \sqrt{\varepsilon}\right) .
$$

Let us first explain how we proceed in the matching zone $\mathcal{M}_{j,+}^{\varepsilon}$ which involves the junction $\mathcal{J}_{j}$ (for the

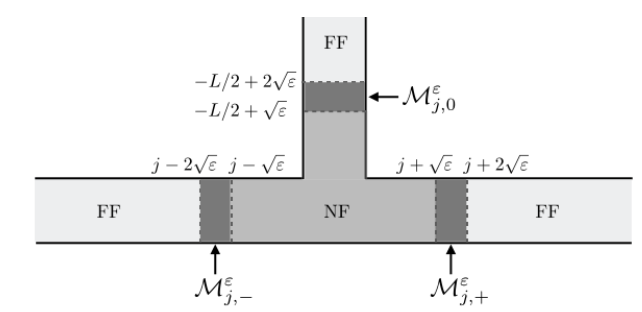

Fig. 6. the matching area $\mathcal{M}_{j, m}^{\varepsilon}=\mathcal{M}_{j,-}^{\varepsilon} \cup \mathcal{M}_{j,+}^{\varepsilon} \cup \mathcal{M}_{j, 0}^{\varepsilon}$ for $j \neq 0$ (NF: near field, FF: far field).

near field) and the edge $e_{j+\frac{1}{2}}$ (for the far field).

We first use the (formal) Taylor series expansion of the far field term $u_{j+\frac{1}{2}}^{(k)}$

$$
u_{j+\frac{1}{2}}^{(k)}(s)=\sum_{\ell \in \mathbb{N}} \partial_{s}^{\ell} u_{j+\frac{1}{2}}^{(k)}(0) \frac{s^{\ell}}{\ell !}
$$

so that the ansatz (27) can be (formally) rewritten as

$$
u_{j+\frac{1}{2}}^{\varepsilon}(x, y)=\sum_{k \in \mathbb{N}} \varepsilon^{k} \sum_{\ell \in \mathbb{N}} \partial_{s}^{\ell} u_{j+\frac{1}{2}}^{(k)}(0) \frac{(x-j)^{\ell}}{\ell !}+O\left(\varepsilon^{\infty}\right), \quad j \in \mathbb{Z} .
$$

On the other hand, using the expansion (43) for $U_{j}^{(k)}$ in $B_{j,+}$, we remark that (32) can be rewritten as

$$
u_{j+\frac{1}{2}}^{\varepsilon}(x, y)=\sum_{k \in \mathbb{N}} \varepsilon^{k} \hat{U}_{j,+}^{(k)}\left(\frac{x-j}{\varepsilon}\right)+O\left(\varepsilon^{\infty}\right), \quad j \in \mathbb{Z} .
$$

where we have noticed that, for $\mathfrak{s}=(x-j) / \varepsilon,(x-j) \in(0,1)$, the terms in factor of $e^{-\ell \pi \mathfrak{s}}, \ell \geqslant 1$ can be put into the $O\left(\varepsilon^{\infty}\right)$ remainder. 
Then, writing $\mathfrak{s}=(x-j) / \varepsilon$, the identification of (52) and (53) leads to relate the two expansions :

$$
\sum_{k \in \mathbb{N}} \sum_{\ell \in \mathbb{N}} \varepsilon^{k+l} \partial_{s}^{\ell} u_{j+\frac{1}{2}}^{(k)}(0) \frac{\mathfrak{s}^{\ell}}{\ell !}=\sum_{k \in \mathbb{N}} \varepsilon^{k} \hat{U}_{j,+}^{(k)}(\mathfrak{s}), \quad j \in \mathbb{Z} .
$$

Finally, identifying the terms of the order $\varepsilon^{k}$ leads to the equalities

$$
\hat{U}_{j,+}^{(k)}(\mathfrak{s})=\sum_{l=0}^{k} \partial_{s}^{\ell} u_{j+\frac{1}{2}}^{(k-\ell)}(0) \frac{\mathfrak{s}^{\ell}}{\ell !}, \quad k \in \mathbb{N}, \quad j \in \mathbb{Z} .
$$

Obviously, we proceed in the same way for the matching areas $\mathcal{M}_{j,-}^{\varepsilon}$ and $\mathcal{M}_{j, 0}^{\varepsilon}$ to obtain

$$
\hat{U}_{j,-}^{(k)}(\mathfrak{s})=\sum_{\ell=0}^{k} \partial_{s}^{\ell} u_{j-\frac{1}{2}}^{(k-\ell)}(1) \frac{\mathfrak{s}^{\ell}}{\ell !}, \quad \text { and } \quad \hat{U}_{j, 0}^{(k)}(\mathfrak{s})=\sum_{\ell=0}^{k} \partial_{y}^{\ell} u_{j}^{(k-\ell)}\left(-\frac{L}{2}\right) \frac{\mathfrak{s}^{\ell}}{\ell !}, \quad k \in \mathbb{N}, \quad j \in \mathbb{Z} .
$$

Using the first equation of (49), the matching conditions $(54,55)$ are equivalent to

$$
\left\{\begin{array}{lll}
\alpha_{j,+}^{(k)}=\partial_{s} u_{j+\frac{1}{2}}^{(k-1)}(0), & \beta_{j,+}^{(k)}=u_{j+\frac{1}{2}}^{(k)}(0), & \hat{U}_{j,+, \text { part }}^{(k-2)}(\mathfrak{s})=\sum_{\ell=2}^{k} \partial_{s}^{\ell} u_{j+\frac{1}{2}}^{(k-\ell)}(0) \frac{\mathfrak{s}^{\ell}}{\ell !} \\
\alpha_{j,-}^{(k)}=\partial_{s} u_{j-\frac{1}{2}}^{(k-1)}(1), & \beta_{j,-}^{(k)}=u_{j-\frac{1}{2}}^{(k)}(1), & \hat{U}_{j,-, \text { part }}^{(k-2)}(\mathfrak{s})=\sum_{\ell=2}^{k} \partial_{s}^{\ell} u_{j-\frac{1}{2}}^{(k-\ell)}(1) \frac{\mathfrak{s}^{\ell}}{\ell !} \\
\alpha_{j, 0}^{(k)}=\partial_{s} u_{j}^{(k-1)}\left(-\frac{L}{2}\right), & \beta_{j,+}^{(k)}=u_{j}^{(k)}\left(-\frac{L}{2}\right), & \hat{U}_{j, 0, \text { part }}^{(k-2)}(\mathfrak{s})=\sum_{\ell=0}^{k} \partial_{s}^{\ell} u_{j}^{(k-\ell)}\left(-\frac{L}{2}\right) \frac{\mathfrak{s}^{\ell}}{\ell !}
\end{array}\right.
$$

\subsubsection{A useful version of the matching conditions}

Let us now anticipate here the way we shall exploit these matching conditions for the construction of the asymptotic expansion by induction on $k$. In fact, these will be used for "building conditions at infinity" for the near field terms in addition to (36). If one assumes $\left(\lambda_{m}, U_{m}^{(k)}\right)$ known for $m \leqslant k-2$, we know from Proposition 2 and Remark 4:

The functions $U_{j, \delta \text {,part }}^{(k-2)}($ in (44)) are known and considered as data.

In view of the decompositions (44)-(45)-(47) of Proposition 2, the equations of the first two columns of (56) can be rewritten as

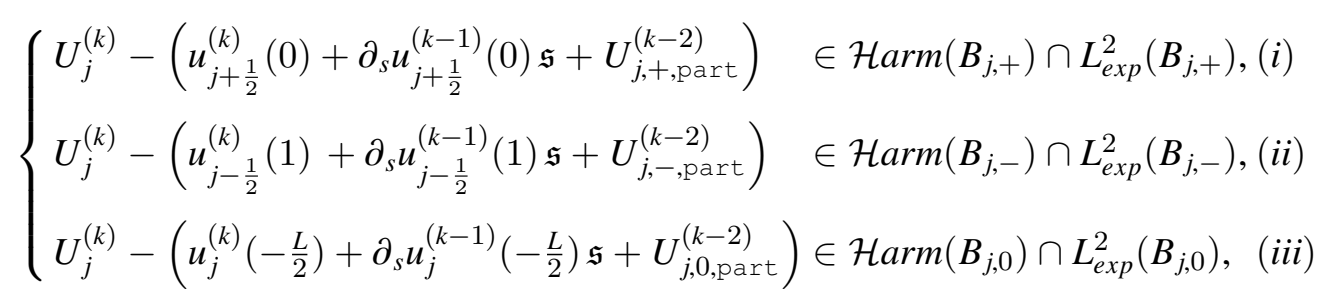


where the spaces $\mathcal{H a r m}\left(B_{j, \delta}\right)$ are defined in (40) and the spaces $L_{\text {exp }}^{2}\left(B_{j, \delta}\right)$ are given by

$$
\left\{\begin{array}{l}
L_{\text {exp }}^{2}\left(B_{j, \pm}\right):=\left\{v \in L_{l o c}^{2}\left(B_{j, \pm}\right), e^{|s|} v \in L^{2}\left(B_{j, \pm}\right)\right\}, \\
L_{\text {exp }}^{2}\left(B_{j, 0}\right)=\left\{v \in L_{l o c}^{2}\left(B_{j, 0}\right), e^{\frac{|s|}{\mu_{j}}} v \in L^{2}\left(B_{j, 0}\right)\right\} .
\end{array}\right.
$$

Note that the functions belonging to $\mathcal{H a r m}\left(B_{j, \delta}\right) \cap L_{\text {exp }}^{2}\left(B_{j, \delta}\right)$ have an expansion of the form (42) with $\beta_{0, \delta}=0$. As a result (see the definitions (37)),

$$
\mathcal{H} \operatorname{arm}\left(B_{j, \delta}\right) \cap L_{\text {exp }}^{2}\left(B_{j, \delta}\right)=\left\{v \in \mathcal{H a r m}\left(B_{j, \delta}\right), \int_{\Sigma_{j, \delta}} v d \sigma=0\right\} .
$$

On the other hand, using Remark 5, the condition of the last column of (56) can be reformulated as

$$
\left\{\begin{array}{l}
\int_{0}^{1} U_{j,+, \text { part }}^{(k-2)}(\mathfrak{s}, \mathfrak{t}) d \mathfrak{t}=\sum_{\ell=2}^{k} \partial_{s}^{\ell} u_{j+\frac{1}{2}}^{(k-\ell)}(0) \frac{\mathfrak{s}^{\ell}}{\ell !}, \\
\int_{0}^{1} U_{j,-, \text { part }}^{(k-2)}(\mathfrak{s}, \mathfrak{t}) d \mathfrak{t}=\sum_{\ell=2}^{k} \partial_{s}^{\ell} u_{j-\frac{1}{2}}^{(k-\ell)}(1) \frac{\mathfrak{s}^{\ell}}{\ell !}, \\
\int_{-\mu_{j} / 2}^{\mu_{j} / 2} U_{j, 0, \text { part }}^{(k-2)}(\mathfrak{s}, \mathfrak{t}) d \mathfrak{t}=\mu_{j} \sum_{\ell=2}^{k} \partial_{s}^{\ell} u_{j}^{(k-\ell)}\left(-\frac{L}{2}\right) \frac{\mathfrak{s}^{\ell}}{\ell !},(i i i)
\end{array}\right.
$$

However, these conditions are redundant, as explained in the following lemma, in the sense that at each step $k$, they appear to be consequences of the matching conditions (58) for $m \leqslant k-1$.

Lemma 2. Assume that (36)-(31) are satisfied (with $m$ instead of $k$ ) for $m \leqslant k$ and that the matching conditions (56) (with $m$ instead of $k$ ) are satisfied for $m \leqslant k-1$. Then, (60) is satisfied.

Proof. The forthcoming proof is done for (60)-(i), but an entirely similar approach leads to (ii)-(iii). As already observed in the proof of Proposition 2 (see (50)), the function $\hat{U}_{j}^{(k)}(\mathfrak{s})$ satisfies

$$
\partial_{\mathfrak{s}} \hat{U}_{j,+}^{(k)}=-\sum_{m=0}^{k-2} \lambda^{(m)} \hat{U}_{j,+}^{(k-2-m)}(\mathfrak{s}) .
$$

But, since the matching conditions (56) are satisfied for $m \leqslant k-1$, we have

$$
\hat{U}_{j,+}^{(k-2-m)}(\mathfrak{s})=\sum_{\ell=0}^{k-2-m} \frac{\mathfrak{s}^{\ell}}{\ell !} \partial_{s}^{\ell} u_{j+\frac{1}{2}}^{(k-2-m-\ell)}(0)
$$

As a result, inverting the roles of $\ell$ and $m$ in the summations, (61) can be rewritten as

$$
\partial_{\mathfrak{s}} \hat{U}_{j,+}^{(k)}=-\sum_{\ell=0}^{k-2} \frac{\mathfrak{s}^{\ell}}{\ell !}\left(\sum_{m=0}^{k-2-\ell} \lambda^{(k)} \partial_{s}^{\ell} u_{j+\frac{1}{2}}^{(k-2-m-\ell)}(0)\right)=\sum_{\ell=0}^{k-2} \frac{\mathfrak{s}^{\ell}}{\ell !} \partial_{s}^{\ell+2} u_{j+\frac{1}{2}}^{(k-2-\ell)}(0),
$$


Here, we have used the far field equations (31) (with $k-2-\ell$ instead of $k$ ) to obtain the second equality. Solving explicitly the previous ordinary differential equation, we obtain (see Proposition 2, Remark 4 and Remark 5 for the notation)

$$
\hat{U}_{j,+}^{(k)}(\mathfrak{s})=\alpha_{j,+}^{(k)} \mathfrak{s}+\beta_{j,+}^{(k)}+\sum_{\ell=0}^{k-2} \frac{\mathfrak{s}^{\ell+2}}{(\ell+2) !} \partial_{s}^{\ell+2} u_{j+\frac{1}{2}}^{(k-2-\ell)}(0)=\alpha_{j,+}^{(k)}+\beta_{j,+}^{(k)} \mathfrak{s}+\sum_{\ell=2}^{k} \frac{\mathfrak{s}^{\ell}}{(\ell) !} \partial_{s}^{\ell} u_{j+\frac{1}{2}}^{(k-\ell)}(0)
$$

Finally, identifying the previous equality with (49) directly gives (60)-(i).

In other words, under the hypotheses of the previous lemma, it suffices to satisfy (58) in order to satisfy the matching conditions (56).

\section{Analysis of far and near field equations}

As usual in the method of asymptotic expansions, the construction of the terms $\left\{\lambda^{(k)}, u^{(k)}, U^{(k)}\right\}$ in the formal expansions will be done by induction on $k$. The idea is, at each step of the induction process to "eliminate" the near field $U^{(k)}$ in order to formulate a problem in $\left\{\lambda^{(k)}, u^{(k)}\right\}$ only, which is similar in its form to the problem (2.2) for $\left\{\lambda^{(0)}, u^{(0)}\right\}$. To do so, in Section 3.1, we first derive transmission conditions on the far fields terms $\left\{u^{(k)}\right\}$ assuming that the whole sequence $\left\{\lambda^{(k)}, u^{(k)}, U^{(k)}\right\}$ exists (Prop. 3). The next two sections prepare the induction process which will be described in Section 4. In Section 3.2, we prove that the above mentioned transmission conditions are also sufficient conditions for the construction of $U^{(k)}$, assuming that the previous terms $\left\{\lambda^{(m)}, u^{(m)}, U^{(m)}\right\}, m<k$ are known as well as the far field term $u^{(k)}$ (Prop. 4). Finally, in Section 3.3, we formulate the problem in $\left(\lambda^{(k)}, u^{(k)}\right)$ and show the existence and uniqueness of the solution provided that $\left\{\lambda^{(m)}, u^{(m)}, U^{(m)}\right\}, m<k$ are known (Prop. 5). The step-by step construction of each term of the asymptotic expansion is conducted in Section 4.

\subsection{Necessary conditions for the existence of the near field terms}

In this section, assuming the existence of the whole sequence $\left\{\lambda^{(k)}, u^{(k)}, U^{(k)}\right\}_{k \in \mathbb{N}}$, we derive non homogeneous transmission conditions for $u^{(k)}$ (similar to those necessarily satisfied by for $u^{(0)}$ ). To obtain these conditions, several approaches are possible. We have chosen the one that consists in "replacing" the problem satisfied by each near field term (namely (36) plus the matching conditions (58)(60)) by an "equivalent" problem set in the bounded domain $K_{j}$ defined in (33) using the so called Dirichlet-toNeumann operators introduced in the next section. Beyond pure theoretical purposes, another interest of this method is that it directly results into a numerical method that will be explained later.

\subsubsection{Dirichlet to Neumann operators}

Let $T_{j}^{\delta} \in \mathscr{L}\left(H^{\frac{1}{2}}\left(\Sigma_{j, \delta}\right), H^{-\frac{1}{2}}\left(\Sigma_{j, \delta}\right)\right)$ be the DtN operator defined by

$$
T_{j}^{ \pm} \varphi=-\partial_{n} u_{j}^{ \pm}(\varphi) \quad \text { on } \Sigma_{j, \pm},
$$


where $u_{j}^{ \pm}(\varphi) \in \mathcal{H}^{1}\left(B_{j, \delta}\right)$ is the unique solution of (38) and $n$ denotes the unit normal vector to $\Sigma_{j, \delta} \quad 1$ outgoing with respect to $K_{j}$. Formula (42) allows us to put the operators $T_{j}^{\delta}$ in diagonal form

$$
T_{j}^{ \pm} \varphi=\sum_{\ell=1}^{+\infty} \ell \pi\left(\varphi, \mathbf{v}_{\ell}\right)_{L^{2}\left(\Sigma_{j, \pm}\right)} \mathbf{v}_{\ell}, \quad T_{j}^{0} \varphi=\sum_{\ell=1}^{+\infty} \frac{\ell \pi}{\mu_{j}}\left(\varphi, \mathbf{v}_{\ell, 0}^{j}\right)_{L^{2}\left(\Sigma_{j, 0}\right)} \mathbf{v}_{\ell, 0}^{j} .
$$

Note that, by definition of the spaces $\mathcal{H a r m}\left(B_{\delta, j}\right)$ and the operators $T_{j}^{\delta}$ (and some abuse of notation),

$$
\forall U \in \mathcal{H a r m}\left(B_{\delta, j}\right), \quad \partial_{n} U+T_{j}^{\delta} U=0 \text { on } \Sigma_{\delta, j} .
$$

\subsubsection{Reduction to a bounded domain}

We wish to characterize, for $j \in \mathbb{Z}$ and $k \in \mathbb{N}$, the restriction of $U_{j}^{(k)}$ to $K_{j}$, denoted $\check{U}_{j}^{(k)}$ for simplicity.

We first introduce the notation: $\Phi_{j}^{(k-2)}:=-\sum_{m=0}^{k-2} \lambda^{(k-m-2)} U_{j}^{(m)}$

where again the superscript $(k-2)$ is here to indicate that this function depends on the $\left(\lambda_{m}, U_{m}\right)$ 's for $m \leqslant k-2$ and is thus a data. Of course, we immediately infer from (36) that

$$
\left\{\begin{array}{l}
\Delta \check{U}_{j}^{(k)}=\Phi_{j}^{(k-2)} \quad \text { in } \mathcal{K}_{j} \\
\partial_{n} \check{U}_{j}^{(k)}=0 \quad \text { on } \partial K_{j} \backslash\left(\Sigma_{j,+} \cup \Sigma_{j,-} \cup \Sigma_{j, 0}\right) .
\end{array}\right.
$$

To close the problem, it remains to derive boundary conditions on the boundaries $\Sigma_{j, \delta}, \delta \in\{0,+,-\}$. More precisely, we write non homogeneous DTN conditions on each boundary $\Sigma_{j, \delta}$. Noticing that constant functions belong to $\mathcal{H a r m}\left(B_{\delta, j}\right)$, we deduce from (58) and the property (64) that

$$
\begin{aligned}
& \partial_{n} U_{j}^{(k)}+T_{j}^{\delta} U_{j}^{(k)}=g_{j, \delta}^{(k-1)} \quad \text { on } \Sigma_{j, \delta}, \quad \text { where } \\
& \left\{\begin{array}{lll}
g_{j,+}^{(k-1)}=\partial_{s} u_{j+\frac{1}{2}}^{(k-1)}(0)+g_{j,+, \text { part }}^{(k-2)}, & g_{j,+, \text { part }}^{(k-2)}=\left(\partial_{n}+T_{j}^{+}\right) U_{j,+, \text { part }}^{(k-2)} & \text { on } \Sigma_{j,+}, \\
g_{j,-}^{(k-1)}=-\partial_{s} u_{j-\frac{1}{2}}^{(k-1)}(1)+g_{j,-, \text { part }}^{(k-2)}, & g_{j,-, \text { part }}^{(k-2)}=\left(\partial_{n}+T_{j}^{-}\right) U_{j,-, \text { part }}^{(k-2)} & \text { on } \Sigma_{j,-,}, \\
g_{j, 0}^{(k-1)}=\partial_{y} u_{j}^{(k-1)}\left(-\frac{L}{2}\right)+g_{j, 0, \text { part }}^{(k-2)}, & g_{j, 0, \text { part }}^{(k-2)}=\left(\partial_{n}+T_{j}^{0}\right) U_{j, 0, \text { part }}^{(k-2)} & \text { on } \Sigma_{j, 0} .
\end{array}\right.
\end{aligned}
$$

Collecting (66) and (67) we see that, in $K_{j}, \check{U}_{j}^{(k)}$ satisfies the boundary value problem

$$
\begin{cases}\Delta \check{U}_{j}^{(k)}=\Phi_{j}^{(k-2)} & \text { in } K_{j}, \\ \partial_{n} \check{U}_{j}^{(k)}+T_{j}^{\delta} \check{U}_{j}^{(k)}=g_{j, \delta}^{(k-1)} & \text { on } \Sigma_{j, \delta}, \quad \delta=0,+,- \\ \partial_{n} \check{U}_{j}^{(k)}=0 & \text { on } \partial K_{j} \backslash\left(\Sigma_{j,+} \cup \Sigma_{j,-} \cup \Sigma_{j, 0}\right) .\end{cases}
$$

Note that, since the image of constant functions by any of the operators $T_{j}^{\delta}$ is 0 , any constant is a solution of the homogeneous boundary value problem corresponding to (69), which means that, at best, a solution 
of (69) is defined up to an additive constant. As a matter of fact, the study of (69) relies on Fredholm's alternative (exactly as the Neumann Laplace problem) and we let the reader prove the following result:

Lemma 3. For $j \in \mathbb{Z}$, let $\Phi \in L^{2}\left(K_{j}\right), g_{\delta} \in H^{-1 / 2}\left(\Sigma_{j, \delta}\right)$. There exists a unique solution $U \in H^{1}\left(K_{j}\right) / \mathbb{R}$ to the problem

$$
\begin{cases}\Delta U=\Phi & \text { in } K_{j}, \\ \partial_{n} U+T_{j}^{\delta} U=g_{\delta} & \text { on } \Sigma_{j, \delta}, \quad \delta=0,+,- \\ \partial_{n} U=0 & \text { on } \partial K_{j} \backslash\left(\Sigma_{j,+} \cup \Sigma_{j,-} \cup \Sigma_{j, 0}\right) .\end{cases}
$$

if and only if the following compatibility condition is satisfied, $\langle\cdot, \cdot\rangle_{\Sigma_{j, \delta}}$ denoting the duality bracket between $H^{-1 / 2}\left(\Sigma_{j, \delta}\right)$ and $H^{1 / 2}\left(\Sigma_{j, \delta}\right)$.

$$
\left\langle g_{+}, 1\right\rangle_{\Sigma_{j,+}}+\left\langle g_{-}, 1\right\rangle_{\Sigma_{j,-}}+\left\langle g_{0}, 1\right\rangle_{\Sigma_{j, 0}}=\int_{K_{j}} \Phi
$$

The fiunction $U$ solution of (70) is omny defined up to an additive constsnt at our disposal.

As we shall see later, in the case of $U_{j}^{(k)}$, the choice of this constant will imposed by the matching conditions.

\subsubsection{Necessary conditions on the far field}

The main result of this section is Proposition 3 whose proof involves two "profile functions" $\mathcal{N}_{j}^{ \pm}$:

- The function $\mathcal{N}_{j}^{-}$is the unique function of $H^{1}\left(K_{j}\right)$ solution to the problem (70) with

$$
\Phi=0, g_{+}=0, g_{-}=1, g_{0}=-1 / \mu_{j} \text { that satisfies } \int_{K_{j}} \mathcal{N}_{j}^{-} d x=0
$$

- The function $\mathcal{N}_{j}^{+}$is the unique function of $H^{1}\left(K_{j}\right)$ solution to the problem (70) with

$$
\Phi=0, g_{+}=-1, g_{-}=0, g_{0}=1 / \mu_{j} \text { that satisfies } \int_{K_{j}} \mathcal{N}_{j}^{+} d x=0
$$

Remark 6. Note that, according to the identification of the junctions $\mathcal{J}_{j}, j \neq 0$ to the single junction $\mathcal{J}_{1}$, all profile functions $\mathcal{N}_{j}^{ \pm}$coincide with $\mathcal{N}_{1}^{ \pm}$. As a consequence, we only have four profile functions for $j=0,1$ and \pm . In addition, $\mathcal{N}_{j}^{+}$and $\mathcal{N}_{j}^{-}$are linked by the following symmetry property:

$$
\mathcal{N}_{j}^{-}(X, Y)=\mathcal{N}_{j}^{+}(-X, Y) \quad \forall j \in \mathbb{N} .
$$

Proposition 3. Assume the existence of a sequence $\left\{\lambda^{(k)}, u^{(k)}, U^{(k)}\right\}$ satisfying (31), (36), (58) and (60). Then, the far field $u^{(k)}$ satisfies for any $k \in \mathbb{N}$ the inhomogeneous generalized Kirchhoff conditions

$$
\partial_{s} u_{j+\frac{1}{2}}^{(k)}(0)-\partial_{s} u_{j-\frac{1}{2}}^{(k)}(1)+\mu_{j} \partial_{y} u_{j}^{(k)}\left(-\frac{L}{2}\right)=\Xi_{j}^{(k-1)},
$$


as well as the inhomogeneous jump conditions

$$
\begin{array}{ll}
u_{j-\frac{1}{2}}^{(k)}(1)-u_{j}^{(k)}\left(-\frac{L}{2}\right)=\Delta_{j,-}^{(k-1)}, & j \in \mathbb{Z},(i) \\
u_{j}^{(k)}\left(-\frac{L}{2}\right)-u_{j+\frac{1}{2}}^{(k)}(0)=\Delta_{j,+}^{(k-1)}, & j \in \mathbb{Z},(i i)
\end{array}
$$

where, $\Phi_{j}^{(k)}, g_{j, \delta}^{(k)}$ and $g_{j, \delta, \text { part }}^{(k)}$ being given by $(65,68)$,

$$
\left\{\begin{aligned}
\Xi_{j}^{(k-1)} & =-\left\langle g_{j,+, \text { part }}^{(k-1)}, 1\right\rangle_{\Sigma_{j,+}}-\left\langle g_{j,-, \text { part }}^{(k-1)}, 1\right\rangle_{\Sigma_{j,-}}-\left\langle g_{j, 0, \text { part }}^{(k-1)}, 1\right\rangle_{\Sigma_{j, 0}}+\int_{K_{j}} \Phi_{j}^{(k-1)} . \\
\Delta_{j,-}^{(k-1)} & =\sum_{\ell=1}^{k}\left(\frac{1}{\ell !} \partial_{y}^{\ell} u_{j}^{(k-\ell)}\left(-\frac{L}{2}\right)-\frac{(-1)^{\ell} \mu_{j}^{\ell}}{2^{\ell} \ell !} \partial_{s}^{\ell} u_{j-\frac{1}{2}}^{(k-\ell)}(1)\right) \\
& +\sum_{\delta \in\{+,-, 0\}}\left\langle g_{j, \delta}^{(k-1)}, \mathcal{N}_{j}^{-}\right\rangle_{\Sigma_{j, \delta}}-\int_{K_{j}} \Phi_{j}^{(k-2)} \mathcal{N}_{j}^{-} . \\
\Delta_{j,+}^{(k-1)}= & \sum_{\ell=1}^{k}\left(\frac{\mu_{j}^{\ell}}{2^{\ell} \ell !} \partial_{s}^{\ell} u_{j+\frac{1}{2}}^{(k-\ell)}(0)-\frac{1}{\ell !} \partial_{y}^{\ell} u_{j}^{(k-\ell)}\left(-\frac{L}{2}\right)\right) \\
& +\sum_{\delta \in\{+,-, 0\}}\left\langle g_{j, \delta}^{(k-1)}, \mathcal{N}_{j}^{+}\right\rangle_{\Sigma_{j, \delta}}-\int_{K_{j}} \Phi_{j}^{(k-2)} \mathcal{N}_{j}^{+}
\end{aligned}\right.
$$

Proof. The result will follow of the investigation of Problem (69) satisfied by $\check{U}_{j}^{(k)}$ and $\check{U}_{j}^{(k+1)}$.

First, we deduce from Lemma 3, that the compatibility condition (71) applied to (69) for $\check{U}_{j}^{(k+1)}$ writes

$$
\left\langle g_{j,+}^{(k)}, 1\right\rangle_{\Sigma_{j,+}}+\left\langle g_{j,-}^{(k)}, 1\right\rangle_{\Sigma_{j,-}}+\left\langle g_{j, 0}^{(k)}, 1\right\rangle_{\Sigma_{j, 0}}=\int_{K_{j}} \Phi_{j}^{(k-1)},
$$

which is easily seen to be equivalent to the condition (75) thanks to (68) and (77)-(i).

Next, to obtain the jump condition (76)(i), we use a particular reciprocity result : let us multiplying equation (69) by $\mathcal{N}_{j}^{-}$and integrate the result over the domain $K_{j}$. Using Green's formula, we get

$$
\sum_{\delta \in\{+,-, 0\}}\left[\left\langle\partial_{n} \mathcal{N}_{j}^{-}, \check{U}_{j}^{(k)}\right\rangle_{\Sigma_{j, \delta}}-\left\langle\partial_{n} \check{U}_{j}^{(k)}, \mathcal{N}_{j}^{-}\right\rangle_{\Sigma_{j, \delta}}\right]=-\int_{K_{j}} \Phi_{j}^{(k-2)} \mathcal{N}_{j}^{-}
$$

which can be rewritten, using the symmetry properties of the DtN operators $T_{j}^{\delta}$

$$
\sum_{\delta \in\{+,-, 0\}}\left[\left\langle\left(\partial_{n}+T_{j}^{\delta}\right) \mathcal{N}_{j}^{-}, \check{U}_{j}^{(k)}\right\rangle_{\Sigma_{j, \delta}}-\left\langle\left(\partial_{n}+T_{j}^{\delta}\right) \check{U}_{j}^{(k)}, \mathcal{N}_{j}^{-}\right\rangle_{\Sigma_{j, \delta}}\right]=-\int_{K_{j}} \Phi_{j}^{(k-2)} \mathcal{N}_{j}^{-}
$$


Using the DtN boundary conditions satisfied by $\check{U}_{j}^{(k)}$ (cf. (69)) and $\mathcal{N}_{j}^{-}$(see (70) and (72)), we get

$$
\int_{\Sigma_{j,-}} \check{U}_{j}^{(k)}-\frac{1}{\mu_{j}} \int_{\Sigma_{j, 0}} \check{U}_{j}^{(k)}-\sum_{\delta \in\{+,-, 0\}}\left\langle g_{j, \delta}^{(k-1)}, \mathcal{N}_{j}^{-}\right\rangle_{\Sigma_{j, \delta}}=-\int_{K_{j}} \Phi_{j}^{(k-2)} \mathcal{N}_{j}^{-} .
$$

Finally, substituting (60) into (79) leads, after some easy computations, to the jump condition (76)-(i).

Proceeding in the same manner with $\mathcal{N}_{j}^{+}$instead of $\mathcal{N}_{j}^{-}$leads to the jump condition (76)-(ii).

\subsection{Towards the inductive construction of the near field terms}

In this section, we assume that we are inside an induction process and wish to construct $U^{(k)}$ for $k \geqslant 1$ assuming that

$\left(H_{k}\right) \quad\left\{\begin{array}{l}\text { The far field terms }\left(u^{(m)}, \lambda^{(m)}\right), m \leqslant k \text { and the near field terms } U^{(m)}, m \leqslant k-1 \text { have been } \\ \text { constructed so as to satisfy }(31),(75) \text { and (76) on the one hand, (36), (56) on the other hand. }\end{array}\right.$

Proposition 4. Assume that $\left(H_{k}\right)$ holds. Then, for each $j$, there exists a unique near field term $U_{j}^{(k)} \in \mathcal{V}_{j}$ satisfying (36) and (58).

Proof. We are going to construct the solution piecewise as

$$
U_{j}^{(k)}=\check{U}_{j}^{(k)} \text { in } K_{j}, \quad U_{j}^{(k)}=U_{j, \delta}^{(k)} \text { in } B_{j, \delta}, \quad \delta= \pm, 0, \quad \text { according to the following lines; }
$$

(1) We build $\check{U}_{j}^{(k)}$ in order that it satisfies the non homogeneous Laplace equation (69) in $K_{j}$.

(2) We construct $U_{j, \delta}^{(k)}$ in $B_{j, \delta}, \delta \in\{ \pm, 0\}$ by extending $\check{U}_{j}^{(k)}$.

(3) We check that $U_{j}^{(k)}$ satisfies the matching conditions (58).

Step 1. First, according to (69), we construct $\check{U}_{j}^{(k)}$ as a solution of

$$
\begin{cases}\Delta \check{U}_{j}^{(k)}=\Phi_{j}^{(k-2)} & \text { in } K_{j}, \\ \partial_{n} \check{U}_{j}^{(k)}+T_{j}^{\delta} \check{U}_{j}^{(k)}=g_{j, \delta}^{(k-1)} & \text { on } \Sigma_{j, \delta}, \quad \delta=0, \pm, \\ \partial_{n} \check{U}_{j}^{(k)}=0 & \text { on } \partial K_{j} \backslash\left(\Sigma_{j, \pm} \cup \Sigma_{j, 0}\right) .\end{cases}
$$

which, in view of Lemma 3, is well-posed in $H^{1}\left(K_{j}\right) / \mathbb{R}$ (note that due to $\left(H_{k}\right), \Phi_{j}^{(k-2)}$ and $g_{j, \delta}^{(k-1)}$ are known). Indeed, as explained in Section 3.1.3 (proof of Proposition 3), the condition (75) (written for $k-1$ instead of $k)$ is a consequence of $\left(H_{k}\right)$ and is nothing but the compatibility condition for the wellposedness of (80). However, as this solution is defined up to an additive constant, we need to adjust this constant appropriately. 
This is where we use the fact that we want $U_{j}^{(k)}$ to satisfy also (58), which implies, as shown below,

$$
\left\{\begin{array}{l}
\int_{\Sigma_{j,+}} U_{j}^{(k)}=\sum_{\ell=0}^{k} \frac{\mu_{j}^{\ell}}{2^{\ell} \ell !} \partial_{s}^{\ell} u_{j+\frac{1}{2}}^{(k-\ell)}(0), \\
\int_{\Sigma_{j,-}} U_{j}^{(k)}=\sum_{\ell=0}^{k} \frac{(-1)^{\ell} \mu_{j}^{\ell}}{2^{\ell} \ell !} \partial_{s}^{\ell} u_{j-\frac{1}{2}}^{(k-\ell)}(1), \\
\int_{\Sigma_{j, 0}} U_{j}^{(k)}=\mu_{j} \sum_{\ell=0}^{k} \frac{1}{\ell !} \partial_{y}^{\ell} u_{j}^{(k-\ell)}\left(-\frac{L}{2}\right),
\end{array}\right.
$$

Indeed, let us prove (81)-(i) (the proof is the same for the other two equalities). Using the mean value property (59) for functions in $\mathcal{H a r m}\left(B_{j, \delta}\right) \cap L_{\text {exp }}^{2}\left(B_{j, \delta}\right)$, (58)-(i) implies

$$
\int_{\Sigma_{j,+}} U_{j}^{(k)}-\left(u_{j+\frac{1}{2}}^{(k)}(0)+\partial_{s} u_{j+\frac{1}{2}}^{(k-1)}(0) \mathfrak{s}+U_{j,+, \text { part }}^{(k-2)}\right)=0 .
$$

However, Lemma 2, which we can apply thanks to $\left(H_{k}\right)$, implies (60)-(i). It is then easy to verify that the previous equality is nothing but (81)-(i).

A priori, imposing each of the equalities in (81) would lead to fix the missing constant. Fortunately, there is no possible ambiguity thanks to the compatibility jump conditions (76):

Lemma 4. Assume that the conditions (76) hold. Then, if $\check{U}_{j}^{(k)}$ is a solution of (80), the three equalities (81) (written with $\check{U}_{j}^{(k)}$ instead of $U_{j}^{(k)}$ ) are equivalent.

Proof. For simplicity, let us adopt the notation

$$
\mathcal{L}(\mathcal{N}):=\sum_{\delta \in\{+,-, 0\}}\left\langle g_{j, \delta}^{(k-1)}, \mathcal{N}\right\rangle_{\Sigma_{j, \delta}}-\int_{K_{j}} \Phi_{j}^{(k-2)} \mathcal{N} .
$$

so that the jump condition (76)-(i), which is a consequence of $\left(H_{k}\right)$, can be rearranged as

$$
\sum_{\ell=0}^{k} \frac{(-1)^{\ell} \mu_{j}^{\ell}}{2^{\ell} \ell !} \partial_{s}^{\ell} u_{j-\frac{1}{2}}^{(k-\ell)}(1)=\sum_{\ell=0}^{k} \frac{1}{\ell !} \partial_{y}^{\ell} u_{j}^{(k-\ell)}\left(-\frac{L}{2}\right)+\mathcal{L}\left(\mathcal{N}_{j}^{-}\right)
$$

On the other hand, proceeding as we did to obtain (79), we deduce from (80) that

$$
\mathcal{L}\left(\mathcal{N}_{j}^{-}\right)=\int_{\Sigma_{j,-}} \check{U}_{j}^{(k)}-\frac{1}{\mu_{j}} \int_{\Sigma_{j, 0}} \check{U}_{j}^{(k)}
$$


so that (82) can be rewritten as

$$
\sum_{\ell=0}^{k} \frac{(-1)^{\ell} \mu_{j}^{\ell}}{2^{\ell} \ell !} \partial_{s}^{\ell} u_{j-\frac{1}{2}}^{(k-\ell)}(1)-\int_{\Sigma_{j,-}} \check{U}_{j}^{(k)}=\sum_{\ell=0}^{k} \frac{1}{\ell !} \partial_{y}^{\ell} u_{j}^{(k-\ell)}\left(-\frac{L}{2}\right)-\frac{1}{\mu_{j}} \int_{\Sigma_{j, 0}} \check{U}_{j}^{(k)}
$$

which shows the equivalence between (81)-(i) and (81)-(iii). In the same way, the jump condition (76)(ii) implies the equivalence between (81)-(ii) and (81)-(iii).

As a consequence of the lemma, we fix the free constant in order that each of the conditions (81) (with $\check{U}_{j}^{(k)}$ instead of $U_{j}^{(k)}$ ) is satisfied.

Step 2. Next, we construct the extensions $U_{j, \delta}^{(k)}$ of $\check{U}_{j}^{(k)}$ in the bands $B_{j, \delta}(\delta \in\{0,+,-\})$. Below, we restrict ourselves to the case of $B_{j,+}$, the other bands being treated in the same way.

In $B_{j,+}$, according to the desired matching condition (58)- $(i)$, we need to take $U_{j,+}^{(k)}$ in the form

$$
U_{j,+}^{(k)}=\partial_{s} u_{j+\frac{1}{2}}^{(k-1)}(0) \mathfrak{s}+u_{j+\frac{1}{2}}^{(k)}(0)+U_{j,+, \text { part }}^{(k-2)}+\mathscr{V}_{j,+}^{(k)}
$$

where $\mathscr{V}_{j,+}^{(k)}$ should belong to the space $\mathcal{H a r m}\left(B_{j,+}\right) \cap L_{\text {exp }}^{2}\left(B_{j,+}\right)$. As an element of $\mathcal{H a r m}\left(B_{j,+}\right)$, according to (41),we have necessarily

$$
\mathscr{V}_{j,+}^{(k)}=u_{j}^{+}\left(\varphi_{j,+}^{(k)}\right) \quad \text { for some } \varphi_{j,+}^{(k)} \in H^{\frac{1}{2}}\left(\Sigma_{j,+}\right)
$$

At this stage it only remains to choose $\varphi_{j,+}^{(k)}$. In order to match the values of $\check{U}_{j}^{(k)}$ and $U_{j,+}^{(k)}$ on $\Sigma_{j,+}, \quad \begin{aligned} & 27 \\ & 28\end{aligned}$ according to (83), we need to choose

$$
\varphi_{j,+}^{(k)}=\left.\check{U}_{j}^{(k)}\right|_{\Sigma_{j,+}}-\left(\frac{\mu_{j}}{2} \partial_{s} u_{j+\frac{1}{2}}^{(k-1)}(0)+u_{j+\frac{1}{2}}^{(k)}(0)+\left.U_{j,+, \text { part }}^{(k-2)}\right|_{\Sigma_{j,+}}\right)
$$

This choice is in fact sufficient to ensure also the matching of the normal derivatives. In view of the DtN boundary condition satisfied by $\check{U}_{j}^{(k)}$, it suffices to check that

$$
\left(\partial_{n}+T_{j,+}\right) U_{j,+}^{(k)}=g_{j,+}^{(k-1)}
$$

But, using (83) and (64) for $\mathscr{V}_{j,+}^{(k)}$, this is nothing but the definition of $g_{j,+}^{(k-1)}$ (see Section 3.1.2).

Step 3. It remains to check that $U_{j}^{(k)}$ satisfies the matching conditions (58). In view of Lemma 2, it suffices to verify that (58)-( $i$ ) is satisfied, i.e.

$$
\mathscr{V}_{j,+}^{(k)} \in \mathcal{H} \operatorname{arm}\left(B_{j,+}\right) \cap L_{\text {exp }}^{2}\left(B_{j,+}\right)
$$


According to the characterization (59), this simply amounts to verifying that $\varphi_{j,+}^{(k)}$ has mean value 0 along $\Sigma_{j,+}$. However, thanks to Lemma 2, (81) rewrites as

$$
\int_{\Sigma_{j,+}} U_{j}^{(k)}-\left(\frac{\mu_{j}}{2} \partial_{s} u_{j+\frac{1}{2}}^{(k-1)}(0)+u_{j+\frac{1}{2}}^{(k)}(0)+\int_{\Sigma_{j,+}} U_{j,+, \text { part }}^{(k-2)}\right)=0
$$

which, using again Lemma 2, is nothing but $\int_{\Sigma_{j,+}} \varphi_{j,+}^{(k)}=0$ (see (84)).

3.3. The problem in $\left(u^{(k)}, \lambda^{(k)}\right)$.

Here we assume that $\left\{\lambda^{(m)}, u^{(m)}, U^{(m)}\right\}$ are known for $m<k$. Collecting the results of the previous sections (far field equations (31), Kirchhoff conditions (75), and non-homogeneous jump conditions (76)), we see that $\left(u^{(k)}, \lambda^{(k)}\right)$ should satisfy the following problem:

$$
\forall j \in \mathbb{Z}, \quad\left\{\begin{array}{l}
\partial_{s}^{2} u_{j+\frac{1}{2}}^{(k)}(s)+\lambda^{(0)} u_{j+\frac{1}{2}}^{(k)}(s)=-\lambda^{(k)} u_{j+\frac{1}{2}}^{(0)}(s)-f_{j+\frac{1}{2}}^{(k-1)}(s), \quad s \in(0,1), \\
\partial_{y}^{2} u_{j}^{(k)}(y)+\lambda^{(0)} u_{j}^{(k)}(y)=-\lambda^{(k)} u_{j}^{(0)}(y)-f_{j}^{(k-1)}(y), \\
\partial_{y} u_{j}^{(k)}(0)=0, \\
\partial_{s} u_{j+\frac{1}{2}}^{(k)}(0)-\partial_{s} u_{j-\frac{1}{2}}^{(k)}(1)+\mu_{j} \partial_{y} u_{j}^{(k)}\left(-\frac{L}{2}\right)=\Xi_{j}^{(k-1)}, \\
u_{j-\frac{1}{2}}^{(k)}(1)-u_{j}^{(k)}\left(-\frac{L}{2}\right)=\Delta_{j,-}^{(k-1)}, \quad u_{j}^{(k)}\left(-\frac{L}{2}\right)-u_{j+\frac{1}{2}}^{(k)}(0)=\Delta_{j,+}^{(k-1)} .
\end{array}\right.
$$

where the data at the right hand sides of last two equations are given by (77) and (68), and thus known from $\left\{\lambda^{(m)}, u^{(m)}, U^{(m)}\right\}, m<k$, while

$$
f_{j+\frac{1}{2}}^{(k-1)}:=\sum_{m=1}^{k-1} \lambda^{(k-m)} u_{j+\frac{1}{2}}^{(m)}, \quad f_{j}^{(k-1)}:=\sum_{m=1}^{k-1} \lambda^{(k-m)} u_{j}^{(m)}, \quad j \in \mathbb{Z} .
$$

Let $E\left(\lambda_{0}\right):=\operatorname{span}\left[u^{(0)}\right] \subset H^{1}\left(\mathcal{G}^{-}\right) \subset H_{b r}^{1}\left(\mathcal{G}^{-}\right)$be the eigenspace of $\mathcal{A}_{s}^{\mu}$ associated to the eigenvalue $\lambda_{0}$.

Proposition 5. Let $k \geqslant 1$. Assume that

$$
f^{(k-1)} \in L_{2}^{\mu}\left(\mathcal{G}^{-}\right),\left\{\Xi_{j}^{(k-1)}\right\}_{j \in \mathbb{Z}} \in l_{2}(\mathbb{Z}) \text { and }\left\{\Delta_{j, \pm}^{(k-1)}\right\}_{j \in \mathbb{Z}} \in l_{2}(\mathbb{Z})
$$

then there exists a unique $\left(u^{(k)}, \lambda^{(k)}\right) \in H_{b r}^{1}\left(\mathcal{G}^{-}\right) / E\left(\lambda_{0}\right) \times \mathbb{R}$ solution of (85). Moreover

$$
\begin{gathered}
\lambda^{(k)}=\left\|u^{(0)}\right\|_{L_{2}^{\mu}\left(\mathcal{G}^{-}\right)}^{-2}\left(2 \sum_{j \in \mathbb{Z}} \widetilde{\Xi}_{j}^{(k-1)} \boldsymbol{u}_{j}^{(0)}-\left(f^{(k-1)}, u^{(0)}\right)_{L_{2}^{\mu}\left(\mathcal{G}^{-}\right)}\right), \\
\text {where } \quad \widetilde{\Xi}_{j}^{(k-1)}=\Xi_{j}^{(k-1)}-\frac{\sqrt{\lambda^{(0)}}}{\sin \sqrt{\lambda^{(0)}}}\left(\Delta_{j+1,-}^{(k-1)}-\Delta_{j-1,+}^{(k-1)}+\cos \sqrt{\lambda^{(0)}}\left(\Delta_{j,+}^{(k-1)}-\Delta_{j,-}^{(k-1)}\right)\right) .
\end{gathered}
$$


Proof. The proof of this theorem is an elementary application of the Fredholm's alternative. A similar proof can be found, for instance in [26] (see Theorem 4.10, Corollary 2.2 and Theorem 2.13). Note that (88) is the necessary compatibility condition for the existence of a solution of (85) seen as a generalized boundary value problem for $u^{(k)}$.

Remark 7. In the solution of (85), the field $u^{(k)}$ is defined up to the addition of any element of $E\left(\lambda_{0}\right)$. The uniqueness can be restored by imposing, for instance, any linear condition of the form $\ell\left(u^{k}\right)=0$, where $\ell$ is a linear form of $H_{b r}^{1}\left(\mathcal{G}^{-}\right)$such that $\ell\left(u^{0}\right) \neq 0$. The choice of $\ell$ will specify the construction of a particular pseudo-mode. In Section 4 (see e.g. (115)), we shall precise our choice of $\ell$.

Besides, we can notice that the expression of $\lambda^{(k)}$ given by (88) is homogeneous of degree 0 with respect to the eigenvector $u^{(0)}$ so that one can check that it does not depend on its normalization. Indeed, it can be shown by induction that $f_{j}^{(k-1)}$ and $\widetilde{\Xi}_{j}^{(k-1)}$ depend linearly on $u^{(0)}$.

We conclude this section by a symmetry property (in the variable $x$ ) of the far field $u^{(k)}$.

Lemma 5. Let $k \in \mathbb{N}^{*}$. Assume that (87) holds and that condition (88) is fulfilled. Suppose also that the following symmetry conditions hold: $\forall j \in \mathbb{Z}$,

$$
(i) \quad\left\{\begin{array} { l } 
{ f _ { - j - \frac { 1 } { 2 } } ^ { ( k - 1 ) } ( 1 - s ) = f _ { j + \frac { 1 } { 2 } } ^ { ( k - 1 ) } ( s ) s \in [ 0 , 1 ] } \\
{ f _ { - j } ^ { ( k - 1 ) } ( y ) = f _ { j } ^ { ( k - 1 ) } ( y ) \quad y \in [ - \frac { L } { 2 } , 0 ] }
\end{array} \quad ( i i ) \quad \left\{\begin{array}{c}
\Delta_{j,+}^{(k-1)}=-\Delta_{-j,-}^{(k-1)} \\
\Xi_{j}^{(k-1)}=\Xi_{-j}^{(k-1)} .
\end{array}\right.\right.
$$

Then, the solution $u^{(k)}$ of Problem (85) is symmetric, i.e., for any $j \in \mathbb{Z}$,

$$
\left\{\begin{array}{l}
u_{-j-\frac{1}{2}}^{(k)}(1-s)=u_{j+\frac{1}{2}}^{(k)}(s) s \in[0,1] \\
u_{-j}^{(k)}(y)=u_{j}^{(k)}(y) \quad y \in\left[-\frac{L}{2}, 0\right]
\end{array}\right.
$$

Proof. Let us introduce the function $\hat{u}^{(k)}$ defined by

$$
\hat{u}_{j+\frac{1}{2}}^{(k)}(s)=u_{-j-\frac{1}{2}}^{(k)}(1-s), \quad s \in[0,1], \quad \hat{u}_{j}^{(k)}(y)=u_{-j}^{(k)}(y), \quad y \in\left[-\frac{L}{2}, 0\right] .
$$

Then, the function $w^{(k)}=\hat{u}^{(k)}-u^{(k)}$ solves the homogeneous problem (23). Consequently, there exists a real constant $c$ such that $w^{(k)}=c u^{(0)}$.

Since $w_{1 / 2}^{(k)}(0)=u_{-1 / 2}^{(k)}(1)-u_{1 / 2}^{(k)}(0)=\Delta_{0,-}^{(k-1)}+\Delta_{0,+}^{(k-1)}=0$, we deduce that $c=0$ and $w^{(k)}=0$.

\section{The asymptotic expansion: existence and algorithm}

By repeating applications of Proposition 5 and Proposition 4 (successively), we are able to define a recursive procedure to construct all the terms of the different asymptotic expansions (far field expansion, near field expansion and eigenvalue expansion) up to any order. The construction is done by induction. 
Moreover, we can derive explicit formulas for the far field terms and semi-explicit expressions for the near field terms, which are suitable for the numerical computations of the successive terms of the asymptotic expansion. In particular, we point out two important features of the forthcoming construction:

(1) First, by induction, all far field terms $\left(u_{j+1 / 2}^{(k)}, u_{j}^{(k)}\right)$ inherit of the symmetry property (91). Moreover, the near field term satisfy an analogous symmetry property

$$
U_{-j}^{(k)}(X, Y)=U_{j}^{(k)}(-X, Y) \quad \forall j \in \mathbb{N} .
$$

In practice, at each step $k$, it is consequently sufficient to compute $u_{j+1 / 2}^{(k)}, u_{j}^{(k)}$ and $U_{j}^{(k)}$ for $j \in \mathbb{N}$.

(2) Secondly, an explicit dependance with respect to $j$ can be proved by induction. This turns out to be very useful from the numerical point of view.

This section is organized as follows. First, we initialize the induction process for $k=0$ in Section 4.1. Then, we proceed to the first induction step $k=1$ in Section 4.2. This step has a pedagogical interest for the understanding of the general induction step at any order $k$ made in Section 4.3.

\subsection{Order 0 : initialization of the algorithm}

We start from an eigenvalue $\lambda^{(0)}$ of the operator $\mathcal{A}_{s}^{\mu}$ defined in the limit graph and the associated symmetric eigenvector $u^{(0)}$ given by (24-25).

For convenience in the forthcoming exposition, we shall use the following alternative expressions of $\left(u_{j+\frac{1}{2}}^{(0)}, u_{j}^{(0)}\right)$ for $j \geqslant 0$, (completed by the symmetry property (91))

$$
\begin{cases}u_{j+\frac{1}{2}}^{(0)}(s)=r^{j}\left(a_{0}^{(0)} \cos \left(\sqrt{\lambda^{(0)}} s\right)+b_{0}^{(0)} \sin \left(\sqrt{\lambda^{(0)}} s\right)\right), & s=x-j \in[0,1], \\ u_{j}^{(0)}(y)=r^{j} c_{0}^{(0)} \cos \left(\sqrt{\lambda^{(0)}} y\right), & y \in[-L / 2,0] .\end{cases}
$$

where $a_{0}^{(0)}=1, \quad b_{0}^{(0)}=\sin \left(\sqrt{\lambda^{(0)}}\right)^{-1}\left(\left(r-\cos \left(\sqrt{\lambda^{(0)}}\right)\right), \quad c_{0}^{(0)}=\cos \left(\sqrt{\lambda^{(0)}} L / 2\right)\right)^{-1}$.

According to Section 3 and more particularly Proposition 4, each near field terms $U_{j}^{(0)}, j \in \mathbb{Z}$ is then defined as the unique solution of $(36,58)$ for $k=0$. Indeed, thanks to the convention of Remark 3 , it is easy to check that Assumption $\left(H_{0}\right)$ (needed for applying Proposition 4 ) is nothing but the fact that $u^{(0)}$ is an eigenvector associated to the eigenvalue $\lambda^{(0)}$, which is precisely our starting point.

Moreover, since $\Phi^{(-1)}=0$, it is then easy to see that for all $j, U_{j}^{(0)}$ is constant equal to $u_{j+\frac{1}{2}}^{(0)}(0)$, i. e.

$$
U_{j}^{(0)}=r^{|j|}, \quad \forall j \in \mathbb{Z} .
$$

\subsection{Order 1 : first induction step}

We shall construct in turn

1

2

3

4

5 
(1) the coefficient $\lambda^{(1)}$ and the far field terms $u^{(1)}$ by means of an explicit resolution (see Remark 8) of (85) for $k=1$. We prove that $u^{(1)}$ is symmetric (in the sense of (91)) and that

$$
\begin{cases}u_{j+\frac{1}{2}}^{(1)}(s)=r^{j} \sum_{\ell=0}^{1} s^{\ell}\left(a_{\ell}^{(1)}(j) \cos \left(\sqrt{\lambda_{0}} s\right)+b_{\ell}^{(1)}(j) \sin \left(\sqrt{\lambda_{0}} s\right)\right), s \in[0,1], \quad j \in \mathbb{N}, \\ u_{j}^{(1)}(y)=r^{j} \sum_{\ell=0}^{1} y^{\ell}\left(c_{\ell}^{(1)}(j) \cos \left(\sqrt{\lambda_{0}} y\right)+d_{\ell}^{(1)}(j) \sin \left(\sqrt{\lambda_{0}} y\right)\right), & y \in\left[-\frac{L}{2}, 0\right], \quad j \in \mathbb{N}^{*}, \\ u_{0}^{(1)}(y)=\sum_{\ell=0}^{1} y^{\ell}\left(c_{\ell, 0}^{(1)} \cos \left(\sqrt{\lambda_{0}} y\right)+d_{\ell, 0}^{(1)} \sin \left(\sqrt{\lambda_{0}} y\right)\right), & y \in\left[-\frac{L}{2}, 0\right] .\end{cases}
$$

where the coefficients $\left(a_{\ell}^{(1)}(j), d_{\ell}^{(1)}(j), c_{\ell}^{(1)}(j), d_{\ell}^{(1)}(j), c_{\ell, 0}^{(1)}, d_{\ell, 0}^{(1)}\right)$ are explicitly determined.

(2) the near field term $U^{(1)}$, which is symmetric in the sense of (92) and is of the form

$$
U_{j}^{(1)}(\cdot)=r^{j}\left(\mathcal{U}^{(1)}(\cdot)+\mathcal{P}^{(1)}(j)\right) \quad \forall j \in \mathbb{N}^{*}, \quad \text { with } \mathcal{J}_{j} \text { identified to } \mathcal{J}_{1}
$$

where $\mathcal{U}^{(1)} \in H_{\text {loc }}^{1}\left(\mathcal{J}_{1}\right)$ is a so-called profile function and the constant $\mathcal{P}^{(1)}(j)$ is a polynomial of degree 1 in $j$. Note that (96) avoids the case $j=0$ which will be the object of a separate treatment.

Remark 8. To avoid a boring exposition of long and complicated formulas or expressions, we shall most often restrict ourselves to explain how these explicit computations can be done, without giving the results (this will be also the case in Section 4.3). Note however that these formulas are necessary and used in the numerical method presented in Section 6, while the general form of these formulas will be used for the error analysis of Section 5.

Remark 9. Note that it is natural that the vertical edge corresponding to $j=0$ is treated in a separate manner since it corresponds to the refined branch ot the thich graph $\Omega_{\varepsilon}^{\mu,-}$.

4.2.1. Determination of $\lambda^{(1)}$ and $u^{(1)}$

They are obtained by solving (85) for $k=1$. Let us investigate the structure of the data

$$
\left(f_{j+1 / 2}^{(0)}, f_{j}^{(0)}, \Delta_{j,+}^{(0)}, \Delta_{j,-}^{(0)}, \Xi_{j}^{(0)}\right)
$$

of this problem. First of all, by definition, we know that

$$
\forall j \in \mathbb{Z}, \quad f_{j+1 / 2}^{(0)}=f_{j}^{(0)}=0 .
$$

Concerning $\Delta_{j, \pm}^{(0)}$, we first notice that, from (68), (93) and Remarks 3 and 6,

$$
\forall j \geqslant 0, \quad g_{j, \delta}^{(0)}=r^{j} g_{\delta}^{(0)}, \quad \text { and } \quad \forall j \in \mathbb{Z}, \quad g_{-j, 0}^{(0)}=g_{j, 0}^{(0)} \quad g_{-j,+}^{(0)}=g_{j,-}^{(0)}
$$

where moreover

$$
g_{+}^{(0)}=-g_{-}^{(0)}=b_{0}^{(0)} \sqrt{\lambda^{(0)}}, \quad g_{0}^{(0)}=c_{0}^{(0)} \sqrt{\lambda^{(0)}} \sin \left(\sqrt{\lambda^{(0)}} L / 2\right) .
$$


Using the definition (77)(ii) and (iii) for $\Delta_{j, \pm}^{(0)}$, we easily see, since $\Phi^{(-1)}=0$, that

$$
\left\{\begin{array}{c}
\Delta_{j,-}^{(0)}=\partial_{s} u_{j}^{(0)}\left(-\frac{L}{2}\right)+\frac{\mu_{j}}{2} \partial_{s} u_{j-\frac{1}{2}}^{(0)}+\sum_{\delta \in\{+,-, 0\}}\left\langle g_{j, \delta}^{(0)}, \mathcal{N}_{j}^{-}\right\rangle_{\Sigma_{j, \delta}}, \\
\Delta_{j,+}^{(0)}=\frac{\mu_{j}}{2} \partial_{s} u_{j+\frac{1}{2}}^{(0)}-\partial_{s} u_{j}^{(0)}\left(-\frac{L}{2}\right)+\sum_{\delta \in\{+,-, 0\}}\left\langle g_{j, \delta}^{(0)}, \mathcal{N}_{j}^{+}\right\rangle_{\Sigma_{j, \delta}}
\end{array}\right.
$$

Therefore, as a consequence of (93) and (98)-(99), we can write

$$
\Delta_{j, \pm}^{(0)}=r^{j} \hat{\Delta}_{ \pm}^{(0)} \quad j \in \mathbb{N}
$$

where $\hat{\Delta}_{ \pm}^{(0)}$ can be explicitly determined as a function of $\left(a_{0}^{(0)}, b_{0}^{(0)}, c_{0}^{(0)}\right)$ and $\lambda^{(0)}$. Moreover, using the symmetry properties of $\mathcal{N}_{j}^{ \pm}$(see Remark 6), we can see that for $j \in \mathbb{Z}$,

$$
\Delta_{-j,-}^{(0)}=-\Delta_{j,+}^{(0)} \quad \text { and } \quad \Delta_{-j,+}^{(0)}=-\Delta_{j,-}^{(0)}
$$

In particular we deduce that $\left\{\Delta_{j, \pm}^{(0)}\right\}_{j \in \mathbb{Z}} \in l_{2}(\mathbb{Z})$. Finally, we prove below that

$$
\forall j \in \mathbb{Z}, \quad \Xi_{j}^{(0)}=0
$$

Indeed, since $\Phi_{j}^{(0)}=-\lambda^{(0)} U_{j}^{(0)}$ (see (65)) and $U_{j}^{(0)} \equiv r^{|j|}$ (the constant function), using definition (77)(i), we obtain (we use also meas $K_{j}=\mu_{j}$ )

$$
\Xi_{j}^{(0)}=-\left\langle g_{j,+, \text { part }}^{(0)}, 1\right\rangle_{\Sigma_{j,+}}-\left\langle g_{j,-, \text { part }}^{(0)}, 1\right\rangle_{\Sigma_{j,-}}-\left\langle g_{j, 0, \text { part }}^{(0)}, 1\right\rangle_{\Sigma_{j, 0}}-\mu_{j} \lambda^{(0)} r^{|j|} .
$$

Using the definition (68) for $g_{j, 0, \text { part }}^{(0)}$ and the definition of $T_{j}^{0}$, we have

$$
\left\langle g_{j, 0, \text { part }}^{(0)}, 1\right\rangle_{\Sigma_{j, 0}}=\left\langle\left(\partial_{n}+T_{j}^{0}\right) U_{j, 0, \text { part }}^{(0)}, 1\right\rangle_{\Sigma_{j, 0}} \equiv\left\langle\partial_{n} U_{j, 0, \text { part }}^{(0)}, 1\right\rangle_{\Sigma_{j, 0}}
$$

Next, according to Proposition 2, $U_{j, 0, \text { part }}^{(0)}$ is obtained from the decomposition (44) of $U_{j}^{(2)}$ for $k=2$ and is solution of (46)-(47)-(48). It is then easy to see that

$$
U_{j, 0, \text { part }}^{(0)}(\mathfrak{s}, \mathfrak{t})=-\lambda^{(0)} r^{|j|} \frac{\mathfrak{s}^{2}}{2}+\sum_{\ell=1}^{+\infty} \hat{U}_{j, \ell, 0, \text { part }}^{(0)}(\mathfrak{s}) e^{-\frac{\ell \pi}{\mu_{j}}|\mathfrak{s}|} \mathbf{v}_{\ell, 0}^{j}(\mathfrak{t})
$$

Then, since $\Sigma_{j, 0}$ corresponds to $\mathfrak{s}=1$, we find that

$$
\left\langle g_{j, 0, \text { part }}^{(0)}, 1\right\rangle_{\Sigma_{j, 0}}=-\lambda^{(0)} r^{|j|} \mu_{j}
$$


Proceeding in the same way in the bands $B_{j, \pm}$ where $\partial_{n}= \pm \partial_{\mathfrak{s}}$, we find that

$$
\left\langle g_{j,+, \text { part }}^{(0)}, 1\right\rangle_{\Sigma_{j,+}}=-\frac{\mu_{j}}{2} \lambda^{(0)} r^{|j|}, \quad\left\langle g_{j,-, \text { part }}^{(0)}, 1\right\rangle_{\Sigma_{j,-}}=\frac{\mu_{j}}{2} \lambda^{(0)} r^{|j|}
$$

The relations (104) and (105) enables to conclude that $\Xi_{j}^{(0)}=0$ for all $j \in \mathbb{Z}$.

Thanks to (97), (100) and (102), the assumptions of Proposition 5 are satisfied for $k=1$ so that we can claim that there exists a unique $\left(\lambda^{(1)}, u^{(1)}\right)$, up to a normalization condition for $u^{(1)}$ (cf. Remark 7) that we will specify below (see (115)). Moreover, according to (88), $\lambda^{(1)}$ is given by

$$
\lambda^{(1)}=2\left\|u^{(0)}\right\|_{L_{2}^{\mu}\left(\mathcal{G}^{-}\right)}^{-2}\left(\sum_{j \in \mathbb{Z}} \widetilde{\Xi}_{j}^{(0)} \mathbf{u}_{j}^{(0)}\right),
$$

where, according to (89) and (100), we compute that

$$
\forall j \in \mathbb{N}, \quad \widetilde{\Xi}_{j}^{(0)}=-r^{j} \frac{\sqrt{\lambda^{(0)}}}{\sin \sqrt{\lambda^{(0)}}}\left(r \hat{\Delta}_{-}^{(0)}-r^{-1} \hat{\Delta}_{+}^{(0)}+\cos \sqrt{\lambda^{(0)}}\left(\hat{\Delta}_{+}^{(0)}-\hat{\Delta}_{-}^{(0)}\right)\right) .
$$

We can also give an explicit formula for $u^{(1)}$. First, from properties (101), we can claim that $u^{(1)}$ satisfy the symmetry property (91), so that we can restrict ourselves in the following to $j \geqslant 0$.

Let us first consider the two linear ordinary differential equations of (85) for $k=1$, namely:

$$
\left\{\begin{array}{ll}
\partial_{s}^{2} u_{j+\frac{1}{2}}^{(1)}(s)+\lambda^{(0)} u_{j+\frac{1}{2}}^{(1)}(s)=-\lambda^{(1)} u_{j+\frac{1}{2}}^{(0)}(s), & s \in(0,1), \\
\partial_{y}^{2} u_{j}^{(1)}(y)+\lambda^{(0)} u_{j}^{(1)}(y)=-\lambda^{(1)} u_{j}^{(0)}(y), & y \in(-L / 2,0),
\end{array} \quad j \in \mathbb{N} .\right.
$$

Using (93), we first compute a particular solution of (107), namely

$$
\left\{\begin{array}{l}
u_{j+\frac{1}{2}, \text { part }}^{(1)}(s)=\lambda^{(1)} r^{j} \frac{1}{2 \sqrt{\lambda^{(0)}}}\left(-a_{0}^{(0)} s \sin \left(\sqrt{\lambda^{(0)}} s\right)+b_{0}^{(0)} s \cos \left(\sqrt{\lambda^{(0)}} s\right)\right), \\
u_{j, \text { part }}^{(1)}(y)=\lambda^{(1)} r^{j} \frac{1}{2 \sqrt{\lambda^{(0)}}}\left(-c_{0}^{(0)} y \sin \left(\sqrt{\lambda^{(0)}} y\right)\right) .
\end{array}\right.
$$

Therefore, there exist $\left.\left.\left.\left\{\check{a}_{0}^{(1)}(j), \check{b}_{0}^{(1)}(j), j \in \mathbb{N}\right\}\right),\left\{\check{c}_{0}^{(1)}(j), \check{d}_{0}^{(1)}(j)\right), j>0\right\}\right)$ and $\left\{\check{c}_{0,0}^{(1)}, \check{d}_{0,0}^{(1)}\right\}$ such that

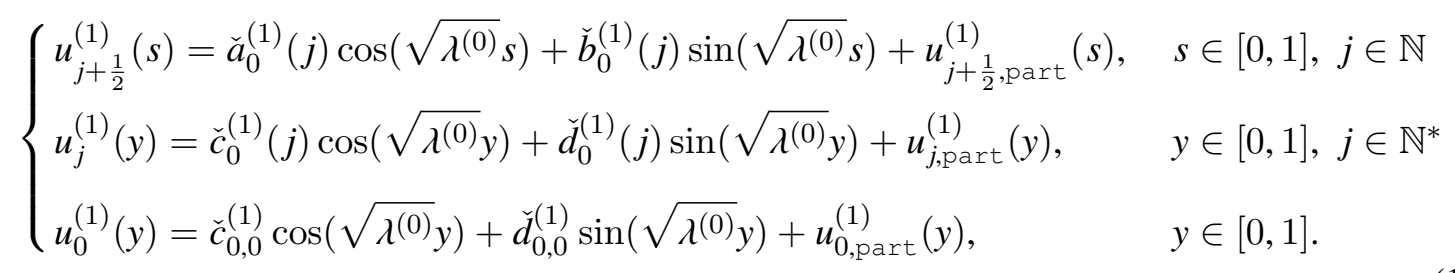


Let us now determine the above coefficients. First, (85)-(iii) (Neumann b. c.) leads to

$$
\check{d}_{0}^{(1)}(j)=0, \quad \forall j \in \mathbb{Z}, \quad \check{d}_{0,0}^{(1)}=0 .
$$

Next, (85)-(v) (the jump conditions for $j \in \mathbb{N}^{*}$ ) give, after some manipulations,

$$
\left\{\begin{array}{l}
\check{c}_{0}^{(1)}(j)=\frac{1}{\cos \left(\sqrt{\lambda^{(0)}} L / 2\right)} \check{a}_{0}^{(1)}(j)+c_{1} r^{j}, \quad \forall j \in \mathbb{N}^{*} \\
\check{b}_{0}^{(1)}(j)=\check{a}_{0}^{(1)}(j+1) \frac{1}{\sin \left(\sqrt{\lambda^{(0)}}\right)}-\check{a}_{0}^{(1)}(j) \frac{1}{\tan \left(\sqrt{\lambda^{(0)}}\right)}+b_{1} r^{j}, \quad \forall j \in \mathbb{N},(i i)
\end{array}\right.
$$

where the constants $b_{1}$ and $c_{1}$ are explicit functions of $\lambda^{(0)}$ (whose expression is omitted on purpose). To be more precise, (110)-( $i$ ) directly follows from the first jump condition while (110)-(ii) is deduced from the second jump condition at $j+1$ taken into account (110)-(i).

Finally, substituting (110) in the fourth equation in (85) (the Kirchhoff condition) yields to

$$
\check{a}_{0}^{(1)}(j+1)+2 g\left(\sqrt{\lambda^{(0)}}\right) \check{a}_{0}^{(1)}(j)+\check{a}_{0}^{(1)}(j-1)=r^{j} a_{1}, \quad \forall j \in \mathbb{N}^{*}
$$

where, again, $a_{1}$ is an explicit function of $\lambda^{(0)}$ and $g$ is defined in (13). Using that $r^{2}+2 g\left(\sqrt{\lambda^{(0)}}\right) r+1=$ 0 , a direct computation shows that a particular solution of the difference equation (111) is given by

$$
\check{a}_{0, p a r t}(j)=\frac{r a_{1}}{r^{2}-1} j r^{j}, \quad \forall j \in \mathbb{N} .
$$

As a result $a(j):=\check{a}_{0}^{(1)}(j)-\check{a}_{0, p a r t}(j)$ satisfies

$$
a(j+1)+2 g\left(\sqrt{\lambda^{(0)}}\right) a(j)+a(j-1)=0 \quad \forall j \in \mathbb{N}^{*}
$$

whose general solution in $\ell_{2}(\mathbb{N})$ has the form $\alpha_{0}^{(1)} r^{j}$. In other words, we have then proved that

$$
\check{a}_{0}^{(1)}(j)=r^{j} a_{0}^{(1)}(j), \quad \text { with } a_{0}^{(1)}(j):=\alpha_{0}^{(1)}+\frac{r a_{3}}{r^{2}-1} j, \quad \forall j \in \mathbb{N},
$$

Substituting (113) into (110) and (109), we obtain the formulas of the first two lines of (95) where we observe that

$$
a_{\ell}^{(1)}(j), b_{\ell}^{(1)}(j), c_{\ell}^{(1)}(j) \text { and } d_{\ell}^{(1)}(j) \text { are polynomials of degree } \leqslant 1-\ell \text { with respect to } j .
$$

Except the constant coefficient of $a_{0}^{(1)}(j)$ (coefficient of the polynomial $a_{0}^{(1)}(j)$ associated with the monomial of degree 0 ), these polynomials are explicitly determined by formulas (110)-(113). It is consistent with the fact that Problem (85) has a solution up to a multiple of $u^{(0)}$. At this stage, we can choose

$$
a_{0}^{(1)}(0)=0,
$$


where by definition $a_{0}^{(1)}(0)=u_{1 / 2}^{(1)}(0)$ as our normalisation choice (see Remark 7 with $\ell: u \mapsto u_{1 / 2}(0)$ ). It remains to compute $\check{c}_{0,0}^{(1)}$ for which we use the jump conditions and the Kirchhoff condition for $j=0$. A priori, this gives 3 linear equations in $\check{c}_{0,0}^{(1)}$. However, these three equations reduce to a single one allowing us to compute this coefficient explicitely. The verification of this property can be done by hand using the symmetry properties together with the fact that $\lambda^{(1)}$ is given by (106). However, this property is a consequence of Proposition 5, that ensures that there exists a unique solution to (85) (for $k=1$ ) satisfying the normalisation condition (115).

\subsubsection{Determination of $U^{(1)}$}

We already know that $\left(u^{(0)}, \lambda^{(0)}\right)$ satisfy (31), (75) and (76). In the same way, as solutions of (85) for $k=1,\left(u^{(1)}, \lambda^{(1)}\right)$ also satisfy (31), (75) and (76).

Since $U^{(0)}$, obtauned by concatenation of the constant functions $U_{j}^{(0)}$ (see (94)) satisfies (36), (56), we have checked that $\left(H_{1}\right)$ is satisfied. In view of Proposition 4 , we can thus assert that,

$\forall j \in \mathbb{Z}, \quad \exists ! U_{j}^{(1)} \in \mathcal{V}_{j}$ solution of (69) for $k=1$ satisfying the matching conditions (54-55). (116)

Moreover, $U_{j}^{(1)}$ is shown to be symmetric in the sense of (92) by using the symmetry of $u^{(0)}$ and $u^{(1)}$. At this stage, we distinguish between the computation of $U_{0}^{(1)}$ and the computation of $U_{j}^{(1)}, j \neq 0$.

The computation of $U_{0}^{(1)}$ is obtained by solving (69), for $k=1, j=0$, providing the restriction of $U_{0}^{(1)}$ to the junction $K_{0}$. Then, it is extended to the whole domain $\mathcal{J}_{0}$ as in the proof of Proposition 4.

Let us now consider the case $j \neq 0$ and prove (96) by showing that there exists a profile function $\mathcal{U}^{(1)} \in \mathcal{V}_{j} \equiv \mathcal{V}_{1}$ (with $K_{j}$ identified with $K_{1}$ ) and a constant $\mathcal{P}^{(1)}(j)$, that is a polynomial of degree 1 with respect to $j$, such that the decomposition (96) holds.

We first establish this decomposition inside $K_{j}$ and then extend it to the domain $\mathcal{J}_{j}$ as in the proof of Proposition 4, Section 3.2. As for $U_{j}^{(0)}$, this (straightforward) second step will be omitted.

For $j \in \mathbb{N}^{*}$, according to Section 3.2 and (98), we know that $\check{U}_{j}^{(1)}$, the restriction of $U_{j}^{(1)}$ in $K_{j}$ satisfies

$$
\begin{cases}\Delta \check{U}_{j}^{(1)}=0 & \text { in } K_{j}, \\ \partial_{n} \check{U}_{j}^{(1)}+T_{j}^{\delta} \check{U}_{j}^{(1)}=r^{j} g_{ \pm}^{(\delta)} & \text { on } \Sigma_{j, \delta}, \quad \delta=0, \pm, \\ \partial_{n} U_{j}^{(1)}=0 & \text { on } \partial K_{j} \backslash\left(\Sigma_{j, \pm} \cup \Sigma_{j, 0}\right) .\end{cases}
$$

as well as the following condition, which is nothing but the matching conditions (see (81)-(iii))

$$
\int_{\Sigma_{j, 0}} U_{j}^{(1)}=u_{j}^{(1)}\left(-\frac{L}{2}\right)+\partial_{y} u_{j}^{(0)}\left(-\frac{L}{2}\right) .
$$


From (93)-(95), one easily finds explicitly a polynomial $\mathcal{P}^{(1)}$ of degree 1 such that

$$
\int_{\Sigma_{j, 0}} U_{j}^{(1)}=r^{j} \mathcal{P}^{(1)}(j)
$$

The identification of $K_{j}$ with $K_{1}$ for $j \neq 0$ suggests of course to introduce the profile function $\check{\mathcal{U}}^{(1)}$ as the unique solution in $H^{1}\left(K_{1}\right)$ of (note that the well-posedness of (117) ensures the one of (119)))

$$
\begin{cases}\Delta \check{\mathcal{U}}^{(1)}=0 & \text { in } K_{1}, \\ \partial_{n} \check{\mathcal{U}}^{(1)}+T_{j}^{\delta} \check{\mathcal{U}}^{(1)}=g_{ \pm}^{(\delta)} & \text { on } \Sigma_{j, \delta}, \quad \delta=0,+,-\quad \text { (cf. (99)) } \\ \partial_{n} \check{\mathcal{U}}^{(1)}=0 & \text { on } \partial K_{j} \backslash\left(\Sigma_{j, \pm} \cup \Sigma_{j, 0}\right),\end{cases}
$$

completed with $\int_{\Sigma_{1,0}} \mathcal{U}^{(1)}=0$, So, using (118), by linearity $\check{U}_{j}^{(1)}=r^{j} \check{\mathcal{U}}^{(1)}+r^{j} \mathcal{P}^{(1)}(j)$.

\subsection{Order $k$ : the general induction step}

The previous reasoning can be repeated for any $k \geqslant 2$. As for $k=1$, we shall construct in turn

(1) the coefficient $\lambda^{(k)}$ and the far field terms $u^{(k)}$ by means of an explicit resolution of the far field problem (85) (see Section 3.3). In particular, we prove that $u^{(k)}$ is symmetric and

$$
\begin{cases}u_{j+\frac{1}{2}}^{(k)}(s)=r^{j} \sum_{\ell=0}^{k} s^{\ell}\left(a_{\ell}^{(k)}(j) \cos \left(\sqrt{\lambda_{0}} s\right)+b_{\ell}^{(k)}(j) \sin \left(\sqrt{\lambda_{0}} s\right)\right), & s \in[0,1], \quad j \in \mathbb{N}, \\ u_{j}^{(k)}(y)=r^{j} \sum_{\ell=0}^{k} y^{\ell}\left(c_{\ell}^{(k)}(j) \cos \left(\sqrt{\lambda_{0}} y\right)+d_{\ell}^{(k)}(j) \sin \left(\sqrt{\lambda_{0}} y\right)\right), & y \in\left[-\frac{L}{2}, 0\right], j \in \mathbb{N}^{*}, \\ u_{0}^{(k)}(y)=\sum_{\ell=0}^{k} y^{\ell}\left(c_{\ell, 0}^{(k)} \cos \left(\sqrt{\lambda_{0}} y\right)+d_{\ell, 0}^{(k)} \sin \left(\sqrt{\lambda_{0}} y\right)\right), & y \in\left[-\frac{L}{2}, 0\right] .\end{cases}
$$

the dependence with respect to the parameter $j$ being fully exhibited.

(2) the near field term $U^{(k)}$ (Section 4.3.3). We show that $U^{(k)}$ is symmetric (cf. (92)) and that

$$
U_{j}^{(k)}(\cdot)=r^{j}\left(\sum_{\ell=0}^{k-1} j^{\ell} \mathcal{U}_{\ell}^{(k)}(\cdot)+\mathcal{P}^{(k)}(j)\right) \quad j \in \mathbb{N}^{*}
$$

where $\mathcal{U}_{\ell}^{(k)} \in \mathcal{V}_{j} \equiv \mathcal{V}_{1}$ (see (35)) are profile functions independent of $j$ and $\mathcal{P}^{(k)}(j)$ is a polynomial of degree $k$ with respect to $j$ and constant with respect to $(X, Y) \in \mathcal{J}_{j}$.

We emphasize that the construction, although more technical, is similar to the one for $k=1$.

\subsubsection{Induction Assumptions}

First, we assume that the numbers $\lambda^{(n)}$, the far field terms $u^{(n)}$ and the near field terms $U^{(n)}$ are known up to order $n=k-1$, satisfy (31), (75), (76), (36) and (56) (with $n$ instead of $k$ ) and are symmetric in the sense of (91) and (92) respectively. 
We also assume that for all $n \leqslant k-1$, there exist polynomials $\left(a_{\ell}^{(n)}(\cdot), b_{\ell}^{(n)}(\cdot), c_{\ell}^{(n)}(\cdot), d_{\ell}^{(n)}(\cdot)\right)$ of degree $n-\ell, 0 \leqslant \ell \leqslant n$, and constants $\left(c_{\ell, 0}^{(n)}, d_{\ell, 0}^{(n)}\right), 0 \leqslant \ell \leqslant n$ such that

$$
\begin{cases}u_{j+\frac{1}{2}}^{(n)}(s)=r^{j} \sum_{\ell=0}^{n} s^{\ell}\left(a_{\ell}^{(n)}(j) \cos \left(\sqrt{\lambda_{0}} s\right)+b_{\ell}^{(k)}(j) \sin \left(\sqrt{\lambda_{0}} s\right)\right), & s \in[0,1], \quad j \in \mathbb{N}, \\ u_{j}^{(n)}(y)=r^{j} \sum_{\ell=0}^{n} y^{\ell}\left(c_{\ell}^{(n)}(j) \cos \left(\sqrt{\lambda_{0}} y\right)+d_{\ell}^{(n)}(j) \sin \left(\sqrt{\lambda_{0}} y\right)\right), & y \in\left[-\frac{L}{2}, 0\right], \quad j \in \mathbb{N}^{*} \\ u_{0}^{(n)}(y)=\sum_{\ell=0}^{n} y^{\ell}\left(c_{\ell, 0}^{(n)} \cos \left(\sqrt{\lambda_{0}} y\right)+d_{\ell, 0}^{(n)} \sin \left(\sqrt{\lambda_{0}} y\right)\right), & y \in\left[-\frac{L}{2}, 0\right] .\end{cases}
$$

Finally, we assume that there exists profile functions $\mathcal{U}_{\ell}^{(n)} \in \mathcal{V}_{j} \equiv \mathcal{V}_{1}$, for $0 \leqslant n \leqslant k-1$ and $0 \leqslant \ell \leqslant n-1$ and constants $\mathcal{P}^{(n)}(j), 0 \leqslant n \leqslant k-1$, that are polynomials of degree less than $n$ in $j$, such that

$$
U_{j}^{(n)}(\cdot)=r^{j}\left(\sum_{\ell=0}^{n-1} j^{\ell} \mathcal{U}_{\ell}^{(n)}(\cdot)+\mathcal{P}^{(n)}(j)\right) \quad j \in \mathbb{N}^{*} .
$$

\subsubsection{Determination of $\lambda^{(k)}$ and $u^{(k)}$}

They are obtained by solving (85). Let us investigate the structure of the data of this problem. First, using (68), one can verify that, for any $j \in \mathbb{Z}$,

$$
g_{-j, 0}^{(k-1)}=g_{j, 0}^{(k-1)} \quad g_{j,+}^{(k-1)}=g_{-j,-}^{(k-1)}, \quad \text { and } \quad g_{j, 0, \text { part }}^{(k-1)}=g_{-j, 0, \text { part }}^{(k-1)} \quad g_{j,+, \text { part }}^{(k-1)}=g_{-j,-, \text { part }}^{(k-1)},
$$

and, thanks to (122), for any $\delta \in\{0,+,-\}$, there exists a family of $k$ functions (explicit) $\hat{g}_{\delta, \ell}^{(k-1)}$, independent of $j$, such that

$$
g_{j, \delta}^{(k-1)}=r^{j} \sum_{\ell=0}^{k-1} j^{\ell} \hat{g}_{\delta, \ell}^{(k-1)} \quad \forall j \in \mathbb{N}^{*} .
$$

In the same way, the fields $\Phi_{j}^{(k-2)}$ have the symmetry property (92) and of the form

$$
\Phi_{j}^{(k-2)}=r^{j} \sum_{\ell=0}^{k-2} j^{\ell} \hat{\Phi}_{\ell}^{(k-2)}, \quad \forall j \in \mathbb{N}^{*}, \quad \text { with } \quad \hat{\Phi}_{\ell}^{(k-2)}=-\sum_{m=0}^{k-2} \lambda^{(k-m-2)} \mathcal{U}_{j}^{(m)}
$$

where the profile functions functions $\mathcal{U}_{j}^{(m)}$ are the ones appearing in (123). Then, using (77), we deduce from (125) and (126) that, for any $j \in \mathbb{Z}$,

$$
\Xi_{j}^{(k-1)}=\Xi_{-j}^{(k-1)}, \quad \Delta_{j,+}^{(k-1)}=-\Delta_{j,-}^{(k-1)},
$$

and there exist polynomials $\hat{\Xi}^{(k-1)}(\cdot)$ and $\hat{\Delta}_{ \pm}^{(k-1)}(\cdot)$ of degree at most $k-1$ such that

$$
\Xi_{j}^{(k-1)}=r^{j} \hat{\Xi}^{(k-1)}(j), \quad \Delta_{ \pm}^{(k-1)}=r^{j} \hat{\Delta}_{ \pm}^{(k-1)}(j), \quad \forall j \in \mathbb{N}^{*}
$$


Finally, it is easily seen that $f^{(k-1)}$ satisfies the symmetry property (90)(i) and that

$$
\begin{cases}f_{j+\frac{1}{2}}^{(k-1)}(s)=r^{j} \sum_{\ell=0}^{k-1} s^{\ell}\left(\hat{\mathfrak{a}}_{\ell}^{(k-1)}(j) \cos \left(\sqrt{\lambda_{0}} s\right)+\hat{\mathfrak{b}}_{\ell}^{(k-1)}(j) \sin \left(\sqrt{\lambda_{0}} s\right)\right), & s \in[0,1], \quad j \in \mathbb{N}, \\ f_{j}^{(k-1)}(y)=r^{j} \sum_{\ell=0}^{k-1} y^{\ell}\left(\hat{\mathfrak{c}}_{\ell}^{(n)}(j) \cos \left(\sqrt{\lambda_{0}} y\right)+\hat{\mathfrak{d}}_{\ell}^{(k-1)}(j) \sin \left(\sqrt{\lambda_{0}} y\right)\right), & y \in\left[-\frac{L}{2}, 0\right], j \neq 0, \\ f_{0}^{(k-1)}(y)=\sum_{\ell=0}^{k-1} y^{\ell}\left(\hat{\mathfrak{c}}_{\ell, 0}^{(k-1)} \cos \left(\sqrt{\lambda_{0}} y\right)+\hat{\mathfrak{d}}_{\ell, 0}^{(k-1)} \sin \left(\sqrt{\lambda_{0}} y\right)\right), & y \in\left[-\frac{L}{2}, 0\right]\end{cases}
$$

where the coefficients $\hat{\mathfrak{a}}_{\ell}^{(k-1)}(j), \hat{\mathfrak{b}}_{\ell}^{(k-1)}(j), \hat{\mathfrak{c}}_{\ell}^{(k-1)}(j), \hat{\mathfrak{d}}_{\ell}^{(k-1)}(j), \hat{\mathfrak{c}}_{\ell, 0}^{(k-1)}$ and $\hat{\mathfrak{d}}_{\ell, 0}^{(k-1)}$ are explicitly computed in function of the coefficients appearing in (122) for $n \leqslant k-1$ Formulas (128) and (129) ensure that

$$
\left\{\Xi_{j}^{(k-1)}\right\}_{j \in \mathbb{Z}} \in l_{2}(\mathbb{Z}), \quad\left\{\Delta_{j, \pm}^{(k-1)}\right\}_{j \in \mathbb{Z}} \in l_{2}(\mathbb{Z}) \text { and } f^{(k-1)} \in L_{2}^{\mu}\left(\mathcal{G}^{-}\right) .
$$

Thus the assumptions of Proposition 5 are satisfied, so that there exists a unique $\left(\lambda^{(k)}, u^{(k)}\right)$, up to a normalization condition for $u^{(k)}$ (see Remark 7) that we will specify below (see (139)). Moreover, $\lambda^{(k)}$ is given by (88). Because of the symmetry properties (127), Lemma 5 ensures that $u^{(k)}$ is symmetric.

Next, we prove (120). The first two equations of (85) are non homogenenous linear second order ordinary differential equations that we are able to solve explicitly.

First, we determine the unique particular solutions $u_{j+\frac{1}{2} \text {,part }}^{(k)}$ and $u_{j, \mathrm{part}}^{(k)}$ of (85) that have the form

$$
\begin{cases}u_{j+\frac{1}{2}, \text { part }}^{(k)}(s)=r^{j} \sum_{\ell=1}^{k} s^{\ell}\left(a_{\ell}^{(k)}(j) \cos \left(\sqrt{\lambda_{0}} s\right)+b_{\ell}^{(k)}(j) \sin \left(\sqrt{\lambda_{0}} s\right)\right), s \in[0,1], \quad j \in \mathbb{N}, \\ u_{j, \text { part }}^{(k)}(y)=r^{j} \sum_{\ell=1}^{k} y^{\ell}\left(c_{\ell}^{(k)}(j) \cos \left(\sqrt{\lambda_{0}} y\right)+d_{\ell}^{(k)}(j) \sin \left(\sqrt{\lambda_{0}} y\right)\right), & y \in\left[-\frac{L}{2}, 0\right], j \neq 0, \\ u_{0, \text { part }}^{(k)}(y)=\sum_{\ell=1}^{k} y^{\ell}\left(c_{\ell, 0}^{(k)} \cos \left(\sqrt{\lambda_{0}} y\right)+d_{\ell, 0}^{(k)} \sin \left(\sqrt{\lambda_{0}} y\right)\right), & y \in\left[-\frac{L}{2}, 0\right],\end{cases}
$$

where the constants $a_{\ell}^{(k)}(j), b_{\ell}^{(k)}(j), c_{\ell}^{(k)}(j)$ and $d_{\ell}^{(k)}(j)$, with $1 \leqslant \ell \leqslant k$ are polynomials in $j$ of degree at most $k-\ell$. The computation of the coefficients in (130) is straightforward but quite tedious. It will be omitted here but can be found in [27]. Next, we know that there are new coefficients (indexed by 0 and indicated with a tilde) such that

$$
\left\{\begin{array}{lll}
u_{j+\frac{1}{2}}^{(k)}(s)=\check{a}_{0}^{(k)}(j) \cos \left(\sqrt{\lambda^{(0)}} s\right)+\check{b}_{0}^{(k)}(j) \sin \left(\sqrt{\lambda^{(0)}} s\right)+u_{j+\frac{1}{2}, \text { part }}^{(k)}(s), & s \in[0,1], & j \in \mathbb{N} \\
u_{j}^{(k)}(y)=\check{c}_{0}^{(k)}(j) \cos \left(\sqrt{\lambda^{(0)}} y\right)+\check{d}_{0}^{(k)}(j) \sin \left(\sqrt{\lambda^{(0)}} y\right)+u_{j, \mathrm{part}}^{(k)}(y), & y \in[0,1], & j \in \mathbb{N}^{*} \\
u_{0}^{(k)}(y)=\check{c}_{0,0}^{(k)} \cos \left(\sqrt{\lambda^{(0)}} y\right)+\check{d}_{0,0}^{(k)} \sin \left(\sqrt{\lambda^{(0)}} y\right)+u_{0, \mathrm{part}}^{(k)}(y), & y \in[0,1] . &
\end{array}\right.
$$

Now, we determine these coefficients. First, (85)-(iii) (the Neumann boundary condition) leads to

$$
\check{d}_{0}^{(k)}(j)=-\left(u_{j, \text { part }}^{(k)}\right)^{\prime}(0)=r^{j} d_{0}^{(k)}(j), \quad \forall j \in \mathbb{N}^{*}, \quad \text { and } \quad \check{d}_{0,0}^{(k)}=-\left(u_{0, \text { part }}^{(k)}\right)^{\prime}(0),
$$


where $d_{0}^{(k)}(j)$ is a polynomial of degree at most $k-1$, computed from the $a_{\ell}^{(k)}(\cdot), b_{\ell}^{(k)}(\cdot), c_{\ell}^{(k)}(\cdot), d_{\ell}^{(k)}(\cdot) \quad 1$ for $1 \leqslant \ell \leqslant k$. Next, after the same manipulations as in the case $k=1$, equations (85)-(v) (the jump conditions for $j \in \mathbb{N}^{*}$ ) give

$$
\left\{\begin{array}{l}
\check{c}_{0}^{(k)}(j)=\frac{1}{\cos \left(\sqrt{\lambda^{(0)}} L / 2\right)} \check{a}_{0}^{(k)}(j)+c_{k}(j) r^{j}, \quad \forall j \in \mathbb{N}^{*} \\
\check{b}_{0}^{(k)}(j)=\check{a}_{0}^{(k)}(j+1) \frac{1}{\sin \left(\sqrt{\lambda^{(0)}}\right)}-\check{a}_{0}^{(k)}(j) \frac{1}{\tan \left(\sqrt{\lambda^{(0)}}\right)}+b_{k}(j) r^{j}, \quad \forall j \in \mathbb{N},(i i)
\end{array}\right.
$$

where $c_{k}(j)$ and $b_{k}(j)$ are polynomials in $j$ of degree at most $k-1$ that, again, can be explicitly computed. The formulas can be found in [27]. Let us simply point out here that these polynomials are deduced from the particular solution (131), i. e.

$$
\left\{\begin{array}{l}
\text { The polynomials } c_{k}(\cdot) \text { and } b_{k}(\cdot) \text { are explicitly defined as explicit (and linear) } \\
\text { functions of the polynomials } a_{\ell}^{(k)}(\cdot), b_{\ell}^{(k)}(\cdot), c_{\ell}^{(k)}(\cdot), d_{\ell}^{(k)}(\cdot) \text { for } 1 \leqslant \ell \leqslant k .
\end{array}\right.
$$

Finally, substituting (133) in the fourth equation in (85) (the Kirchhoff condition) yields to

$$
\check{a}_{0}^{(k)}(j+1)+2 g\left(\sqrt{\lambda^{(0)}}\right) \check{a}_{0}^{(k)}(j)+\check{a}_{0}^{(k)}(j-1)=r^{j} a_{k}(j), \quad \forall j \in \mathbb{N}^{*},
$$

where $a_{k}(j)$ is a polynomial with respect to $j$ of degree at most $k-1$. Using that $r^{2}+2 g\left(\sqrt{\lambda^{(0)}}\right) r+1=0$, a direct computation shows that there exists a particular solution of (135) which is of the form

$$
\check{a}_{\text {part }}^{(k)}(j)=r^{j} \sum_{\ell=1}^{k} \alpha_{\ell}^{(k)} j^{\ell} \quad \forall j \in \mathbb{N}
$$

where the coefficients $\alpha_{\ell}^{(k)}$ can be computed by solving a square invertible linear system of size $k$ (details are left to the reader).

As a result $a(j):=\check{a}_{0}^{(k)}(j)-\check{a}_{\text {part }}^{(k)}(j)$ satisfies

$$
a(j+1)+2 g\left(\sqrt{\lambda^{(0)}}\right) a(j)+a(j-1)=0 \quad \forall j \in \mathbb{N}^{*}
$$

whose general solution in $\ell_{2}(\mathbb{N})$ has the form $\alpha_{0}(k) r^{j}$ for any $j \in \mathbb{N}$. Thus, we have then proved that

$$
\check{a}_{0}^{(k)}(j)=r^{j} a_{0}^{(k)}(j) \quad \text { with } a_{0}^{(k)}(j):=\alpha_{0}^{(k)}+\sum_{\ell=1}^{k} \alpha_{\ell}^{(k)} j^{\ell}, \quad \forall j \in \mathbb{N}, \quad \forall j \in \mathbb{N} .
$$

Collecting (132)-(133)-(137) proves the first two lines of (120) with, according to (133)

$$
\left\{\begin{array}{l}
c_{0}^{(k)}(j)=\frac{1}{\cos \left(\sqrt{\lambda^{(0)}} L / 2\right)} a_{0}^{(k)}(j)+c_{k}(j), \quad \forall j \in \mathbb{N}^{*} \\
b_{0}^{(k)}(j)=r a_{0}^{(k)}(j+1) \frac{r}{\sin \left(\sqrt{\lambda^{(0)}}\right)}-a_{0}^{(k)}(j) \frac{1}{\tan \left(\sqrt{\lambda^{(0)}}\right)}+b_{k}(j) r^{j}, \quad \forall j \in \mathbb{N} .(i i)
\end{array}\right.
$$


Except the constant coefficient of $a_{0}^{(k)}(j)$ (seen as a polynomial in $j$ ), these polynomials are explicitly determined by formulas (132)-(133)-(137) . Here again, we choose

$$
a_{0}^{(k)}(0)=0
$$

where $a_{0}^{(k)}(0)=u_{1 / 2}^{(k)}(0)$ as our normalisation choice (see Remark 7 with $\ell: u \mapsto u_{1 / 2}(0)$ ).

To complete the determination of $u^{(k)}$, it only remains to compute $\check{c}_{0,0}^{(k)}$, (and thus $c_{0,0}^{(k)} \equiv \check{c}_{0,0}^{(k)}$ as it follows from comparing (120) with (130)-(131)). As for $k=1$, it is entirely determined by the Kirchhoff condition (85)-(iv) for $j=0$.

\subsubsection{Determination of $U^{(k)}$}

As solutions of (85), $\left(u^{(k)}, \lambda^{(k)}\right)$ also satisfy (31), (75) and (76). Joined to the recurrence assumptions, this shows that the assumption $\left(H_{k}\right)$ is satisfied and Proposition (4) allows us to say that

$\forall j \in \mathbb{Z}, \quad \exists ! U_{j}^{(k)} \in \mathcal{V}_{j}$ solution of (36) satisfying the matching conditions (58)-(60).

It is easy to see that, using the symmetry properties (124)-(127), $U_{j}^{(k)}$ is symmetric in the sense of (92).

As for $k=1$, the computation of $U_{0}^{(k)}$ is done independently proceeding as in Section 4.2.

Next we show (121). As in Section 4.2, we first establish such a decomposition inside $K_{j}$ before extending it to the domain $\mathcal{J}_{j}$ as in the proof of Proposition 4, Section 3.2.

For $j \in \mathbb{N}^{*}$, according to Section 3.2 and $(125), \check{U}_{j}^{(k)}$, the restriction of $U_{j}^{(k)}$ in $K_{j}$ satisfies

$$
\begin{cases}\Delta \check{U}_{j}^{(k)}=0 & \text { in } K_{j}, \\ \partial_{n} \check{U}_{j}^{(k)}+T_{j}^{\delta} \check{U}_{j}^{(k)}=r^{j} \sum_{\ell=0}^{k-1} j^{\ell} \hat{g}_{\delta, \ell}^{(k-1)} & \text { on } \Sigma_{j, \delta}, \quad \delta=0, \pm, \\ \partial_{n} U_{j}^{(k)}=0 & \text { on } \partial K_{j} \backslash\left(\Sigma_{j, \pm} \cup \Sigma_{j, 0}\right) .\end{cases}
$$

as well as the following condition (81)-(iii). Using Formulas (93)-(95), it is easily seen that there exists a polynomial $\mathcal{P}^{(k)}$ of degree $k$ that we can compute explicitly such that (81)-(iii) rewrites

$$
\int_{\Sigma_{j, 0}} \check{U}_{j}^{(k)}=r^{j} \mathcal{P}^{(k)}(j)
$$


Then, we introduce the $(k-1)$ profile functions $\check{\mathcal{U}}_{\ell}^{(k)}, 0 \leqslant \ell \leqslant k-1$ as the unique solutions (see Lemma 6 below) in $H^{1}\left(K_{1}\right)$ of the following problems

$$
\begin{cases}\Delta \check{\mathcal{U}}_{\ell}^{(k)}=0 & \text { in } K_{1}, \\ \partial_{n} \check{\mathcal{U}}_{\ell}^{(k)}+T_{j}^{\delta} \check{\mathcal{U}}^{(k)}=\hat{g}_{\delta, \ell}^{(k-1)} & \text { on } \Sigma_{j, \delta}, \quad \delta=0, \pm, \quad \text { (cf. (99)) (ii) } \\ \partial_{n} \check{\mathcal{U}}_{\ell}^{(k)}=0 & \text { on } \partial K_{j} \backslash\left(\Sigma_{j, \pm} \cup \Sigma_{j, 0}\right) . \\ \int_{\Sigma_{1,0}} \mathcal{U}_{\ell}^{(k)}=0 . & \text { (iii) }\end{cases}
$$

Then by linearity, it is straighforward to check that (121) holds in $K_{j}$, thus in the whole junction $\mathcal{J}_{j}$ too. To conclude, we need to come back to the well-posedness of (143), which is not as straightforward as the one of (119).

Lemma 6. Each of the problems (143), $0 \leqslant \ell \leqslant k-1$, admits a unique solution.

Proof. Instead of verifying the compatibility condition (71) of Lemma 3), we give an indirect proof that aims at exploiting the fact that the problems (117)- (118) for $j \in \mathbb{Z}$ are well posed.

For this, we shall exploit the $k$ fields $\check{U}_{j}^{(k)}, 1 \leqslant j \leqslant k$ and look for the solution $\check{\mathcal{U}}_{\ell}^{(k)}$ in the form

$$
\check{\mathcal{U}}_{\ell}^{(k)}=\sum_{j=1}^{k} \alpha_{\ell, j}^{(k)}\left(\check{U}_{j}^{(k)}-r^{j} \mathcal{P}^{(k)}(j)\right) .
$$

By construction $\check{\mathcal{U}}_{\ell}^{(k)}$ satisfies (143)-(i), (143)-(iii) and (143)-(iv). Only, (143)-(ii) needs to be checked. Substituting (144) into (143)-(ii), it is readily seen that this equation is satisfied as soon as

$$
\forall 0 \leqslant \ell \leqslant k-1, \quad \sum_{j=1}^{k} \alpha_{\ell, j}^{(k)} r^{j} \sum_{m=0}^{k-1} j^{m} \hat{g}_{\delta, m}^{(k-1)}=\hat{g}_{\delta, \ell}^{(k-1)}
$$

This defines uniquely the coefficients $\left(\alpha_{\ell, j}, 0 \leqslant \ell \leqslant k-1,1 \leqslant j \leqslant k\right)$ since the $k \times k$ Van der Monde matrix $A$ withentries $\left(r^{j} j^{m}\right), 1 \leqslant j \leqslant k, 0 \leqslant m \leqslant k-1$ is invertible (its determinant is, up to a strictly positive multiplicative constant, the product of $(j-i)$ for $1 \leqslant i<j \leqslant k$.

\section{Justification of the asymptotic expansion}

The existence of the (formal) asymptotic expansion being proved, we now prove Theorem 1 by first constructing (Section 5.1) pseudo-modes as defined in Section 2.1. Then we prove that (19) holds with (18). This is based on error estimates of Sections 5.2 and 5.3. This allows to conclude (Section 5.4).

\subsection{Construction of pseudomodes and related properties}

Roughly speaking, given $n>0$, we construct the approximate far and near fields "of order $n$ " by truncating the expansions (27), (28) and (32). More precisely, for the far field, we define : 


$$
\left\{\begin{array}{l}
u_{\mathrm{FF}}^{\varepsilon, n}: \Omega_{\varepsilon}^{\mu} \longrightarrow \mathbb{R}, \text { such that } u_{\mathrm{FF}}^{\varepsilon, n}(x, y)=0 \text { in } \mathcal{K}_{j}^{\varepsilon,-}, j \in \mathbb{Z} \text { and } \\
u_{\mathrm{FF}}^{\varepsilon, n}(x, y) \equiv u_{j+\frac{1}{2}}^{\varepsilon, n}(x, y):=\sum_{k=0}^{n} \varepsilon^{k} u_{j+\frac{1}{2}}^{(k)}(s), \quad s=x-j, \quad(x, y) \in \mathcal{E}_{j+\frac{1}{2}}^{-, \varepsilon}, \quad j \in \mathbb{Z}, \\
u_{\mathrm{FF}}^{\varepsilon, n}(x, y) \equiv u_{j}^{\varepsilon, n}(x, y):=\sum_{k=0}^{n} \varepsilon^{k} u_{j}^{(k)}(y), \quad, \quad(x, y) \in \mathcal{E}_{j}^{-, \varepsilon}, \quad j \in \mathbb{Z} .
\end{array}\right.
$$

In each "thick edge"of $\Omega_{\varepsilon}^{\mu}$, the couple $\left(u_{\mathrm{FF}}^{\varepsilon, n}, \lambda^{\varepsilon, n}\right)\left(\lambda^{\varepsilon, n}\right.$ defined in (19), see also below) does not exactly satisfy the desired eigenvalue equation because of the truncation process. More precisely, combining adequately the equations (31) for $0 \leqslant k \leqslant 2 n$, one finds that (the computations are tedious but straightforward, the details are left to the reader)

$$
\Delta u_{\mathrm{FF}}^{\varepsilon, n}+\lambda^{\varepsilon, n} u_{\mathrm{FF}}^{\varepsilon, n}=\varepsilon^{n+1} r_{\mathrm{FF}}^{\varepsilon, n}, \quad \text { in } \mathcal{O}_{\varepsilon}^{\mu}:=\bigcup_{j \in \mathbb{Z}}\left(\mathcal{E}_{j+\frac{1}{2}}^{-, \varepsilon} \cup \mathcal{E}_{j}^{-, \varepsilon}\right), \quad \lambda^{\varepsilon, n}:=\sum_{k=0}^{n} \varepsilon^{k} \lambda^{(k)},
$$

where the so-called "far-field remainder" $r_{\mathrm{FF}}^{\varepsilon, n}$ is such that $r_{\mathrm{FF}}^{\varepsilon, n}=0$ in $\mathcal{K}_{j}^{\varepsilon,-}$ and

$$
\left\{\begin{array}{l}
r_{\mathrm{FF}}^{\varepsilon, n}(x, y):=\sum_{p=n+1}^{2 n} \varepsilon^{p-n-1} r_{j+\frac{1}{2}}^{p, n}(x, y), \quad(x, y) \in \mathcal{E}_{j+\frac{1}{2}}^{-, \varepsilon}, \quad j \in \mathbb{Z}, \\
r_{\mathrm{FF}}^{\varepsilon, n}(x, y):=\sum_{p=n+1}^{2 n} \varepsilon^{p-n-1} r_{j}^{p, n}(x, y), \quad(x, y) \in \mathcal{E}_{j}^{-, \varepsilon}, \quad j \in \mathbb{Z}, \\
r_{j+\frac{1}{2}}^{p, n}(x, y)=\sum_{(k, \ell) \in \mathcal{I}^{p, n}} \lambda^{(k)} u_{j+\frac{1}{2}}^{(\ell)}(s) \quad s=x-j, \quad r_{j}^{p, n}(x, y)=\sum_{(k, \ell) \in \mathcal{I}^{p, n}} \lambda^{(k)} u_{j}^{(\ell)}(y)
\end{array}\right.
$$

where we have defined, with $\mathcal{I}^{p, n}=\left\{(\ell, k) \in\{0, \cdots, n\}^{2} / \ell+k=p\right\}$.

On the other hand, we define for $j \in \mathbb{Z}$ the truncated near fields $U_{j}^{\varepsilon, n}: \mathcal{J}_{j}^{\varepsilon}:=(j, 0)+\varepsilon \mathcal{J}_{j} \rightarrow \mathbb{R}$,

$$
U_{j}^{\varepsilon, n}(x, y)=\sum_{k=1}^{n} \varepsilon^{k} U_{j}^{(k)}\left(\frac{x-j}{\varepsilon}, \frac{y+L / 2}{\varepsilon}\right),
$$

Again, the couple $\left(U_{j}^{\varepsilon, n}, \lambda^{\varepsilon, n}\right)$ does not exactly satisfy the desired eigenvalue equation in $\mathcal{J}_{j}^{\varepsilon}$. Combining adequately the equations (36) for $0 \leqslant k \leqslant 2 n$, we get (see remark 10)

$$
\Delta U_{j}^{\varepsilon, n}+\lambda^{\varepsilon, n} U_{j}^{\varepsilon, n}=\varepsilon^{n-1} R_{j}^{\varepsilon, n}, \quad \text { in } \mathcal{J}_{j}^{\varepsilon}, \quad j \in \mathbb{Z} .
$$

where, reminding that $\mathcal{I}^{p, n}=\left\{(\ell, k) \in\{0, \cdots, n\}^{2} / \ell+k=p\right\}$, the "near-field remainder" $R_{j}^{\varepsilon, n}$ is

$$
\left\{\begin{array}{l}
R_{j}^{\varepsilon, n}(x, y):=\sum_{p=n-1}^{2 n} \varepsilon^{p-(n-1)} R_{j}^{p, n}\left(\frac{x-j}{\varepsilon}, \frac{y+L / 2}{\varepsilon}\right), \quad \text { in } \mathcal{J}_{j}^{\varepsilon}, \\
R_{j}^{p, n}:=\sum_{(k, \ell) \in \mathcal{I}^{p, n}} \lambda^{(k)} U_{j}^{(\ell)}, \quad \text { in } \mathcal{J}_{j} .
\end{array}\right.
$$


Remark 10. The computations that lead to (149) and (150) are of course quite similar to the ones that lead to (146) and (147). The reader will notice that the power of $\varepsilon$ that appears as the multiplying factor in the right hand sides of (146) and (149) passes from $n+1$ in (146) to $n-1$ (149). Accordingly, there is a difference in the numbers of terms in the sums that define the remainders which passes from $n-1$ in (147) to $n+1$ in(150). These changes are due, one on hand to the differences that already occur in equations (31) and (36) respectively, on the other hand to the E-scaling that appears in the definition (148) of the truncated near fields.

Next, we want to construct a pseudomode that will coincide with $u_{\mathrm{FF}}^{\varepsilon, n}$ outside some small neighborhood of the junctions $\mathcal{K}_{\varepsilon, j}^{-}$and with $U_{j}^{\varepsilon, n}$ in a neighborhood of $\mathcal{K}_{\varepsilon, j}^{-}$. This will be done in a smooth way with the help of cut-off functions and a partition of unity. Let us first introduce $\chi \in C^{\infty}(\mathbb{R})$ such that

$$
0 \leqslant \chi(x) \leqslant 1, \quad \forall x \in \mathbb{R}, \quad \chi(x)=0, x \leqslant 1, \quad \chi(x)=1, x \geqslant 2,
$$

from which we define the 2D cut-off functions ( $\alpha$ is a real parameter to be fixed later)

$$
\chi_{j}^{\varepsilon}(x, y)=\chi\left((x-j) / \varepsilon^{\alpha}\right) \chi\left((y+L / 2) / \varepsilon^{\alpha}\right), \quad j \in \mathbb{Z},
$$

where we impose $0<\alpha<1$ : since $\alpha<1$, the support of $\chi_{j}^{\varepsilon}$ is an $\varepsilon^{\alpha}$-neighborhood of $\mathcal{K}_{j}^{\varepsilon,-}$ and, since $\alpha>0$, these supports are disjoint for $\varepsilon$ small enough. This allows us to define

$$
\chi^{\varepsilon}=\sum_{j \in \mathbb{Z}} \chi_{j}^{\varepsilon}
$$

that coincides which $\chi_{j}^{\varepsilon}$ on its support. By construction $\left\{\left(1-\chi^{\varepsilon}\right),\left\{\chi_{j}^{\varepsilon}, j \in \mathbb{Z}\right\}\right\}$ form a partition of unity in $\mathbb{R}^{2}$. The properties of the cut-off functions are illustrated on Figures 7 and 8 . Multipying by $\left(1-\chi^{\varepsilon}\right)$ localizes outside the junctions, while multiplying by $\chi^{\varepsilon}$ (resp. $\chi_{j}^{\varepsilon}$ ) localizes near the junctions (resp. the $j^{\text {th }}$ - junction). In practice, the forthcoming analysis shows that it is advantageous to take $\alpha$ as close as possible to 1 (see estimates (155) and (170)-(ii)), the case $\alpha=1$ being excluded though (cf. (160))). This leads us to define the pseudo-mode of order $n$ as follows

$$
u^{\varepsilon, n}=\left(1-\chi^{\varepsilon}\right) u_{\mathrm{FF}}^{\varepsilon, n}+\sum_{j \in \mathbb{Z}} \chi_{j}^{\varepsilon} U_{j}^{\varepsilon, n}
$$

According to Section 2.1, our goal will be to get an estimate of the quantity

$$
\mathcal{I}^{\varepsilon, n}(v)=\int_{\Omega_{\varepsilon}^{\mu,-}}\left(\nabla u^{\varepsilon, n} \nabla v-\lambda^{\varepsilon, n} u^{\varepsilon, n} v\right) .
$$

To obtain a tractable expression $\mathcal{I}^{\varepsilon, n}(v)$, we first compute that

$$
\begin{aligned}
\Delta u^{\varepsilon, n}+\lambda^{\varepsilon, n} u^{\varepsilon, n} & =\left(1-\chi^{\varepsilon}\right)\left(\Delta u_{\mathrm{FF}}^{\varepsilon, n}+\lambda^{\varepsilon, n} u_{\mathrm{FF}}^{\varepsilon, n}\right)-2 \nabla \chi^{\varepsilon} \cdot \nabla u_{\mathrm{FF}}^{\varepsilon, n}-\Delta \chi^{\varepsilon} u_{\mathrm{FF}}^{\varepsilon, n} \\
& +\sum_{j \in \mathbb{Z}}\left[\chi_{j}^{\varepsilon}\left(\Delta U_{j}^{\varepsilon, n}+\lambda^{\varepsilon, n} U_{j}^{\varepsilon, n}\right)+2 \nabla \chi_{j}^{\varepsilon} \cdot \nabla U_{j}^{\varepsilon, n}+\Delta \chi_{j}^{\varepsilon} U_{j}^{\varepsilon, n}\right] .
\end{aligned}
$$



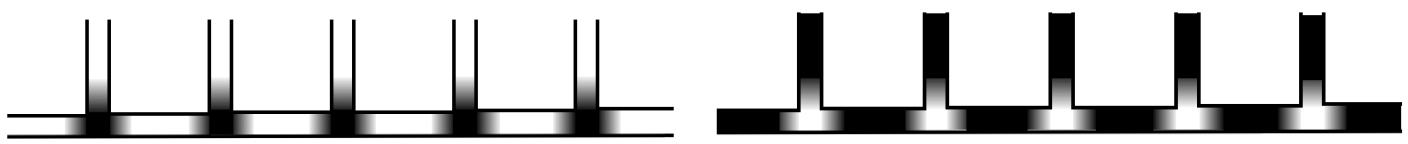

Fig. 7. Support of the function $\chi^{\varepsilon}$ (left) and $1-\chi^{\varepsilon}$ (right). White corresponds to 0 , black to 1
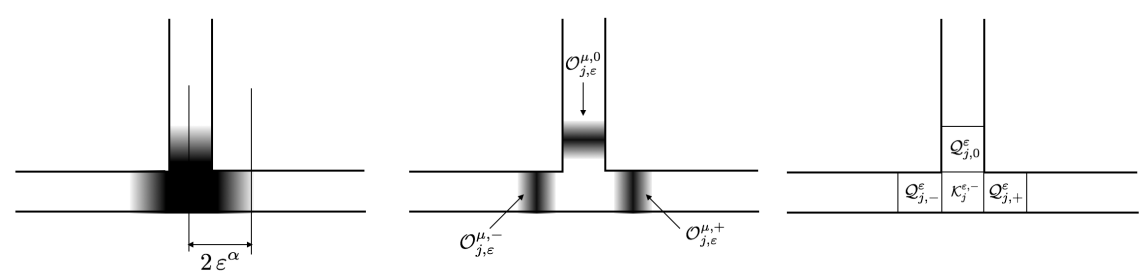

Fig. 8. Support of the function $\chi_{j, \varepsilon}$ (left) and of its gradient (right).

Using (146), (149) and the fact that, in $\operatorname{supp} \chi_{j}^{\varepsilon}, \chi^{\varepsilon} \equiv \chi_{j}^{\varepsilon}$, this can be rearranged as

$$
\begin{aligned}
\Delta u^{\varepsilon, n}+\lambda^{\varepsilon, n} u^{\varepsilon, n} & =\varepsilon^{n+1}\left(1-\chi^{\varepsilon}\right) r_{\mathrm{FF}}^{\varepsilon, n}+\varepsilon^{n-1} \sum_{j \in \mathbb{Z}} \chi_{j}^{\varepsilon} R_{j}^{\varepsilon, n} \\
& -2 \sum_{j \in \mathbb{Z}} \nabla \chi_{j}^{\varepsilon} \cdot \nabla\left(u_{\mathrm{FF}}^{\varepsilon, n}-U_{j}^{\varepsilon, n}\right)-\sum_{j \in \mathbb{Z}} \Delta \chi_{j}^{\varepsilon}\left(u_{\mathrm{FF}}^{\varepsilon, n}-U_{j}^{\varepsilon, n}\right) .
\end{aligned}
$$

Multiply the above equality by $-v \in H^{1}\left(\Omega_{\varepsilon}^{\mu}\right)$ and integrate over $\Omega_{\varepsilon}^{\mu}$. Using Green's formula, we get:

$$
\mathcal{I}^{\varepsilon, n}(v)=-\mathcal{I}_{\mathrm{FF}}^{\varepsilon, n}(v)-\mathcal{I}_{\mathrm{NF}}^{\varepsilon, n}(v)-\mathcal{I}_{\mathrm{M}}^{\varepsilon, n}(v),
$$

where by definition

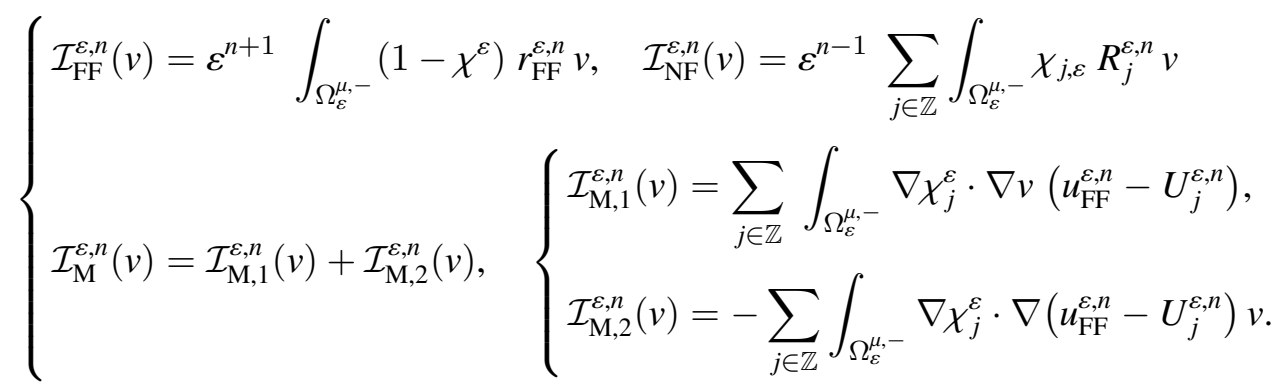

In formula (153), we say that :

$\mathcal{I}_{\mathrm{FF}}^{\varepsilon, n}(v)$ is the far field consistency error : it measures how much $\left(u^{\varepsilon, n}, \lambda^{\varepsilon, n}\right)$ fails to satisfy the desired eigenvalue equation inside the support of $1-\chi^{\varepsilon}$,

$\mathcal{I}_{\mathrm{NF}}^{\mathcal{E}, n}(v)$ is the near field consistency error : it measures how much $\left(u^{\varepsilon, n}, \lambda^{\varepsilon, n}\right)$ fails to satisfy the desired 
eigenvalue equation inside the support of $\chi^{\varepsilon}$,

$\mathcal{I}_{\mathrm{M}}^{\varepsilon, n}(v)$ is the matching error: it gathers the mismatch between $u_{\mathrm{FF}}^{\varepsilon, n}$ and $U_{j}^{\varepsilon, n}$ in supp $\chi_{j}^{\varepsilon}$ for all $j$.

\subsection{Estimation of the matching error.}

Lemma 7. The matching error $\mathcal{I}_{M}^{\varepsilon, n}(v)$ defined by (154) satisfies the estimate:

$$
\left|\mathcal{I}_{M}^{\varepsilon, n}(v)\right| \leqslant C_{\alpha, n} \varepsilon^{\frac{1}{2}} \varepsilon^{\alpha n}\|v\|_{H^{1}\left(\Omega_{\varepsilon}^{\mu}\right)} .
$$

Proof. In an obvious manner, each open set $\mathcal{O}_{j, \varepsilon}^{\mu}:=\operatorname{supp} \nabla \chi_{j}^{\varepsilon} \cap \Omega_{\varepsilon}^{\mu,-}$ can be decomposed as (Fig. 8),

$$
\mathcal{O}_{j, \varepsilon}^{\mu}=\mathcal{O}_{j, \varepsilon}^{\mu,+} \cup \mathcal{O}_{j, \varepsilon}^{\mu,-} \cup \mathcal{O}_{j, \varepsilon}^{\mu, 0}
$$

so that we can decompose $\mathcal{I}_{\mathrm{M}, \mathrm{q}}^{\varepsilon, n}(v), q=1,2$ accordingly (with obvious definitions non explicited here)

$$
\mathcal{I}_{\mathrm{M}, \mathrm{q}}^{\varepsilon, n}(v)=\mathcal{I}_{\mathrm{M}, \mathrm{q},+}^{\varepsilon, n}(v)+\mathcal{I}_{\mathrm{M}, \mathrm{q},-}^{\varepsilon, n}(v)+\mathcal{I}_{\mathrm{M}, \mathrm{q}, 0}^{\varepsilon, n}(v), \quad q=1,2 .
$$

Estimate of $\mathcal{I}_{\mathbf{M}, \mathbf{1}}^{\varepsilon, n}(v)$. We explain how to estimate $\mathcal{I}_{\mathrm{M}, 1,+}^{\varepsilon, n}(v)$, the other terms are treated similarly. We have

$$
\mathcal{I}_{\mathrm{M}, 1,+}^{\varepsilon, n}(v)=\sum_{j \in \mathbb{Z}} \mathcal{I}_{\mathrm{M}, 1,+}^{\varepsilon, j, n}(v), \quad \mathcal{I}_{\mathrm{M}, 1,+}^{\varepsilon, j, n}(v):=\int_{\mathcal{O}_{j, \varepsilon}^{\mu,+}} \nabla \chi_{j}^{\varepsilon} \cdot \nabla v\left(u_{j+\frac{1}{2}}^{\varepsilon, n}-U_{j}^{\varepsilon, n}\right) .
$$

By Cauchy-Schwartz inequality,

$$
\forall j \in \mathbb{Z}, \quad\left|\mathcal{I}_{\mathrm{M}, 1,+}^{\varepsilon, j, n}(v)\right| \leqslant\left\|u_{j+\frac{1}{2}}^{\varepsilon, n}-U_{j}^{\varepsilon, n}\right\|_{L^{\infty}\left(\mathcal{O}_{j, \varepsilon}^{\mu,+}\right)}\left\|\nabla \chi_{j}^{\varepsilon}\right\|_{L^{2}\left(\mathcal{O}_{j, \varepsilon}^{\mu,+}\right)}\|\nabla v\|_{L^{2}\left(\mathcal{O}_{j, \varepsilon}^{\mu,+}\right)}
$$

According to (145) and (148), we have

$$
\left[u_{j+\frac{1}{2}}^{\varepsilon, n}-U_{j}^{\varepsilon, n}\right](s, y)=\sum_{k=0}^{n} \varepsilon^{k}\left(u_{j+\frac{1}{2}}^{(k)}(s)-U_{j}^{(k)}\left(\frac{s}{\varepsilon}, \frac{y+L / 2}{\varepsilon}\right)\right) .
$$

Next, we use for $u_{j+\frac{1}{2}}^{(k)}$ (resp.) $U_{j}^{(k)}$ a Taylor expansion (at order $n-k$ ) (resp. the modal expansion (43)).

With the information about the $j$-dependence of these functions (see Section 4), we can write

$$
\left\{\begin{array}{l}
u_{j+\frac{1}{2}}^{(k)}(s)=\sum_{\ell=0}^{n-k} \partial_{s}^{\ell} u_{j+\frac{1}{2}}^{(k)}(0) \frac{s^{\ell}}{\ell !}+\delta u_{j+\frac{1}{2}}^{(k, n)}(s), \quad\left|\delta u_{j+\frac{1}{2}}^{(k, n)}(s)\right| \leqslant C_{k, n}\langle j\rangle^{k}|r|^{j}|s|^{n-k+1}, \\
U_{j}^{(k)}\left(\frac{s}{\varepsilon}, \frac{y+L / 2}{\varepsilon}\right)=p_{j, \pm}^{(k)}\left(\frac{s}{\varepsilon}\right)+\delta U_{j}^{(k)}\left(\frac{s}{\varepsilon}, \frac{y+L / 2}{\varepsilon}\right),\left|\delta U_{j}^{(k)}\left(\frac{s}{\varepsilon}, \frac{y+L / 2}{\varepsilon}\right)\right| \leqslant C_{k, n}\langle j\rangle^{k}|r|^{j} e^{-\pi \frac{|s|}{\varepsilon}},
\end{array}\right.
$$

where $\langle j\rangle:=\left(1+j^{2}\right)^{\frac{1}{2}}$, the $C_{k, n}$ 's are positive constants independent of $j$ and $|r|<1$. 
From the matching conditions (54), we recall that $p_{j,+}^{(k)}(s)=\sum_{\ell=0}^{k} \partial_{s}^{\ell} u_{j+\frac{1}{2}}^{(k-\ell)}(0) \frac{s^{\ell}}{\ell !}$.

With this property, it is easy to check (details are left to the reader) that the following equality holds (as a matter of fact, it suffices more or less to reproduce the computations of Section 2.3, where the matching conditions have been precisely built in order that this equality holds)

$$
\sum_{k=0}^{n} \varepsilon^{k} \sum_{\ell=1}^{n-k} \partial_{s}^{\ell} u_{j+\frac{1}{2}}^{(k)}(0) \frac{s^{\ell}}{\ell !} \equiv \sum_{k=0}^{n} \varepsilon^{k} p_{j, \pm}^{(k)}\left(\frac{s}{\varepsilon}\right) .
$$

Exploiting this equality, we deduce from (158) and (159) that

$$
\left|u_{j+\frac{1}{2}}^{\varepsilon, n}(s, y)-U_{j}^{\varepsilon, n}(s, y)\right| \leqslant \sum_{k=0}^{n} \varepsilon^{k}\left(\left|\delta U_{j}^{(k)}\left(\frac{s}{\varepsilon}, \frac{y+L / 2}{\varepsilon}\right)\right|+\left|\delta u_{j+\frac{1}{2}}^{(k, n)}(s)\right|\right) .
$$

Therefore, since, in $\mathcal{O}_{j, \varepsilon}^{\mu,+}, \varepsilon^{\alpha} \leqslant s=x-j \leqslant 2 \varepsilon^{\alpha}$, we deduce from (159) that, with $C_{n}:=\max _{k \leqslant n} C_{k, n}$,

$$
\left\|u_{j+\frac{1}{2}}^{\varepsilon, n}-U_{j}^{\varepsilon, n}\right\|_{L^{\infty}\left(\mathcal{O}_{j, \varepsilon}^{\mu,+}\right)} \leqslant C_{n}\langle j\rangle^{n}|r|^{j} \sum_{k=0}^{n} \varepsilon^{k}\left[\left(2 \varepsilon^{\alpha}\right)^{(n-k+1)}+e^{-\pi \varepsilon^{\alpha-1}}\right] .
$$

Since $\alpha<1$, we can "forget" the term $e^{-\pi \varepsilon(\alpha-1)}$ and get, using $\min _{0 \leqslant k \leqslant n}[\alpha(n-k+1)+k]=(n+1) \alpha$,

$$
\left\|u_{j+\frac{1}{2}}^{\varepsilon, n}-U_{j}^{\varepsilon, n}\right\|_{L^{\infty}\left(\mathcal{O}_{j, \varepsilon}^{\mu,+}\right)} \leqslant C_{\alpha, n}\langle j\rangle^{n}|r|^{j} \varepsilon^{\alpha(n+1)}
$$

where $C_{\alpha, n}$ blows up when $\alpha \rightarrow 1$. On the other hand, since $\left|\nabla \chi_{j}^{\varepsilon}\right| \leqslant C \varepsilon^{-\alpha}$, we observe that

$$
\left\|\nabla \chi_{j}^{\varepsilon}\right\|_{L^{2}\left(\mathcal{O}_{j, \varepsilon}^{\mu,+}\right)} \leqslant C \varepsilon^{-\alpha} \operatorname{meas}\left(\mathcal{O}_{j, \varepsilon}^{\mu,+}\right)^{\frac{1}{2}} \leqslant C \varepsilon^{\frac{1-\alpha}{2}} .
$$

Finally, substituting (160) and (161) in (156) and (157), and using the discrete Cauchy-Schwartz inequality (for the sum over $j$ ), we obtain

$$
\begin{gathered}
|| \mathcal{I}_{\varepsilon, n}^{\mathrm{M}, 1,+}(v) \mid \leqslant C_{\alpha, n} \varepsilon^{\alpha\left(n+\frac{1}{2}\right)} \varepsilon^{\frac{1}{2}}\left(\sum_{j \in \mathbb{Z}}\langle j\rangle^{2 n}|r|^{2 j}\right)^{\frac{1}{2}}\left(\sum_{j \in \mathbb{Z}}\|\nabla v\|_{L^{2}\left(\mathcal{O}_{j, \varepsilon}^{\mu,+}\right)}^{2}\right)^{\frac{1}{2}} \\
\leqslant C_{\alpha, n} \varepsilon^{\alpha\left(n+\frac{1}{2}\right)} \varepsilon^{\frac{1}{2}}\|\nabla v\|_{L^{2}\left(\Omega_{\varepsilon}^{\mu}\right)} .
\end{gathered}
$$

Estimate of $\mathcal{I}_{\mathbf{M}, 2}^{\varepsilon, n}(v)$. We only treat $\mathcal{I}_{\mathrm{M}, 2,+}^{\varepsilon, n}(v)$ and point out the difference with $\mathcal{I}_{\mathrm{M}, 1,+}^{\varepsilon, n}(v)$. We have

$$
\mathcal{I}_{\mathrm{M}, 2,+}^{\varepsilon, n}(v)=\sum_{j \in \mathbb{Z}} \mathcal{I}_{\mathrm{M}, 2,+}^{\varepsilon, j, n}(v), \quad \mathcal{I}_{\mathrm{M}, 2,+}^{\varepsilon, j, n}(v):=-\int_{\mathcal{O}_{j, \varepsilon}^{\mu,+}} \nabla \chi_{j}^{\varepsilon} \cdot \nabla\left(u_{\mathrm{FF}}^{\varepsilon, n}-U_{j}^{\varepsilon, n}\right) v .
$$


Then, we estimate each $\mathcal{I}_{\mathrm{M}, 2,+}^{\varepsilon, j, n}(v)$ as

$$
\forall j \in \mathbb{Z}, \quad\left|\mathcal{I}_{\mathrm{M}, 2,+}^{\varepsilon, j, n}(v)\right| \leqslant\left\|\nabla\left(u_{j+\frac{1}{2}}^{\varepsilon, n}-U_{j}^{\varepsilon, n}\right)\right\|_{L^{\infty}\left(\mathcal{O}_{j, \varepsilon}^{\mu,+}\right)}\left\|\nabla \chi_{j}^{\varepsilon}\right\|_{L^{2}\left(\mathcal{O}_{j, \varepsilon}^{\mu,+}\right)}\|v\|_{L^{2}\left(\mathcal{O}_{j, \varepsilon}^{\mu,+}\right)}
$$

Similarly to (160), we prove that (the reader will note that, with respect to (160), we loose an $\varepsilon^{\alpha}$ term because we have to differentiate the formulas in (159))

$$
\left\|\nabla\left(u_{j+\frac{1}{2}}^{\varepsilon, n}-U_{j}^{\varepsilon, n}\right)\right\|_{L^{\infty}\left(\mathcal{O}_{j, \varepsilon}^{\mu,+}\right)} \leqslant C_{\alpha, n}\langle j\rangle^{n}|r|^{j} \varepsilon^{\alpha n}
$$

Therefore, prodceeding as for obtaining (162), we get

$$
\left|\mathcal{I}_{\mathrm{M}, 2,+}^{\varepsilon, n}(v)\right| \leqslant C_{\alpha, n} \varepsilon^{\alpha\left(n-\frac{1}{2}\right)} \varepsilon^{\frac{1}{2}}\left(\sum_{j \in \mathbb{Z}}\|v\|_{L^{2}\left(\mathcal{O}_{j, \varepsilon}^{\mu,+}\right)}^{2}\right)^{\frac{1}{2}}
$$

To pursue, we use the following estimate, that aims at exploiting the smallness of the domains $\mathcal{O}_{j, \varepsilon}^{\mu,+}$ and the $H^{1}$ regularity of $v$

$$
\left(\sum_{j \in \mathbb{Z}}\|v\|_{L^{2}\left(\mathcal{O}_{j, \varepsilon}^{\mu,+}\right)}^{2}\right)^{\frac{1}{2}} \leqslant C_{\alpha} \varepsilon^{\frac{\alpha}{2}}\|v\|_{H^{1}\left(\Omega_{\varepsilon}^{\mu}\right)} .
$$

The proof of this estimate is easily deduced from the following lemma:

Lemma 8. Let $Q:=(0, a) \times(0, b)$ and $Q_{\eta}=I_{\eta} \times(0, b) \subset Q$ with meas $I_{\eta}=\eta$, then there exists $C>0$ independent of $\eta$ such that

$$
\forall Q_{\eta} \subset Q, \quad \forall v \in H^{1}(Q), \quad\|v\|_{L^{2}\left(Q_{\eta}\right)} \leqslant C \eta^{\frac{1}{2}}\|v\|_{H^{1}\left(Q_{\eta}\right)}
$$

Proof. Let $v$ be smooth enough, from the embedding $H^{1}(0, a) \subset L^{\infty}(o, a)$, we have

$$
\forall(x, y) \in Q, \quad|u(x, y)|^{2} \leqslant C \int_{0}^{a}\left(\left|\partial_{x} u(\xi, y)\right|^{2}+|u(\xi, y)|^{2}\right) d \xi
$$

We obtain (168) by integrating the above inequality over $Q_{\eta}$ and conclude with a density argument.

Using (167), we conclude from (166) that

$$
\left|\mathcal{I}_{\mathrm{M}, 2,+}^{\varepsilon, n}(v)\right| \leqslant C_{\alpha, n} \varepsilon^{\frac{1}{2}} \varepsilon^{\alpha n}\|v\|_{H^{1}\left(\Omega_{\varepsilon}^{\mu}\right)} .
$$

Regrouping the estimates (162) and (169), and their equivalent for $\mathcal{I}_{\mathrm{M}, 1, \delta}^{\mathcal{E}, n}(v)$ and $\mathcal{I}_{\mathrm{M}, 2, \delta}^{\varepsilon, n}(v), \delta \in\{-, 0\}$, we obtain the result of Lemma 7 (note that (169) is worse than (162)). 
5.3. Estimation of the consistency errors.

Lemma 9. The consistency errors defined in (154) satisfy the estimates

(i) $\left|\mathcal{I}_{F F}^{\varepsilon, n}(v)\right| \leqslant C_{n} \varepsilon^{n+\frac{3}{2}}\|v\|_{L^{2}\left(\Omega_{\varepsilon}^{\mu}\right)} \quad$ (ii) $\quad\left|\mathcal{I}_{N F}^{\varepsilon, n}(v)\right| \leqslant C_{n} \varepsilon^{(n+1) \alpha-\frac{1}{2}}\|v\|_{H^{1}\left(\Omega_{\varepsilon}^{\mu,-}\right)}$.

Proof. By definition (see (154)) of $\mathcal{I}_{\mathrm{FF}}^{\varepsilon, n}(v)$ and Cauchy-Schwartz inequality, we get, since $\left|1-\chi^{\varepsilon}\right| \leqslant 1$,

$$
\left|\mathcal{I}_{\mathrm{FF}}^{\varepsilon, n}(v)\right| \leqslant C_{n} \varepsilon^{n+1}\left\|r_{\mathrm{FF}}^{\varepsilon, n}\right\|_{L^{2}\left(\Omega_{\varepsilon}^{\mu}\right)}\|v\|_{L^{2}\left(\Omega_{\varepsilon}^{\mu}\right)} .
$$

According to the definition (147) of $r_{\mathrm{FF}}^{\varepsilon, n}$, we see that $\left\|r_{\mathrm{FF}}^{\varepsilon, n}\right\|_{L^{2}\left(\Omega_{\varepsilon}^{\mu,-}\right)} \leqslant C_{n} \varepsilon^{1 / 2}$ which yields (170)-(i).

In the same way, using the definition (see (154)) of $\mathcal{I}_{\mathrm{NF}}^{\varepsilon, n}(v)$ as well as continuous and discrete CauchySchwartz inequalities, we have, since $\left|\chi^{\varepsilon}\right| \leqslant 1$,

$$
\left|\mathcal{I}_{\mathrm{NF}}^{\varepsilon, n}(v)\right| \leqslant C_{n} \varepsilon^{n-1}\left(\sum_{j \in \mathbb{Z}}\left\|R_{j}^{\varepsilon, n}\right\|_{L^{2}\left(\operatorname{supp} \chi_{j, \varepsilon}\right)}^{2}\right)^{\frac{1}{2}}\left(\sum_{j \in \mathbb{Z}}\|v\|_{L^{2}\left(\operatorname{supp} \chi_{j, \varepsilon}\right)}^{2}\right)^{\frac{1}{2}}
$$

The most technical step for the estimation of the near field consistency error lies in the following lemma

Lemma 10. One has the estimate

$$
\left\|R_{j}^{\varepsilon, n}\right\|_{L^{2}\left(\text { supp } \chi_{j, \varepsilon}\right)} \leqslant C_{n}\langle j\rangle^{n}|r|^{j} \varepsilon^{\left(n+\frac{1}{2}\right)(\alpha-1)+1} .
$$

Proof. By definition (see (150)) of $R_{j}^{\varepsilon, n}$ and since $\varepsilon<1$

$$
\left\|R_{j}^{\varepsilon, n}\right\|_{L^{2}\left(\operatorname{supp} \chi_{j, \varepsilon}\right)} \leqslant \sum_{p=n-1}^{2 n} \varepsilon^{p-(n-1)}\left\|R_{j}^{p, n}\right\|_{L^{2}\left(\operatorname{supp} \chi_{j, \varepsilon}\right)} \leqslant(n+2) \sup _{n-1 \leqslant p \leqslant 2 n}\left\|R_{j}^{p, n}\right\|_{L^{2}\left(\operatorname{supp} \chi_{j, \varepsilon}\right)}
$$

We use $\operatorname{supp} \chi_{j}^{\varepsilon}=\mathcal{K}_{j}^{\varepsilon,-} \cup \mathcal{Q}_{j,+}^{\varepsilon} \cup \mathcal{Q}_{j,-}^{\varepsilon} \cup \mathcal{Q}_{j, 0}^{\varepsilon}$ (see Figure 8). Using the decay properties in $j$ of the $U_{j}^{(\ell)}$, s (see Section 4), after rescaling, we get

$$
\int_{\mathcal{K}_{j}^{\varepsilon,-}}\left|R_{j}^{p, n}\left(\frac{x-j}{\varepsilon}, \frac{y+L / 2}{\varepsilon}\right)\right|^{2} d x d y=\varepsilon^{2} \int_{K_{j}}\left|R_{j}^{p, n}\right|^{2} \leqslant C_{n}\langle j\rangle^{2 n}|r|^{2 j} \varepsilon^{2} .
$$

On the other hand, using the same change of variable, we have

$$
\int_{\mathcal{Q}_{j,+}^{\varepsilon}}\left|R_{j}^{p, n}\left(\frac{x-j}{\varepsilon}, \frac{y+L / 2}{\varepsilon}\right)\right|^{2} d x d y=\varepsilon^{2} \int_{1}^{2 \varepsilon^{\alpha-1}} \int_{0}^{1}\left|R_{j}^{p, n}(X, Y)\right|^{2} d X d Y .
$$

The estimate then relies on one hand on the exponential decay of $R_{j}^{p, n}$ with respect to $j$, on the other hand on the polynomial growth with respect to $X$ of $R_{j, p}^{n}(X, Y)$.

The formula (43) of Proposition 2, with $X$ instead of $\mathfrak{s}$, says that $U_{j}^{(\ell)}(X, Y)$ grows proportionnally to $X^{\ell}$. Thus from the definition (150) of $R_{j}^{p, n}$ and the decay propery in $j$, we infer that

$$
R_{j}^{p, n}(X, Y) \leqslant C_{n}\langle j\rangle^{n}|r|^{j}|X|^{q(n, p)}
$$


where $q(n, p)=\max \left\{\ell /(k, \ell) \in \mathcal{I}^{p, n}\right\}$, that is to say $q(p, n)=n$ for $p \geqslant n$ and $q(p, n)=n-1$ for $p=n-1$. Therefore, we obtain from (176), since $|X| \leqslant 2 \varepsilon^{\alpha-1}$ in the integral at the right hand side,

$$
\left\{\begin{array}{l}
\int_{\mathcal{Q}_{j,+}^{\varepsilon}}\left|R_{j}^{p, n}\left(\frac{x-j}{\varepsilon}, \frac{y+L / 2}{\varepsilon}\right)\right|^{2} \leqslant C_{n}\langle j\rangle^{2 n}|r|^{2 j} \varepsilon^{2} \varepsilon^{\alpha-1} \varepsilon^{2(\alpha-1) n}, \quad \text { for } n \leqslant p \leqslant 2 n, \\
\int_{\mathcal{Q}_{j,+}^{\varepsilon}}\left|R_{j}^{n-1, n}\left(\frac{x-j}{\varepsilon}, \frac{y+L / 2}{\varepsilon}\right)\right|^{2} \leqslant C_{n}\langle j\rangle^{2 n}|r|^{2 j} \varepsilon^{2} \varepsilon^{\alpha-1} \varepsilon^{2(\alpha-1)(n-1)}
\end{array}\right.
$$

Here, we have used that meas (] $1,2 \varepsilon^{\alpha-1}[\times] 0,1[) \leqslant 2 \varepsilon^{\alpha-1}$. Comparing (175) with (177) (and corresponding estimates for the integrals over $\mathcal{Q}_{j,-}^{\varepsilon}$ and $\mathcal{Q}_{j, 0}^{\varepsilon}$ ), and retaining the smallest power of $\varepsilon$ in the corresponding right hand sides, which corresponds to (177)(a) since $\alpha<1$, we get

$$
\left.\left\|R_{j}^{p, n}\right\|_{L^{2}(\text { supp } \chi j, \varepsilon}\right) \leqslant C_{n}\langle j\rangle^{n}|r|^{j} \varepsilon^{\left(n+\frac{1}{2}\right)(\alpha-1)+1}
$$

Substituting these estimates into (174) leads to the announced result.

Subtituting (173) in (172) then gives $\left|\mathcal{I}_{\mathrm{NF}}^{\varepsilon, n}(v)\right| \leqslant C_{n} \varepsilon^{\left(n+\frac{1}{2}\right) \alpha-\frac{1}{2}}\left(\sum_{j \in \mathbb{Z}}\|v\|_{L^{2}\left(\operatorname{supp} \chi_{j}^{\varepsilon}\right)}^{2}\right)^{\frac{1}{2}}$.

Finally, since, using again Lemma 8, we have

$$
\left(\sum_{j \in \mathbb{Z}}\|v\|_{L^{2}\left(\operatorname{supp} \chi_{j}^{\varepsilon}\right)}^{2}\right)^{\frac{1}{2}} \leqslant C_{\alpha} \varepsilon^{\frac{\alpha}{2}}\|v\|_{H^{1}\left(\Omega_{\varepsilon}^{\mu,-}\right)},
$$

we deduce the estimate (170)-(ii).

\subsection{Completion of the proof of Theorem 1}

Using (with $m$ instead of $n$ ) the estimates (155) (Lemma 7) and (170) (Lemma 9), we deduce from (153) (with $m$ instead of $n$ ) that the estimate (19) holds with $\alpha_{m}=\alpha m+\alpha-\frac{1}{2}$. According to Lemma 1, the proof of theorem 1 is thus complete.

\section{A numerical approach based on asymptotics expansions.}

\subsection{Description of the method}

Following the iterative construction described in Section 4, we derive a numerical method to compute successively the terms of the asymptotic expansion (up to a given order prescribed by the user). Let us give here the main steps of the algorithm, that relies of course about an initial choice of the limit eigenvalue $\lambda^{(0)}$ :

A- Initialization step : $k=0$

1. Pick a $\lambda^{(0)}$ by solving numerically (via a Newton method) equation (9). 
2. Explicit construction of $u^{(0)}$ by formulas (93).

3. Construction of $U^{(0)}$ using formula (94).

B- Construction of the terms of order $k, k \in \mathbb{N}^{*}$

0. Preliminary computations of

(a) the quantities $\hat{g}_{\delta, \ell}^{(k-1)}$ and $\hat{\Phi}_{\ell}^{(k-2)}, 0 \leqslant \ell \leqslant k-1$ so that $g_{j, \delta}^{(k-1)}$ and $\Phi_{j}^{(k-2)}$ defined by (68) and (65) respectively sasitfy (125-126) .

(b) the quantities $\Delta_{+, 0}^{(k-1)}, \Xi_{0}^{(k-1)}$ via (77) for $j=0$.

(c) the polynomials $\hat{\Xi}^{(k-1)}(\cdot), \hat{\Delta}_{ \pm}^{(k-1)}(\cdot)$ so that (128) holds for $\Delta_{ \pm, 0}^{(k-1)}, \Xi_{0}^{(k-1)}$ defined by (77).

1. Computation of $\lambda^{(k)}$ using Formula (88).

2. Explicit determination of $u^{(k)}$.

(a) Computation of the polynomials $a_{\ell}^{(k)}(\cdot), b_{\ell}^{(k)}(\cdot), c_{\ell}^{(k)}(\cdot)$ and $d_{\ell}^{(k)}(\cdot), 1 \leqslant \ell \leqslant k$ in order that Formulas (130) provide a particular solution of (85).

(b) Computation of the coefficients $c_{\ell, 0}^{(k)}$ and $d_{\ell, 0}^{(k)}, 1 \leqslant \ell \leqslant k$ in order that (120) holds $u_{0 \text {,part }}^{(k)}$.

(c) Computation of the polynomials $b_{k}(\cdot)$ and $c_{k}(\cdot)$ in order that the equations (85)-(v) become (133) taking (131) into account. This can be done from step (a) (see (134)).

(d) Computation of the polynomial $d_{0}^{(k)}(\cdot)$ using (132). This can also be done from step (a).

(e) Computation of the coefficients $\alpha_{\ell}^{(k)}, 1 \leqslant \ell \leqslant k$ in order that $\check{a}_{\text {part }}^{(k)}(j)$ defined by (136) be a particular solution of (135).

(f) Computation of the polynomial $a_{0}^{(k)}(\cdot)$ via (137) with $\alpha_{0}^{(k)}=0$ (see (139)) .

(g) Computation of the polynomials $b_{0}^{(k)}(\cdot)$ and $c_{0}^{(k)}(\cdot)$ from (138).

(h) Computation of $c_{0,0}^{(k)} \equiv \check{c}_{0,0}^{(k)}$ from (85)-(iv) for $j=0$.

3. Semi-Explicit determination of $U^{(k)}$.

(a) Numerical determination of $U_{0}^{(k)}$ associated to the perturbed junction.

(b) Explicit computation of the polynomial $\mathcal{P}^{(k)}(j)$ in order to satisfy (118).

(c) Numerical determination of the $k$ profile functions $\mathcal{U}_{\ell}^{(k)}$ solution of (143).

(d) Computation of $U_{j}^{(k)}$ for $j \in \mathbb{N}^{*}$ using formula (121).

As already mentioned, in the above algorithm, except for points B-3.(a) and B-3.(c), all the steps are achieved through hand computations that can be found detailed in [27]. At stage $k$, the steps B-3.(a) and B-3.(c) require finite element computations, requiring two meshes, one of the rectangle $K_{0}$, one of the rectangle $K_{1}$, for the solutions of problems (118). Another approximation parameter is the truncation order $N$ of the series in the definition of the DtN operators $T_{\delta}, \delta=0, \pm$. Even though the finite element calculations of $U_{0}^{(k)}$ (resp. the profiles $\mathcal{U}_{\ell}^{(k)}$ ) are done inside the rectangle $K_{0}$ they can be extended analytically, as in the proof of Proposition 4, in the whole junction $\mathcal{J}_{0}\left(\mathcal{J}_{1}\right)$ up to the same series truncation 
issue as for the DtN operators. Once the meshes and $N$ has been chosen, we only have two (symmetric positive definite) finite element matrices to be inverted : $\mathbf{A}_{0}$ (for $K_{0}$ ) and $\mathbf{A}_{1}$ (for $K_{1}$ ), of which a Cholesky factorization can be done at the beginning of the algorithm. At stage $k$, we have to solve

- one linear system with $\mathbf{A}_{0}$, for computing $U_{0}^{(k)}$

- $k+1$ linear system with $\mathbf{A}_{1}$ (and different right hand sides) for computing $\mathcal{U}_{\ell}^{(m)}, 0 \leqslant m \leqslant k$

\subsection{Numerical results}

In the following section, we choose $L=2$. In that case, the essential spectrum of the limit operator $\mathcal{A}_{s}^{\mu}$ is given by (see Proposition 4 and Figure 9 in [1])

$$
\sigma_{\text {ess }}\left(\mathcal{A}_{s}^{\mu}\right)=\left\{\lambda=\omega^{2} \in \mathbb{R}^{+}, \omega \in \bigcup_{k \in \mathbb{N}} I_{k}\right\} \text { where } I_{k}=\left[-\arccos \left(\frac{1}{3}\right)+k \pi, \arccos \left(\frac{1}{3}\right)+k \pi\right] \forall k \in \mathbb{N}
$$

while, for any $\mu<1$, the discrete spectrum of $\mathcal{A}_{s}^{\mu}$ is

$$
\sigma_{d}\left(\mathcal{A}_{s}^{\mu}\right)=\left\{\lambda=\omega^{2}, \omega \in \bigcup_{k \in \mathbb{N}}\left\{\lambda_{*}(\mu)+k \pi,-\lambda_{*}(\mu)+k \pi\right\}\right\}
$$

where $\lambda_{*}(\mu)$ denotes the unique root of the equation (9-right) in $\left[0, \frac{\pi}{2}\right]$. To obtain $\sigma_{d}\left(\mathcal{A}_{s}^{\mu}\right)$, we used Theorem 1 in [1] ensuring that $\mathcal{A}_{s}^{\mu}$ has exactly two eigenvalues in each of its gaps together with the fact that if $\lambda=\omega^{2}$ is a solution of (9), both $\lambda^{\prime}=(\omega+k \pi)^{2}$ and $\lambda^{\prime \prime}=(k \pi-\omega)^{2}$, for any $k \in \mathbb{N}$ are also solutions of (9). We notice that the spectrum of $\mathcal{A}_{s}^{\mu}$ is the image by the function $x \mapsto x^{2}$ of a $\pi$-periodic subspace of $\mathbb{R}$. A graphic illustration of $\sigma\left(\mathcal{A}_{s}^{\mu}\right)$ is presented in Figure 9.

$$
\begin{aligned}
& -\omega^{2} \in \sigma_{\text {ess }}\left(\mathcal{A}_{s}^{\mu}\right) \\
& -\omega^{2} \in \sigma_{d}\left(\mathcal{A}_{s}^{\mu}\right)
\end{aligned}
$$

Fig. 9. The spectrum of $\sigma\left(\mathcal{A}_{s}^{\mu}\right)$ in the case $L=2$.

Computation of eigenvalues and numerical validation of the method. In the following experiments, we take $\mu=1 / 4$ and we focus on the first and fourth eigenvalues $\lambda_{1}=\lambda_{*}\left(\frac{1}{4}\right) \approx 1.80$ and $\lambda_{4} \approx 24.43$, located respectively in the first and second gaps of the operator $\mathcal{A}_{s}^{1 / 4}$ (see Figure 9). The associated limit eigenvectors (defined on the graph by (24-25)) are represented on Figure 10.

The numerical results associated with the first eigenvalue $\lambda_{1}$ are represented on Figure 11. In the left part, we compute the evolution of

$$
\lambda^{\varepsilon, n}=\sum_{k=0}^{n} \varepsilon^{k} \lambda^{(k)}
$$




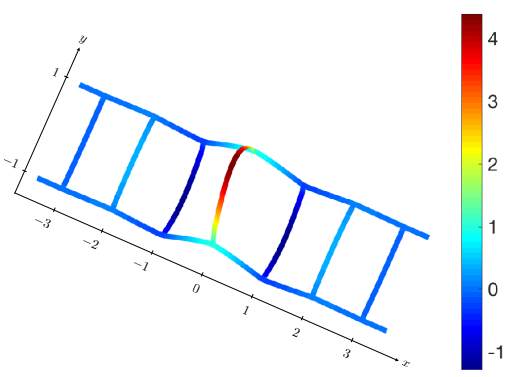

(a) $\lambda_{1}$

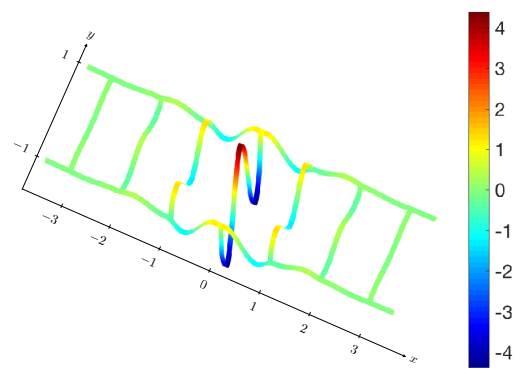

(b) $\lambda_{4}$

Fig. 10. Representation of two limit eigenvectors associated with $\lambda_{1}$ and $\lambda_{2}$

with respect to $\varepsilon$ for $n$ varying between 1 and 5 and $\varepsilon$ between 0.02 and 0.6 . To compute the near field terms (part 3-(c) of the algorithm described in Section 6.1), we first truncate the junctions $\mathcal{J}_{0}$ and $\mathcal{J}_{1}$ at a distance $T=5$ and we use a first order approximation of the Dirichlet-to-Neumann operator. The problem is then numerically solved by a $P_{1}$-finite element method using a uniform mesh of mesh-size $h=0.002$. We compare $\lambda^{\varepsilon, n}$ with a reference value of $\lambda^{\varepsilon}$ obtained by computing numerically the first eigenvalue of the full two dimensional operator $A_{\varepsilon, s}^{\mu}$ using the method developed in [28]. In a nutshell, this method permits us to rewrite the initial eigenvalue problem, posed in the unbounded domain $\Omega_{\varepsilon}^{\mu}$, as a non linear eigenvalue problem posed in a bounded domain. This is done by computing the (approximate) Dirichlet-to-Neumann operators for periodic domains (see [29,30]), which requires to solve periodic cell problems (discretized here again using the standard $P_{1}$ finite element methods) and a stationary Ricatti equation. By contrast to the initial problem, the reduced problem (posed in a bounded domain) is a non linear eigenvalue problem (since the DtN operators depend on the eigenvalue) of a fixed point nature. It is solved using a Newton-type procedure, each iteration needing a finite element computation, see [28] for more details.

We notice that the approximation of $\lambda^{\varepsilon}$ by $\lambda^{\varepsilon, n}$ is qualitatively good, especially when adding high order terms in the truncated series (179). Surprisingly, the approximation remains accurate even for a rather large $\varepsilon$ (the geometry of the domain $\Omega_{\varepsilon}^{\mu}$ for $\varepsilon=0.6$ does not really looks like a graph-like structure).

To verify the accuracy of our asymptotic expansion, we represent on Figure 11 the evolution of the errors $e_{n}=\left|\lambda^{\varepsilon, n}-\lambda^{\varepsilon}\right|$ with respect to $\varepsilon, \varepsilon$ varying between 0.02 and 0.6 . For the first two orders, the experimental convergence rates (2.1 for $e_{1}$ and 2.9 for $e_{2}$ ) coïncide with the theoretical ones. Unfortunately, this is not the case for the higher order ones. It might be due to the fact that the 'exact' solution $\lambda^{\varepsilon}$ is computed with a limited precision of $10^{-3}$. The use of a second order finite element method for the different numerical computations may confirm this point but is beyond the scope of this paper.

The same experiment is reproduced for the fourth eigenvalue $\left(\lambda_{4} \approx 24.43\right)$ on Figure 12 . Here again, the approximation of $\lambda^{\varepsilon}$ by $\lambda^{\varepsilon, n}$ is qualitatively good, especially for high orders. However, we observe that for a given $\varepsilon$ the error is bigger for $\lambda_{4}$ than for $\lambda_{1}$. We point out that we are not able to compute $\lambda^{\varepsilon}$ for $\varepsilon>0.25$. In that case, we do not know if the eigenvalue is close to the essential spectrum or does not 

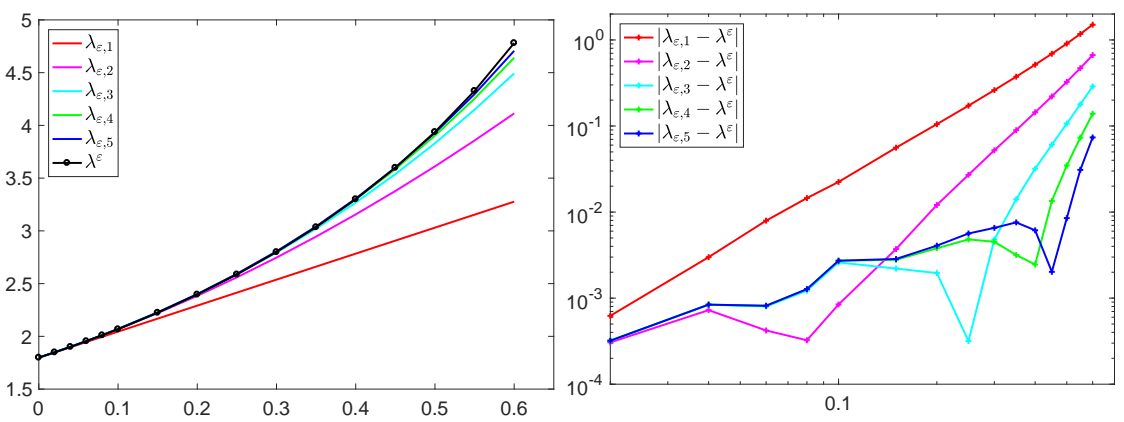

Fig. 11. Results for the first eigenvalue: $\lambda^{\varepsilon, 1}$ w.r.t $\varepsilon$ (left), Error w.r.t $\varepsilon$ (right).
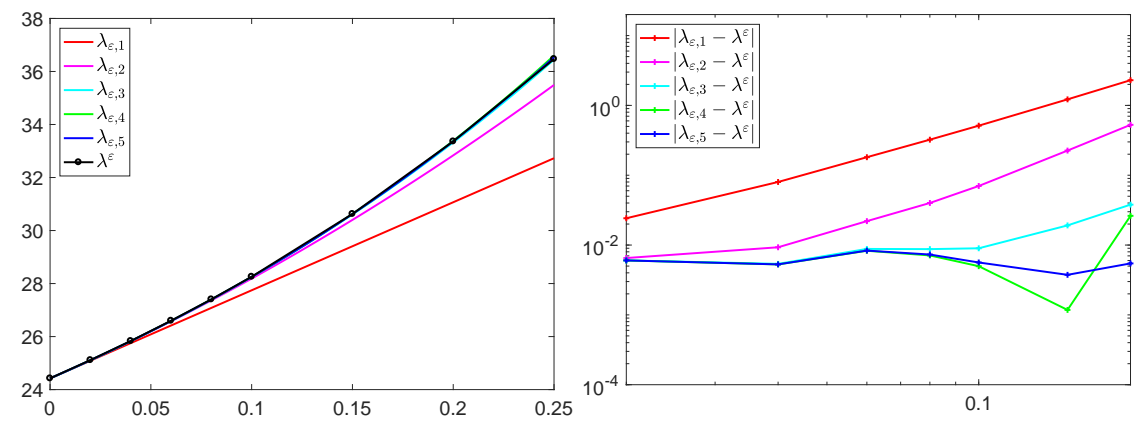

Fig. 12. Results for the fourth eigenvalue:: $\lambda^{\varepsilon, 4}$ w.r.t $\varepsilon$ (left), Error w.r.t $\varepsilon$ (right).

exist anymore.

To summarize, from a computational point of view, the main advantage of the asymptotic method is that it suffices to make one computation in order to obtain an approximation of $\lambda^{\varepsilon}$ for an arbitrary value $\varepsilon$. Moreover, the approximation is highly-accurate when $\varepsilon$ is small (the accuracy depending of the numerical error made in the computation of the near field terms). Nevertheless, by nature, the asymptotic method fails to predict the possible disparition of the eigenvalue into the essential spectrum as $\varepsilon$ becomes large. In that case, a high order direct method would be preferable (see e.g [31]).

Example of near field terms. To end this part, let us give a few examples of near field terms obtained when computing the first eigenvalue $\lambda_{1}$ (see Fig 9). We shall focus on the junctions $\mathcal{J}_{0}(j=0$, perturbed junction) and $\mathcal{J}_{1}(\mathrm{j}=1$, first unmodified junction) as represented on Figure 13.

In Figure 14, we display the near fields terms $U_{0}^{(1)}$ and $U_{0}^{(2)}$, that is to say the near field terms of order 1 and 2 in the junction $\mathcal{J}_{0}$. A zoom on the central part of the junction is represented on the right part of the two pictures. We remark that these two fields are symmetric with respect to $x=0$. Moreover, they tend to grow inside the branches of the junction. To quantify this growth, we plot on Figure 15 the representative curves of the two fields along the horizontal cut $y=1 / 2$ and the vertical cut $x=0$. As expected, the first near field is linearly increasing while the second one has a quadratic growth.

Then, the same near fields are represented in the junction $\mathcal{J}_{1}$ are displayed on Figures 16-17. As expected, the fields are not symmetric anymore. However, they are still polynomial growing, bur the polynomials are different in the left $(x<0)$ and right part $(x>0)$ of the junction. 


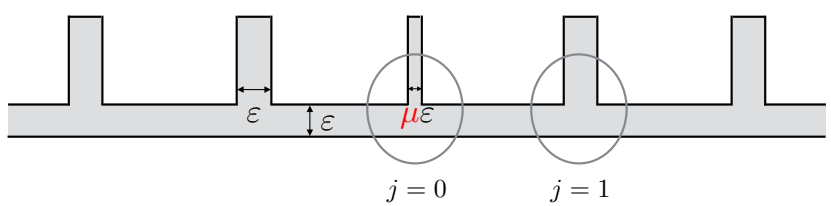

Fig. 13. Schematic representation of the domain under consideration

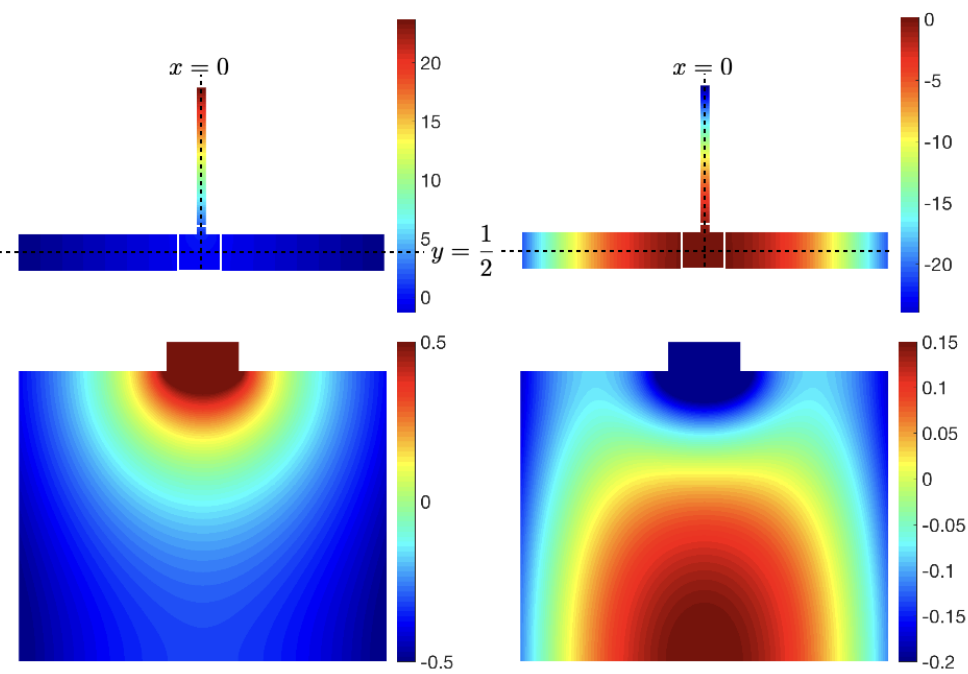

Fig. 14. Near fields $U_{0}^{(1)}$ (left) and $U_{0}^{(2)}$ (right) in $\mathcal{J}_{0}$. Bottom picture: zoom inside the white rectangle
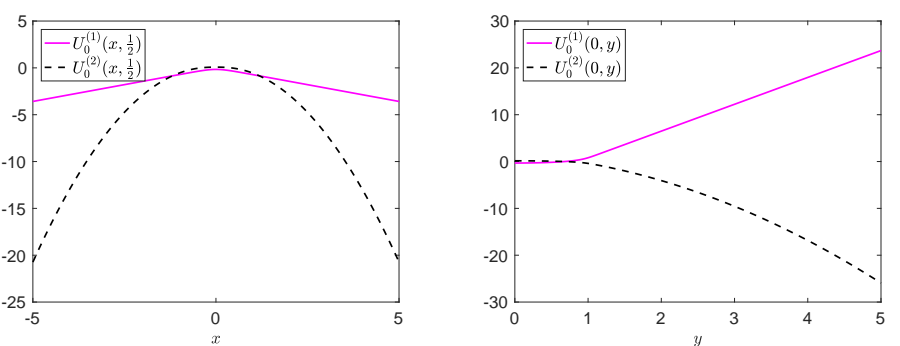

Fig. 15. Cuts of the near fields $U_{0}^{(1)}$ and $U_{0}^{(2)}$ along $y=\frac{1}{2}$ (left) and $x=0$ in $\mathcal{J}_{0}$ (right)

\section{References}

[1] B. Delourme, S. Fliss, P. Joly and E. Vasilevskaya, Trapped modes in thin and infinite ladder like domains. Part 1: existence results, Asymptot. Anal. 103(3) (2017), 103-134. doi:10.3233/ASY-171422.

[2] P. Exner, Contact interactions on graph superlattices, Journal of Physics A: Mathematical and General 29(1) (1996), 87.

[3] R. Carlson, Adjoint and self-adjoint differential operators on graphs, Electronic Journal of Differential Equations 6(1998) (1998), 1-10.

[4] P. Kuchment and H. Zeng, Convergence of spectra of mesoscopic systems collapsing onto a graph, J. Math. Anal. Appl. 258(2) (2001), 671-700. doi:10.1006/jmaa.2000.7415.

[5] P. Kuchment, Quantum graphs. I. Some basic structures, Waves Random Media 14(1) (2004), S107-S128, Special section on quantum graphs. doi:10.1088/0959-7174/14/1/014. 


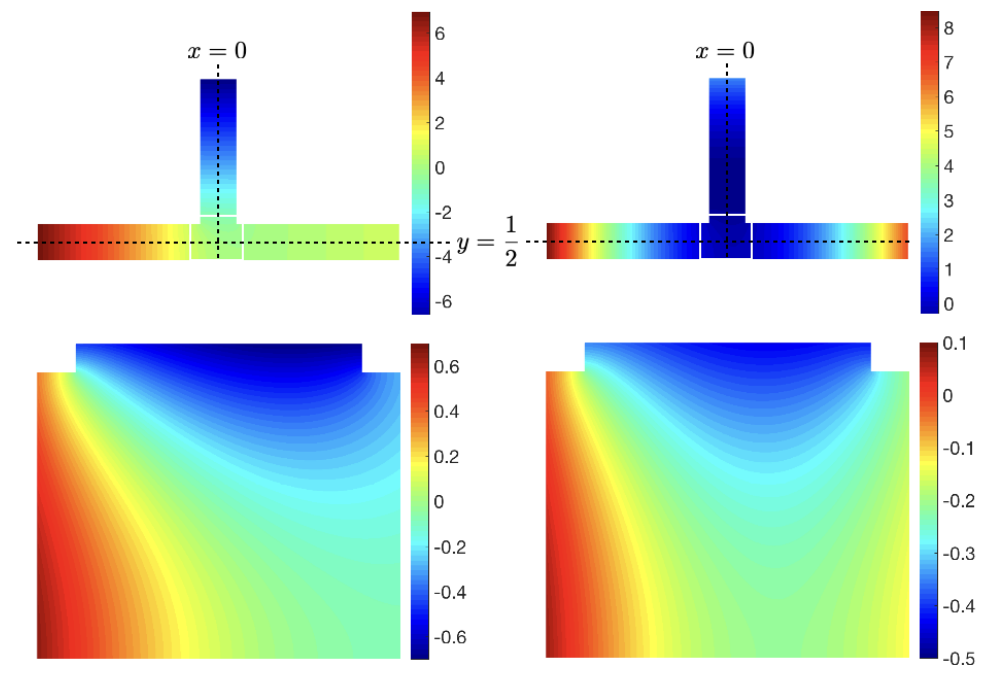

Fig. 16. Near fields $U_{1}^{(1)}$ (left) and $U_{1}^{(2)}$ (right) in $\mathcal{J}_{1}$. Bottom picture: zoom inside the white rectangle
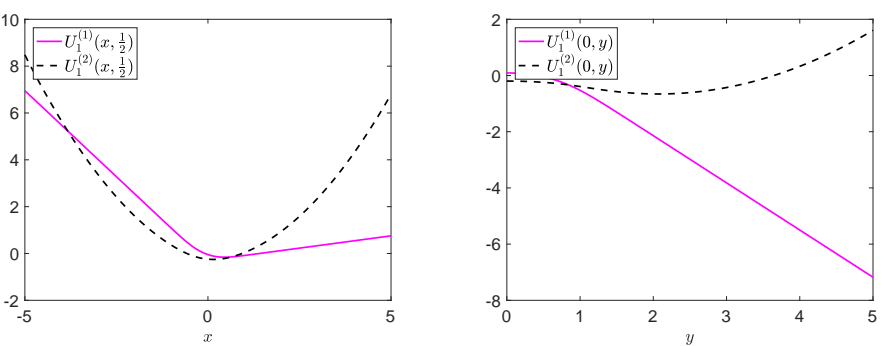

Fig. 17. Cuts of the near fields $U_{0}^{(1)}$ and $U_{0}^{(2)}$ along $y=\frac{1}{2}$ (left) and $x=0$ in $\mathcal{J}_{1}$ (right)

[6] M.S. Birman and M.Z. Solomjak, Spectral theory of selfadjoint operators in Hilbert space, Mathematics and its Applications (Soviet Series), D. Reidel Publishing Co., Dordrecht, 1987, p. xv+301, Translated from the 1980 Russian original by S. Khrushchëv and V. Peller. ISBN 90-277-2179-3.

[7] M.S.P. Eastham, The spectral theory of periodic differential equations, distributed by chatto and windus edn, Edinburgh : Scottish Academic Press, Edinburgh-London, 1973.

[8] M. Reed and B. Simon, Methods of modern mathematical physics v. I-IV, Academic Press, New York, 1972-1978.

[9] P. Kuchment, Floquet theory for partial differential equations, Operator Theory: Advances and Applications, Vol. 60, Birkhäuser Verlag, Basel, 1993, p. xiv+350. ISBN 3-7643-2901-7.

[10] O. Post, Spectral convergence of quasi-one-dimensional spaces, Ann. Henri Poincaré 7(5) (2006), 933-973. doi:10.1007/s00023-006-0272-x.

[11] V. Maz'ya, S. Nazarov and B. Plamenevskij, Asymptotic theory of elliptic boundary value problems in singularly perturbed domains. Vol. I, Operator Theory: Advances and Applications, Vol. 111, Birkhäuser Verlag, Basel, 2000, p. xxiv+435, Translated from the German by Georg Heinig and Christian Posthoff. ISBN 3-7643-6397-5.

[12] D. Grieser, Spectra of graph neighborhoods and scattering, Proc. Lond. Math. Soc. (3) 97(3) (2008), 718-752. doi: $10.1112 / \mathrm{plms} / \mathrm{pdn} 020$.

[13] S.A. Nazarov, Bounded solutions in a T-shaped waveguide and the spectral properties of the Dirichlet ladder, Comput. Math. Math. Phys. 54(8) (2014), 1261-1279. doi:10.1134/S0965542514080090.

[14] S.A. Nazarov, Opening of a gap in the continuous spectrum of a periodically perturbed waveguide, Mat. Zametki 87(5) (2010), 764-786. doi:10.1134/S0001434610050123. 
[15] B. Delourme, S. Fliss, P. Joly and E. Vasilevskaya, Trapped modes in thin and infinite ladder like domains: existence and asymptotic analysis, INRIA Research Report (2016).

[16] M. Van Dyke, Perturbation methods in fluid mechanics, Applied Mathematics and Mechanics, Vol. 8, Academic Press, New York, 1964, p. $\mathrm{x}+229$.

[17] A.M. Il'in, Matching of asymptotic expansions of solutions of boundary value problems, Translations of Mathematical Monographs, Vol. 102, American Mathematical Society, Providence, RI, 1992, p. x+281, Translated from the Russian by V. Minachin [V. V. Minakhin]. ISBN 0-8218-4561-6.

[18] A. Bendali, A. Huard, A. Tizaoui, S. Tordeux and J.P. Vila, Asymptotic expansions of the eigenvalues of a 2-D boundaryvalue problem relative to two cavities linked by a hole of small size, C. R. Math. Acad. Sci. Paris 347(19-20) (2009), 1147-1152. doi:10.1016/j.crma.2009.09.005.

[19] G.P. Panasenko, The principle of splitting an averaged operator for a nonlinear system of equations in periodic and random skeletal structures, in: Doklady Akademii Nauk, Vol. 263, Russian Academy of Sciences, 1982, pp. 35-40.

[20] G.P. Panasenko, Averaging processes in framework structures, Mathematics of the USSR-Sbornik 50(1) (1985), 213.

[21] N.S. Bakhvalov and G. Panasenko, Homogenisation: averaging processes in periodic media: mathematical problems in the mechanics of composite materials, Vol. 36, Springer Science \& Business Media, 2012.

[22] P. Joly and S. Tordeux, Matching of asymptotic expansions for wave propagation in media with thin slots. I. The asymptotic expansion, Multiscale Model. Simul. 5(1) (2006), 304-336 (electronic). doi:10.1137/05064494X.

[23] P. Joly and A. Semin, Construction and analysis of improved Kirchoff conditions for acoustic wave propagation in a junction of thin slots, in: Paris-Sud Working Group on Modelling and Scientific Computing 2007-2008, ESAIM Proc., Vol. 25, EDP Sci., Les Ulis, 2008, pp. 44-67.

[24] A. Semin, Propagation d'ondes dans des jonctions de fentes minces, PhD thesis, Université Paris-Sud, 2010.

[25] J.C. Nédélec, Acoustic and electromagnetic equations, Applied Mathematical Sciences, Vol. 144, Springer-Verlag, New York, 2001, p. x+316, Integral representations for harmonic problems. ISBN 0-387-95155-5.

[26] W. McLean, Strongly elliptic systems and boundary integral equations, Cambridge University Press, Cambridge, 2000, p. xiv+357. ISBN 0-521-66332-6; 0-521-66375-X.

[27] E. Vasilevskaya, Open periodic waveguides. Theory and computation., PhD thesis, Université Paris 13, 2016.

[28] S. Fliss, A Dirichlet-to-Neumann approach for the exact computation of guided modes in photonic crystal waveguides, SIAM J. Sci. Comput. 35(2) (2013), B438-B461. doi:10.1137/12086697X.

[29] P. Joly, J.-R. Li and S. Fliss, Exact boundary conditions for periodic waveguides containing a local perturbation, Commun. Comput. Phys. 1(6) (2006), 945-973.

[30] S. Fliss, Etude mathématique et numérique de la propagation des ondes dans des milieux périodiques localement perturbés, $\mathrm{PhD}$ thesis, Ecole Polytechnique, 2009.

[31] D. Klindworth and K. Schmidt, An efficient calculation of photonic crystal band structures using Taylor expansions, Commun. Comput. Phys. 16(5) (2014), 1355-1388. 